\title{
THE OORT CONJECTURE ON SHIMURA CURVES IN THE TORELLI LOCUS OF CURVES
}

\author{
XIN LU AND KANG ZUO
}

\begin{abstract}
Oort has conjectured that there do not exist Shimura curves contained generically in the Torelli locus of genus- $g$ curves when $g$ is large enough. In this paper we prove the Oort conjecture for Shimura curves of Mumford type and Shimura curves parameterizing principally polarized $g$-dimensional abelian varieties isogenous to $g$-fold self-products of elliptic curves for $g>11$. We also prove that there do not exist Shimura curves contained generically in the Torelli locus of hyperelliptic curves of genus $g>7$. As a consequence, we obtain a finiteness result regarding smooth genus- $g$ curves with completely decomposable Jacobians, which is related to a question of Ekedahl and Serre.
\end{abstract}

\section{Contents}

1. Introduction

1.1. Oort's conjecture

1.2. Progress on the Oort conjecture

1.3. Main results

1.4. The main idea of proofs

1.5. Further perspectives

2. Preliminaries

2.1. Special subvarieties in Shimura varieties

2.2. Two types of Shimura curves

2.3. Logarithmic Higgs bundles on curves in $\mathcal{A}_{g}$

2.4. Families of semi-stable curves

3. Family of semi-stable curves representing a curve in $\mathcal{T}_{g}$

4. The strict Arakelov inequalities and proofs of the main results

4.1. Two types of inequalities for a family of semi-stable curves

4.2. The strict Arakelov inequalities

4.3. Proofs of the main results

5. Miyaoka-Yau type inequalities for a family of semi-stable curves

5.1. Proof of Theorems 4.1 and 4.4 based on Theorem 5.1

5.2. Proof of Theorem 5.1

6. Sharp slope inequalities fora family of semi-stable curves

6.1. Proof of Theorem 4.2

6.2. Proof of Theorem 4.3

6.3. Proof of Theorem 4.5

7. Flat part of $R^{1} \bar{f}_{*} \mathbb{Q}$ for a family of hyperelliptic semi-stable curves

Date: August 18, 2014.

2010 Mathematics Subject Classification. Primary 11G15, 14G35, 14H40; Secondary 14D07, $14 \mathrm{~K} 22$.

Key words and phrases. Shimura curves, Torelli locus, complex multiplication, Jacobians, families.

This work is supported by SFB/Transregio 45 Periods, Moduli Spaces and Arithmetic of Algebraic Varieties of the DFG (Deutsche Forschungsgemeinschaft), and partially supported by National Key Basic Research Program of China (Grant No. 2013CB834202) and NSFC. 
8. Examples and miscellaneous results

Appendix: involutions on the universal family of curves

References

\section{INTRODUCTION}

This paper is devoted to the study of the conjecture of Oort on special subvarieties of the Siegel modular variety that are contained in the Torelli locus. In this section we state the main results and explain the basic idea of the proofs.

1.1. Oort's conjecture. We start with the conjectures of Coleman and Oort. A more thorough survey of the subject is found in the beautiful paper [48.

Fix $n \geq 3$ an integer, we have $\mathcal{M}_{g}=\mathcal{M}_{g,[n]}$ the moduli space of smooth projective curves over complex number $\mathbb{C}$ of genus $g \geq 2$ with a full level $n$-structure, and $\mathcal{A}_{g}=\mathcal{A}_{g,[n]}$ the moduli space of $g$-dimensional principally polarized abelian varieties over $\mathbb{C}$ with full level- $n$ structure. In this paper we treat them as the moduli schemes over $\mathbb{C}$ of the corresponding moduli functors. No specific choice of the level $n(\geq 3)$ is made because it is only imposed to assure the representability, which plays no essential role in our study.

Recall that the Torelli morphism

$$
j^{\circ}: \mathcal{M}_{g} \longrightarrow \mathcal{A}_{g}
$$

associates to a curve its Jacobian with its canonical principal polarization and level structure. The image of $j^{\circ}$, denoted as $\mathcal{T}_{g}^{\circ}$, is a locally closed subvariety in $\mathcal{A}_{g}$, whose closure is denoted as $\mathcal{T}_{g}$. $\mathcal{T}_{g}$ is called the Torelli locus (in $\mathcal{A}_{g}$ ) and $\mathcal{T}_{g}^{\circ}$ is referred as the open Torelli locus. We also have the Torelli locus $\mathcal{T} \mathcal{H}_{g} \subseteq \mathcal{T}_{g}$ of hyperelliptic curves corresponding to Jacobians of hyperelliptic curves.

A closed subvariety $Z \subseteq \mathcal{A}_{g}$ of positive dimension is said to be contained generically in the Torelli locus (resp. the Torelli locus of hyperelliptic curves), written as $Z \Subset \mathcal{T}_{g}$ (resp. $Z \Subset \mathcal{T} \mathcal{H}_{g}$ ), if $Z \subseteq \mathcal{T}_{g}$ and $Z \cap \mathcal{T}_{g}^{\circ} \neq \emptyset$ (resp. $Z \subseteq \mathcal{T H}_{g}$ and $\left.Z \cap \mathcal{T}_{g}^{\circ} \neq \emptyset\right)$.

As is explained in [25, 40, 46, the moduli scheme $\mathcal{A}_{g}=\mathcal{A}_{g,[n]}$ is isomorphic to a connected Shimura variety, namely a geometrically connected component of the Shimura variety defined by the Shimura datum $\left(\mathrm{GSp}_{2 g}, \mathscr{H}_{g}^{ \pm}\right)$associated to the group of symplectic similitude $\mathrm{GSp}_{2 g}$, using some compact open subgroup $K(n) \subset$ $\operatorname{GSp}_{2 g}\left(\mathbb{A}_{f}\right)$, cf. Example 2.2. In $\mathcal{A}_{g}$ there are special subvarieties and totally geodesic subvarieties, the definitions of which are given later in Section 2.1, Special subvarieties are totally geodesic subvarieties containing CM points, cf. [46]. They are of particular interest because on the one hand they are locally symmetric in the sense of differential geometry, and on the other hand they parameterize abelian varieties with prescribed Hodge classes, cf. 12. Special subvarieties of dimension zero are exactly CM points, i.e. points in $\mathcal{A}_{g}$ that parameterize abelian varieties with complex multiplication.

It was conjectured by Coleman [7, that when the genus $g$ is sufficiently large, there should be at most finitely many $\mathrm{CM}$ points on $\mathcal{A}_{g}$ contained in the open Torelli locus $\mathcal{T}_{g}^{\circ}$. Oort [55] made the following conjecture by combining Coleman's idea with the conjecture of André-Oort:

Conjecture 1.1 (Oort). For g large, there does not exist a special subvariety of positive dimension contained generically in the Torelli locus $\mathcal{T}_{g}$.

The André-Oort conjecture predicts that in a Shimura variety, a closed geometrically irreducible subvariety is special if and only if it contains a Zariski dense subset 
of $\mathrm{CM}$ points. It is thus immediate that the formulation of Oort is equivalent to the one by Coleman modulo André-Oort. The readers are referred to [54], 63], [71, etc. for surveys on the recent progress towards the André-Oort conjecture.

1.2. Progress on the Oort conjecture. Although Coleman made his conjecture for $g \geq 4$, counterexamples have been found for $4 \leq g \leq 7$. However, if one aims at the non-existence of special subvarieties of a certain type with $g$ sufficiently large, then much more evidence is available, as the concrete "type" of the special subvarieties often imposes constraints so that no universal family of curves could be produced from such special subvarieties subject to the Torelli morphism (cf. [5. 48]).

In 21, based on the properties of the mapping class groups (cf. [17]), Hain proved, under some natural technical assumptions, that a special subvariety $Z$ of $\mathcal{A}_{g}$ should be either a ball quotient or that its intersection with the Torelli locus of hyperelliptic curves $\mathcal{T} \mathcal{H}_{g}$ should be a divisor in $Z$. This suggests that the ball quotients should play a special role in the study of the conjecture. Building on Hain's method, de Jong and Zhang [27] have proved that $Z$ cannot be a Hecke translate of a Hilbert modular subvariety if $g \geq 5$, which has also been proved for $g=4$ by Bainbridge and Möller [1] using degeneration techniques and independent of properties of the mapping class groups.

In [26], de Jong and Noot have proposed an approach based on an $p$-adic obstruction constructed by Dwork-Ogus [14] and they proved that base varieties of some specific universal families of curves arising from cyclic covers of $\mathbb{P}^{1}$ are not contained generically in $\mathcal{T}_{g}$. Recently, Moonen 47. has extended de Jong and Noot's result and proved that there are exactly twenty families of curves coming from cyclic covers of $\mathbb{P}^{1}$ such that the base varieties are contained generically in $\mathcal{T}_{g}$, which implies that Conjecture 1.1 holds if the corresponding special subvarieties arise from a universal cyclic cover of $\mathbb{P}^{1}$.

In 34, Kukulies proved Oort's conjecture for rational Shimura curves parameterizing principally polarized abelian varieties isogenous to $g$-fold self-product of elliptic curves for $g \gg 0$. His approach combines ideas of Möller, Viehweg and the second named author on the characterization of Shimura curves and the Sato-Tate conjecture for modular curves, which is of arithmetic nature.

There has been also other progress on the conjecture, cf. [8, 18, 20, 42, 44, 57, 61] and further discussions in [48].

1.3. Main results. In this paper we focus on the conjecture of Oort for Shimura curves of the following types::

I. Shimura curves parameterizing principally polarized $g$-dimensional abelian varieties that are isogenous to a $g$-fold self-product of some elliptic curve;

II. Shimura curves of Mumford type;

III. Shimura curves in the Torelli locus of hyperelliptic curves.

In this paper Shimura curves are special subvarieties of dimension one, cf. Remark 2.8. The precise definition of the Shimura curves of type I and II are given in Section 2.2. While Shimura curves of type I are of PEL type, Shimura curves of Mumford type constructed in 69 by generalizing Mumford's original example in [51, are not of PEL-type except precisely two classes (see Section 2.2).

Our first main result is the following:

Theorem A. For $g>11$, there does not exist a Shimura curve of types I or II contained generically in $\mathcal{T}_{g}$.

This answers a question of Moonen and Oort [48, Question 6.7] in the onedimensional case. The result by Kukulies [34] also studies Shimura curves of type I, 
but it is more restrictive: it only deals with rational Shimura curves, and the bound for $g$ depends on the curve in question, which is not uniform. Our approach is of differential-geometric nature. It gives an explicit lower bound, without resorting to deep number-theoretic results like the Sato-Tate conjecture.

Note that Theorem A also implies a partial answer to a question raised by Ekedahl and Serre [15, where they asked for the existence of a smooth curve of higher genus whose Jacobian is completely decomposable, i.e., isogenous to a $g$-fold product of elliptic curves. In fact we obtain the following corollary of finiteness:

Corollary B. For each fixed integer $g>11$, there exist, up to isomorphism, at most finitely many smooth projective curves of genus $g$ whose Jacobians are isogenous to $g$-fold self-product of a single elliptic curve with bounded isogenous degrees.

In 27. De Jong and Zhang have shown that Hecke translates of Hilbert modular varieties associated to totally real fields of degree $g$ over $\mathbb{Q}$ are not contained generically in the Torelli locus $\mathcal{T}_{g}$ for $g \geq 5$ (which also holds for $g=4$ proved by Bainbridge-Möller [1]). Using the non-existence of Shimura curves of type I in the Torelli locus, we obtain an alternative approach to their result, under a slightly varied formulation with $g>11$ :

Corollary C (real multiplication). Let $F$ be a totally real étale $\mathbb{Q}$-algebra of dimension $g$ over $\mathbb{Q}$, and let $Z \subseteq \mathcal{A}_{g}$ be a Hecke translate of the generalized Hilbert modular subvariety defined by $\left(\mathbf{G}^{F}, X^{F}\right)$ (cf. Definition 2.11). Then $Z$ is not contained generically in $\mathcal{T}_{g}$ for $g>11$.

Here by totally real étale $\mathbb{Q}$-algebra we mean a product of finitely many totally real number fields, and the generalized Hilbert modular subvariety they define are, up to finite covering, products of usual Hilbert modular varieties (involving several totally real fields). Such Shimura data contain the subdatum $\left(\mathrm{GL}_{2}, \mathscr{H}_{1}^{ \pm}\right)$induced by the natural embedding $\mathbb{Q} \hookrightarrow F$, hence the special subvariety $Z$ they define contain a Shimura curve of type I. Note that the idea of 27] goes back to [21] which relies on properties of the mapping class groups studied in [17. It only works for the usual Hilbert modular varieties associated to a totally real field of dimension $g \geq 5$. It does not cover the case of the $g$-fold product of modular curves embedded in $\mathcal{A}_{g}$, because in this case the lattice involved is essentially reducible with low rank factors, i.e. commensurable with $S L_{2}(\mathbb{Z})^{g}$. Our approach focuses on modular curves diagonally embedded in these generalized Hilbert modular varieties, which has no restriction on the dimension of the factor fields of the totally real étale algebra, and the proof does not involve the mapping class groups.

The following is a weaker result regarding Oort's conjecture for Shimura curves of types I and II, already proved in our previous preprint [38, Theorem 1.2].

Theorem D. For $g>4$, there does not exist any one-dimensional family of semistable curves of genus $g$ with strictly maximal Higgs field.

Higgs bundles and Higgs fields on curves will be among the main tools in our proofs. They are briefly recalled in the next subsection, and more details will be given in Sections 2.3 and 3. For a semi-stable family of curves with strictly maximal Higgs field, its image under the Torelli morphism is already a Shimura curve in $\mathcal{A}_{g}$ due to [69, and hence Theorem $\mathrm{A}$ implies Theorem $\mathrm{D}$ for $g>11$. On the other hand, the converse is not true, namely not all Shimura curves of type I or II contained generically in $\mathcal{T}_{g}$ arise this way; see Section 3 for further discussions, and especially Example 8.3 for $g=3$.

Our last main result focuses on Shimura curves in the Torelli locus of hyperelliptic curves, which holds more generally for totally geodesic curves in $\mathcal{A}_{g}$ : 
Theorem E. For $g>7$, there does not exist totally geodesic curves contained generically in the Torelli locus of hyperelliptic curves $\mathcal{T} \mathcal{H}_{g}$.

1.4. The main idea of proofs. In this subsection we explain the main idea of the proofs.

As is mentioned earlier, we intend to exclude the existence of Shimura curves of certain type in the open Torelli locus using the natural constraints from the geometry of special subvarieties. For the three types of Shimura curves studied in this paper, the constraints mainly come from two types of inequalities:

(i). Arakelov inequalities of Higgs bundles for curves in $\mathcal{A}_{g}$, which are actually equalities when applied to Shimura curves;

(ii). strict Arakelov inequalities of Higgs bundles for curves contained generically in the Torelli locus.

The Arakelov (in)equalities of Higgs bundles play a crucial role in the works 45. 69 characterizing the geometry of totally geodesic subvarieties in Shimura varieties. The survey [67] by Viehweg is highly recommended.

In our case, the universal family of abelian varieties $\mathfrak{h}: \mathcal{X}_{g} \rightarrow \mathcal{A}_{g}$ gives rise to the Higgs bundle $(E, \theta)$ on $\mathcal{A}_{g}$, where $E=E^{1,0} \oplus E^{0,1}$ is the graded quotient of the Hodge filtration on the $\mathbb{Q}$-VHS whose underlying local system is $\mathbb{V}=R^{1} \mathfrak{h}_{*} \mathbb{Q}_{\mathcal{X}_{g}}$, and $\theta$ is induced by the Gauss-Manin connection. For a smooth closed curve $\phi: C \hookrightarrow \mathcal{A}_{g}$ with a suitable smooth compactification $\bar{C}$ by joining a finite set of cusps $\Delta_{\bar{C}}$, by pulling-back along $\phi$ one has the universal family of abelian varieties $h: X \rightarrow C$ and a local system $\mathbb{V}_{C}:=R h_{*} \mathbb{Q}_{X}=\phi^{*} \mathbb{V}$, which underlies a $\mathbb{Q}$-VHS whose associated Higgs bundle on $C$ extends to a logarithmic Higgs bundle $\left(E_{\bar{C}}, \theta_{\bar{C}}\right)$ on $\bar{C}$. $\left(E_{\bar{C}}, \theta_{\bar{C}}\right)$ decomposes further into a direct sum of Higgs bundles (cf. [19] or [31]):

$$
\left(E_{\bar{C}}^{1,0} \oplus E_{\bar{C}}^{0,1}, \theta_{\bar{C}}\right)=\left(A_{\bar{C}}^{1,0} \oplus A_{\bar{C}}^{0,1},\left.\theta_{\bar{C}}\right|_{A_{\bar{C}}^{1,0}}\right) \oplus\left(F_{\bar{C}}^{1,0} \oplus F_{\bar{C}}^{0,1}, 0\right),
$$

where $A_{\bar{C}}^{1,0}$ is an ample vector bundle, while $F_{\bar{C}}^{1,0}$ and $F_{\bar{C}}^{0,1}$ are flat vector bundles associated to unitary local subsystems $\mathbb{F}_{C}^{1,0} \oplus \mathbb{F}_{C}^{0,1} \subseteq \mathbb{V}_{C}$.

Definition $1.2(\underline{68})$. The Higgs bundle $\left(E_{\bar{C}}^{1,0} \oplus E_{\bar{C}}^{0,1}, \theta_{\bar{C}}\right)$ is said to be with maximal Higgs field if

$$
\left.\theta_{\bar{C}}\right|_{A_{\bar{C}}^{1,0}}: A_{\bar{C}}^{1,0} \longrightarrow A_{\bar{C}}^{0,1} \otimes \Omega_{\bar{C}}^{1}\left(\log \Delta_{\bar{C}}\right)
$$

is an isomorphism, and to be with strictly maximal Higgs field if furthermore $F_{\bar{C}}^{1,0} \oplus$ $F_{\bar{C}}^{0,1}=0$. By $\left[68\right.$ the Higgs field $\theta_{\bar{C}}$ is strictly maximal (resp. maximal) if and only if the following Arakelov equality holds

$$
\begin{aligned}
& \operatorname{deg} E_{\bar{C}}^{1,0}=\frac{g}{2} \cdot \operatorname{deg} \Omega_{\bar{C}}\left(\log \Delta_{\bar{C}}\right), \\
&\left(\text { resp. } \quad \operatorname{deg} E_{\bar{C}}^{1,0}=\frac{\operatorname{rank} A_{\bar{C}}^{1,0}}{2} \cdot \operatorname{deg} \Omega_{\bar{C}}^{1}\left(\log \Delta_{\bar{C}}\right)\right) .
\end{aligned}
$$

The following theorem gives numerical characterizations of Shimura curves, and more generally of totally geodesic curves:

Theorem $1.3([44,69])$. Let $C \subseteq \mathcal{A}_{g}$ be a smooth closed curve, and $\left(E_{\bar{C}}^{1,0} \oplus E_{\bar{C}}^{0,1}, \theta_{\bar{C}}\right)$ the associated logarithmic Higgs bundle. Then

(i). $C$ is a Shimura curve of type I or II if and only if the associated logarithmic Higgs bundle $\left(E_{\bar{C}}^{1,0} \oplus E_{\bar{C}}^{0,1}, \theta_{\bar{C}}\right)$ has the strictly maximal Higgs field.

(ii). $C$ is a totally geodesic curve if and only if the associated logarithmic Higgs bundle has maximal Higgs field. 
Now Theorems $\mathrm{A}$ and $\mathrm{E}$ are immediate consequences of the theorem above and the following strict Arakelov inequalities for a smooth closed curve $C \Subset \mathcal{T}_{g} \subseteq \mathcal{A}_{g}$.

Theorem 1.4. Let $C \subseteq \mathcal{A}_{g}$ be a smooth closed curve, and $\left(E_{\bar{C}}^{1,0} \oplus E_{\bar{C}}^{0,1}, \theta_{\bar{C}}\right)$ be the associated logarithmic Higgs bundle.

(i). If $C \Subset \mathcal{T}_{g}$ with $g>11$, then

$$
\operatorname{deg} E_{\bar{C}}^{1,0}<\frac{g}{2} \cdot \operatorname{deg} \Omega_{\bar{C}}^{1}\left(\log \Delta_{\bar{C}}\right) .
$$

(ii). If $C \Subset \mathcal{T} \mathcal{H}_{g}$ with $g>7$, then

$$
\operatorname{deg} E_{\bar{C}}^{1,0}<\frac{\operatorname{rank} A_{\bar{C}}^{1,0}}{2} \cdot \operatorname{deg} \Omega \frac{1}{C}\left(\log \Delta_{\bar{C}}\right) .
$$

Details of the proof of the strict Arakelov inequalities are given in Section 4.2 . Roughly speaking, we have the following diagram in which the central square is Cartesian:

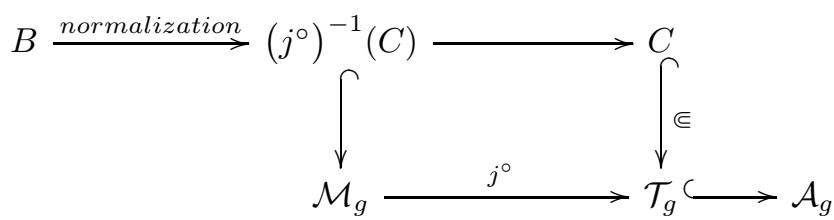

By pulling back the universal family over $\mathcal{M}_{g}$ to $B$, one obtains a family $f: S \rightarrow B$ of curves, which can be naturally extended to a family $\bar{f}: \bar{S} \rightarrow \bar{B}$ of semi-stable curves over the smooth compactification $\bar{B} \supseteq B$. Then Theorem 1.4 will be a combination of two types of inequalities (Miyaoka-Yau type inequality and sharp slope inequality) for a family of semi-stable curves, the proofs of which rely highly on the geometrical property of fibred surfaces.

1.5. Further perspectives. The method of the paper was stimulated by the numerical characterization of Shimura curves using the Arakelov equality given by Möller, Viehweg and the second named author [44, 69. In 45], they generalized it to high dimension, and obtained the numerical characterization of special subvarieties of arbitrary dimension in $\mathcal{A}_{g}$, where the Arakelov equality plays a key role. It suggests a new way to prove Oort's conjecture by proving a strict Arakelov inequality for subvarieties of $\mathcal{A}_{g}$ which are contained generically in $\mathcal{T}_{g}$ for $g \gg 0$.

In 21, Hain has dealt with those special subvarieties in $\mathcal{T}_{g}$ containing no divisor which is also special. It does not treat special subvarieties associated to groups like $S O(n, 2)$ and $S U(n, 1)$, which are of real rank one. However, in such special subvarieties, one can construct special curves following [32, 33. This is the subject of our ongoing research, where we intend to exclude such special subvarieties by studying the strict Arakelov inequalities for special curves in them, cf. [?].

The paper is organized as follows. In Section 2 we collect facts about special subvarieties in Shimura varieties, logarithmic Higgs bundles, and surfaces fibred over curves with semi-stable fibers. In Section 3 we construct a family $\bar{f}: \bar{S} \rightarrow \bar{B}$ of semi-stable curves representing a smooth closed curve $C \Subset \mathcal{T}_{g}$, and we explain the relation between the associated logarithmic Higgs bundles over $\bar{B}$ and over $\bar{C}$. In Section 4. We prove the strict Arakelov inequalities and the main results, based on two types of inequalities (Miyaoka-Yau type inequality and sharp slope inequality) for a family of semi-stable curves, whose proofs are given in Sections 5 and 6 respectively. An intermediate result needed in Section 4 is proved in Section 7. which focuses on the flat part of the Higgs bundle associated to a family of semistable hyperelliptic curves. Finally in Section 8, we present examples of Shimura curves contained generically in the Torelli locus. 


\section{Preliminaries}

2.1. Special subvarieties in Shimura varieties. We first recall the general notions of Shimura data and Shimura varieties, following [11] and [40].

Definition 2.1 (Shimura data and Shimura varieties). Write $\mathbb{S}=\operatorname{Res}_{\mathbb{C} / \mathbb{R}} \mathbb{G}_{\mathrm{m}}$ for the Deligne torus.

(1) A Shimura datum is a pair $(\mathbf{G}, X)$ where

SD1. $\mathbf{G}$ is a connected reductive $\mathbb{Q}$-group, such that $\mathbf{G}^{\text {ad }}$ has no compact factors defined over $\mathbb{Q}$;

SD2. $X$ is a $\mathbf{G}(\mathbb{R})$-conjugacy class of homomorphisms of $\mathbb{R}$-groups $x: \mathbb{S} \rightarrow \mathbf{G}_{\mathbb{R}}$, such that

- the composition Ad $\circ x: \mathbb{S} \rightarrow \mathbf{G}_{\mathbb{R}} \rightarrow \mathrm{GL}_{\mathbb{R}}\left(\mathfrak{g}_{\mathbb{R}}\right)$ defines a pure Hodge structure of type $\{(-1,1),(0,0),(1,-1)\}$ on the Lie algebra $\mathfrak{g}=$ Lie $\mathbf{G}$;

- the conjugation by $x(\sqrt{-1})$ induces a Cartan involution on $\mathbf{G}^{\text {ad }}(\mathbb{R})$.

It turns out that each connected component of $X$ is an Hermitian symmetric domain. We write $\mathbf{G}(\mathbb{R})_{+}$for the stabilizer in $\mathbf{G}(\mathbb{R})$ of any such connected component, and we put $\mathbf{G}(\mathbb{Q})_{+}=\mathbf{G}(\mathbb{R})_{+} \cap \mathbf{G}(\mathbb{Q})$.

(2) Let $(\mathbf{G}, X)$ be a Shimura datum and let $K \subseteq \mathbf{G}\left(\mathbb{A}_{f}\right)$ be a compact open subgroup. The Shimura variety associated to $(\mathbf{G}, X)$ at level $K$ is an algebraic variety $M_{K}(\mathbf{G}, X)$ whose $\mathbb{C}$-points are described by the formula

$$
M_{K}(\mathbf{G}, X)(\mathbb{C})=\mathbf{G}(\mathbb{Q}) \backslash\left[X \times \mathbf{G}\left(\mathbb{A}_{f}\right) / K\right],
$$

where $\mathbf{G}(\mathbb{Q})$ acts on $X \times \mathbf{G}\left(\mathbb{A}_{f}\right) / K$ diagonally. Fix $X^{+}$a connected component of $X$, we actually have the following expression of geometrically connected components

$$
M_{K}(\mathbb{C})=\coprod_{a} \Gamma_{K}(a) \backslash X^{+},
$$

where $a$ runs through a set of representatives of the quotient $\mathbf{G}(\mathbb{Q})_{+} \backslash \mathbf{G}\left(\mathbb{A}_{f}\right) / K$, and $\Gamma_{K}(a)=\mathbf{G}(\mathbb{Q})_{+} \cap a K a^{-1}$ acts on $X^{+}$through $\mathbf{G}^{\text {ad }}(\mathbb{R})^{+}$.

By [2], each $\Gamma_{K}(a) \backslash X^{+}$is a quasi-projective algebraic variety over $\mathbb{C}$. Moreover $M_{K}(\mathbf{G}, X)$ admits a canonical model over some number fields (cf. [39]).

Example 2.2 (Siegel modular variety, cf. [40, $\S 6])$. Let $\left(V=\mathbb{Q}^{2 g}, \psi\right)$ be the standard symplectic space of dimension $2 g$, with symplectic basis $e_{1}, e_{-1}, \cdots, e_{g}, e_{-g}$ such that $\psi\left(e_{i}, e_{-j}\right)=\delta_{i j}$. Denote by $\mathrm{GSp}_{2 g}$ the connected reductive $\mathbb{Q}$-group of symplectic similitude of $(V, \psi)$, and $\mathscr{H}_{g}^{ \pm}$be the Siegel double half space of complex symmetric $g \times g$ matrices with definite imaginary part. Then $\left(\mathrm{GSp}_{2 g}, \mathscr{H}_{g}^{ \pm}\right)$ is a Shimura datum, because $\mathscr{H}_{g}^{ \pm}$is identified with the set of homomorphisms $h: \mathbb{S} \rightarrow \mathrm{GSp}_{2 g, \mathbb{R}}$ such that the composition $\mathbb{S} \rightarrow \mathrm{GSp}_{2 g, \mathbb{R}} \rightarrow \mathrm{GL}_{2 g, \mathbb{R}}$ defines a complex structure on $V_{\mathbb{R}}$ with $(x, y) \mapsto \psi(x, h(\sqrt{-1}) y)$ symmetric definite.

Let $n \geq 3$ be an integer and take $K$ to be the principal $n$-th congruence subgroup

$$
K=K(n)=\operatorname{Ker}\left(\operatorname{GSp}_{2 g}(\hat{\mathbb{Z}}) \rightarrow \operatorname{GSp}_{2 g}(\mathbb{Z} / n)\right) .
$$

Then $M_{K}\left(\mathrm{GSp}_{2 g}, \mathscr{H}_{g}^{ \pm}\right)$is just $\mathcal{A}_{g,[n]}$, the moduli scheme over $\mathbb{Q}$ parameterizing principally polarized abelian varieties with full level- $n$ structure. The set of geometrically connected components of $M_{K}\left(\mathrm{GSp}_{2 g}, \mathscr{H}_{g}^{ \pm}\right)$is in bijection with the set $\mu_{n}$ of $n$-th roots of 1 , each of them isomorphic to $\Gamma(n) \backslash \mathscr{H}_{g}^{+}$, with $\Gamma(n)=\operatorname{Ker}\left(\operatorname{Sp}_{2 g}(\mathbb{Z}) \rightarrow\right.$ $\left.\mathrm{Sp}_{2 g}(\mathbb{Z} / n)\right)$ the $n$-th principal congruence subgroup.

For simplicity we will use $\mathcal{A}_{g}$ to denote the fixed connected component indexed by $1 \in \mu_{n}$ of the Shimura variety $M_{K}\left(\mathrm{GSp}_{2 g}, \mathscr{H}_{g}^{ \pm}\right)$described above. $\mathcal{A}_{g}$ has its canonical model over $\mathbb{Q}\left(\zeta_{n}\right)$ the $n$-th cyclotomic field. It is isomorphic to the 
moduli scheme $\mathcal{A}_{g, 1, n}$ constructed by Mumford in [50. The whole Shimura variety $M_{K}\left(\mathrm{GSp}_{2 g}, \mathscr{H}_{g}^{ \pm}\right)$is the $\mathbb{Q}$-scheme by composing $\mathcal{A}_{g, 1, n} \rightarrow \operatorname{Spec} \mathbb{Q}\left(\zeta_{n}\right)$ with $\operatorname{Spec} \mathbb{Q}\left(\zeta_{n}\right) \rightarrow \operatorname{Spec} \mathbb{Q}$, cf. [25, Chapter 6, Section 6.4].

We will mainly focus on geometrically connected components:

Definition 2.3 (connected Shimura data and varieties). (1) A connected Shimura datum is a triple $\left(\mathbf{G}, X ; X^{+}\right)$where $(\mathbf{G}, X)$ is a Shimura datum and $X^{+}$is a connected component of $X$.

(2) A connected Shimura variety is a quotient space of the form $Z=\Gamma \backslash X^{+}$, where $X^{+}$comes from some connected Shimura datum $\left(\mathbf{G}, X ; X^{+}\right)$, and $\Gamma$ is a congruence subgroup of $\mathbf{G}^{\mathrm{der}}(\mathbb{R})_{+}:=\mathbf{G}(\mathbb{R})_{+} \cap \mathbf{G}^{\mathrm{der}}(\mathbb{R})$, namely the stabilizer of $X^{+}$in $\mathbf{G}^{\text {der }}(\mathbb{R})$.

Remarks 2.4. (i). The definition above of connected Shimura varieties differs slightly from the one adopted in 40 , where $\Gamma$ is taken to be a congruence subgroup of $\mathbf{G}^{\text {ad }}(\mathbb{Q})^{+}$. Since the center of $\mathbf{G}(\mathbb{R})_{+}$acts on $X^{+}$trivially, it only differs from our version of connected Shimura varieties by a finite covering.

(ii). Just like general Shimura varieties in the sense of Definition 2.1 connected Shimura varieties admit canonical models over suitable number fields. In this paper it suffices to treat them as complex algebraic varieties.

Definition 2.5 (special subvarieties). Let $\left(\mathbf{G}, X ; X^{+}\right)$be a connected Shimura datum, which defines a connected Shimura variety $Z=\Gamma \backslash X^{+}$.

(1) A Shimura subdatum of $(\mathbf{G}, X)$ is a Shimura datum $\left(\mathbf{G}^{\prime}, X^{\prime}\right)$ such that $\mathbf{G}^{\prime}$ is a $\mathbb{Q}$-subgroup of $\mathbf{G}$ and $X^{\prime}$ is the $\mathbf{G}^{\prime}(\mathbb{R})$-orbit of some $h \in X$ such that $h(\mathbb{S}) \subseteq \mathbf{G}_{\mathbb{R}}^{\prime}$. Note that $X^{\prime} \hookrightarrow X$ is equivariant with respect to $\mathbf{G}^{\prime}(\mathbb{R}) \hookrightarrow \mathbf{G}(\mathbb{R})$.

A connected Shimura subdatum of $\left(\mathbf{G}, X ; X^{+}\right)$is a connected Shimura datum $\left(\mathbf{G}^{\prime}, X^{\prime} ; X^{\prime+}\right)$, such that $\left(\mathbf{G}^{\prime}, X^{\prime}\right)$ is a Shimura subdatum of $(\mathbf{G}, X)$ and $X^{\prime+}$ is a connected component of $X^{\prime}$ which is contained in $X^{+}$.

(2) Write $\mathbf{u}_{\Gamma}$ for canonical projection $X^{+} \rightarrow \Gamma \backslash X^{+}, x \mapsto \Gamma x$, which we call the uniformization map of $Z$. Then a special subvariety of $Z$ is of the form $Z^{\prime}=$ $\mathbf{u}_{\Gamma}\left(X^{\prime+}\right)$, where $X^{\prime+}$ comes from some subdatum $\left(\mathbf{G}^{\prime}, X^{\prime} ; X^{\prime+}\right)$.

$Z^{\prime}$ is actually the image of a morphism between connected Shimura varieties $\Gamma^{\prime} \backslash X^{\prime+} \rightarrow \Gamma \backslash X$ for some congruence subgroup $\Gamma^{\prime} \subseteq \mathbf{G}^{\prime \text { der }}(\mathbb{Q})_{+}$, and it is a closed subvariety of $Z$ over $\mathbb{C}$, which actually admits a model over some number field.

We mention briefly the notion of Hecke translation in the setting of connected Shimura varieties.

Definition 2.6 (Hecke translation). Let $Z=\Gamma \backslash X^{+}$be a connected Shimura variety defined by $\left(\mathbf{G}, X ; X^{+}\right)$. For $a \in \mathbf{G}(\mathbb{Q})_{+}$, the Hecke correspondence associated to $a$ is the following diagram

$$
\Gamma \backslash \stackrel{q}{\leftarrow} \Gamma_{a} \backslash X^{+} \stackrel{q_{a}}{\rightarrow} \Gamma \backslash X^{+}
$$

where

- $\Gamma_{a}=\Gamma \cap a^{-1} \Gamma a$;

- $q\left(\Gamma_{a} x\right)=\Gamma x$ and $q_{a}\left(\Gamma_{a} x\right)=\Gamma a x$.

Both $q$ and $q_{a}$ are finite morphisms of degree equal to $\left[\Gamma: \Gamma_{a}\right]$. For $Z^{\prime}$ a closed irreducible subvariety of $Z$, any irreducible component of $q_{a}\left(q^{-1} Z^{\prime}\right)$ is called a Hecke translate of $Z^{\prime}$ by $a$.

This also makes sense for general cycles in $Z$, where we write $q_{a *} q^{*}\left(Z^{\prime}\right)$ as multiplicities could arise, and the map $q_{a *} q^{*}$ is called the Hecke operator associated to $a$ (acting on the space of cycles). 
Remark 2.7. It is easy to verify that the Hecke translate of a special subvariety remains special. Moreover, for any special point $s \in Z$, the union of all the Hecke translates of $s$ using $a \in \mathbf{G}(\mathbb{Q})_{+}$is dense in $Z$ for the analytic topology, because $\mathbf{G}(\mathbb{Q})_{+}$is dense in $\mathbf{G}(\mathbb{R})_{+}$by the real approximation of linear $\mathbb{Q}$-groups.

Remark 2.8 (terminology). In [66] etc. a Shimura subvariety of $M_{K}(\mathbf{G}, X)$ is the image of a morphism between Shimura varieties $f: M_{K^{\prime}}\left(\mathbf{G}^{\prime}, X^{\prime}\right) \rightarrow M_{K}(\mathbf{G}, X)$ given by a morphism of Shimura data $f:\left(\mathbf{G}^{\prime}, X^{\prime}\right) \rightarrow(\mathbf{G}, X)$, using some compact open subgroup $K^{\prime} \subset \mathbf{G}^{\prime}\left(\mathbb{A}_{f}\right) \cap K$. In this setting we have the notion of Hecke correspondence given by adelic points $a \in \mathbf{G}\left(\mathbb{A}_{f}\right)$, and special subvarieties are defined as geometrically irreducible components of the (adelic) Hecke translate of a Shimura subvariety.

It turns out that the special subvarieties thus defined are subvarieties contained in suitable connected components of $M_{K}(\mathbf{G}, X)$. Shifting between connected components following [6. Lemma 2.13], we see that the special subvarieties in the sense of [66] are the same as ours when restricted to a connected component of $M_{K}(\mathbf{G}, X)$. In particular, the notion of Hecke translation is not involved in our definition of special subvarieties, although it will be needed elsewhere, like the description of Shimura curves of type I.

Since we only work with connected Shimura varieties, the adjective "connected" will be often omitted if no ambiguity occurs, and our special subvarieties will be also called Shimura subvarieties, like the Shimura curves in Section 2.2

To end the subsection, we include the notion of totally geodesic subvarieties following 46]:

Definition 2.9 (totally geodesic subvarieties). Let $Z=\Gamma \backslash X^{+}$be a Shimura variety defined by $\left(\mathbf{G}, X ; X^{+}\right)$, with $\mathbf{u}_{\Gamma}$ the uniformization map.

A totally geodesic subvariety of $Z$ is of the form $\mathbf{u}_{\Gamma}\left(Y_{1}^{+} \times\left\{y_{2}\right\}\right)$, where for some subdatum $\left(\mathbf{H}, Y ; Y^{+}\right) \subseteq\left(\mathbf{G}, X ; X^{+}\right)$we have $\left(\mathbf{H}^{\text {ad }}, Y^{\text {ad }} ; Y^{\text {ad+ }}\right) \simeq\left(\mathbf{H}_{1}, Y_{1} ; Y_{1}^{+}\right) \times$ $\left(\mathbf{H}_{2}, Y_{2} ; Y_{2}^{+}\right)$and $y_{2} \in Y_{2}^{+}$. Here $\left(\mathbf{H}^{\text {ad }}, Y^{\text {ad }} ; Y^{\text {ad }+}\right)$ is deduced from $\left(\mathbf{H}, Y ; Y^{+}\right)$by taking $Y^{\text {ad }}$ to be the $\mathbf{H}^{\text {ad }}(\mathbb{R})$-orbit of the composition $\mathbb{S} \stackrel{y}{\rightarrow} \mathbf{H}_{\mathbb{R}} \rightarrow \mathbf{H}_{\mathbb{R}}^{\text {ad }}$ using any $y \in Y$; in particular, $Y^{+}=Y^{\text {ad+ }}$ as the center of $\mathbf{H}(\mathbb{R})$ acts on $Y$ trivially.

Remark 2.10. Totally geodesic subvarieties can also be defined in terms of differential geometry. However, we do not need the fine geometry of these subvarieties, except for a numerical characterization in the case of curves due to Viehweg and the second named author, cf. Theorem 1.3 and the original paper [69.

2.2. Two types of Shimura curves. We recall briefly the definition of two types of Shimura curves that will be studied later.

The first class of Shimura curves are modular curves "diagonally" embedded in $\mathcal{A}_{g}$, and the embedding factors through a slightly generalized form of Hilbert modular varieties, which we describe as follows

Definition 2.11 (Hilbert modular variety). (1) A totally real étale $\mathbb{Q}$-algebra is a finite dimensional étale $\mathbb{Q}$-algebra $F$ (necessarily commutative) such that the $\mathbb{R}$ algebra $F \otimes_{\mathbb{Q}} \mathbb{R}$ is isomorphic to the direct product $\mathbb{R}$-algebra $\mathbb{R}^{g}$, with $g=\operatorname{dim}_{\mathbb{Q}} F$. It is clear that $F$ is isomorphic to a finite product of totally real number fields $\prod_{i} F_{i}$ with $\sum_{i}\left[F_{i}: \mathbb{Q}\right]=g$, and $F$ is a $\mathbb{Q}$-form of the product algebra $\mathbb{Q}^{g}$.

(2) Let $F$ be a totally real étale $\mathbb{Q}$-algebra of dimension $g$. Then $\mathbf{G}:=\operatorname{Res}_{F / \mathbb{Q}} \mathrm{GL}_{2}$ is a $\mathbb{Q}$-form of the $d$-fold product $\mathrm{GL}_{2}^{d}$, which splits after the base change $\mathbb{Q} \rightarrow \mathbb{R}$. We thus have $\mathbf{G}_{\mathbb{R}} \simeq \prod_{\sigma} \mathrm{GL}_{2, \sigma}$, where $\mathrm{GL}_{2, \sigma}$ stands for $\mathrm{GL}_{2, \mathbb{R}}$ indexed by one of the $g$ distinct homomorphisms of $\mathbb{R}$-algebras $\sigma: F \otimes \mathbb{Q} \rightarrow \mathbb{R}$. 
Let $X$ be the $\mathbf{G}(\mathbb{R})$-conjugacy class of the homomorphism

$$
h: \mathbb{S} \rightarrow\left(\operatorname{Res}_{F / \mathbb{Q}} \mathrm{GL}_{2}\right)_{\mathbb{R}}, \quad \rho \exp (\sqrt{-1} \theta) \mapsto\left(\left[\begin{array}{cc}
\rho \cos \theta & \rho \sin \theta \\
-\rho \sin \theta & \rho \cos \theta
\end{array}\right]\right)_{\sigma}
$$

using $\mathbf{G}_{\mathbb{R}} \simeq \prod_{\sigma} \mathrm{GL}_{2, \sigma}$. It is then immediate that $(\mathbf{G}, X)$ is a Shimura datum in the sense of [11, with $X \simeq \prod_{\sigma} \mathscr{H}_{1, \sigma}^{ \pm}$the $g$-fold product of $\mathscr{H}_{1}^{ \pm}$.

Note that the center of $\mathbf{G}$ is $\operatorname{Res}_{F / \mathbb{Q}} \mathbb{G}_{\mathrm{m}}$, which is too big to be put into $\mathrm{GSp}_{2 g}$ directly respecting the moduli interpretation. We thus restrict to the $\mathbb{Q}$-subgroup

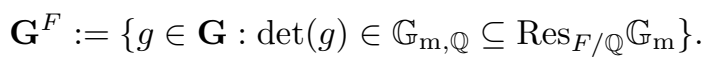

In other words, we may start with an embedding $\operatorname{Res}_{F / \mathbb{Q}} \mathrm{SL}_{2} \hookrightarrow \mathrm{Sp}_{2 g}$. This can be done by choosing an $F$-linear structure on the $\mathbb{Q}$-Lagrangian decomposition $V=V_{+} \oplus V_{-}$described in Example 2.2, say identify $e_{1}, \cdots, e_{g}$ with a $\mathbb{Q}$-basis of $F$ and extend this $F$-linear structure from $V_{+}=\bigoplus_{i=1}^{g} \mathbb{Q} e_{i}$ to $V_{-}=\bigoplus_{i=1}^{g} \mathbb{Q} e_{-i}$ respecting the symplectic form $\psi$. The embedding $\operatorname{Res}_{F / \mathbb{Q}} \mathrm{SL}_{2} \hookrightarrow \mathrm{Sp}_{2 g}$ extends to $\mathbf{G}^{F} \hookrightarrow \mathrm{GSp}_{2 g}$ by joining a central $\mathbb{Q}$-torus isomorphic to $\mathbb{G}_{\mathrm{m}}$. It is then clear that the homomorphism $h$ mentioned above has its image in $\mathbf{G}_{\mathbb{R}}^{F}$, and we get a smaller Shimura datum $\left(\mathbf{G}^{F}, X^{F}=\mathbf{G}^{F}(\mathbb{R}) \cdot h\right)$, which is a subdatum of $\left(\mathrm{GSp}_{2 g}, \mathscr{H}_{g}^{ \pm}\right)$.

The moduli interpretation of Shimura subvarieties associated to $\left(\mathbf{G}^{F}, X^{F}\right)$ is similar to the case of usual Hilbert modular varieties, namely they classify abelian varieties with endomorphism by $F$ up to isogeny (plus suitable level structures and polarization constraints).

Definition 2.12 (Shimura curves of type I). In Shimura subvarieties of $\mathcal{A}_{g}$ defined by the subdatum $\left(\mathbf{G}^{F}, X^{F}\right)$ in Definition 2.11(2), we have Shimura curves embedded diagonally. In fact the diagonal embedding $\mathrm{GL}_{2 . \mathbb{R}} \rightarrow \prod_{\sigma} \mathrm{GL}_{2, \sigma}$ descends to $\mathrm{GL}_{2, \mathbb{Q}} \hookrightarrow \operatorname{Res}_{F / \mathbb{Q}} \mathrm{GL}_{2}$, which has image in $\mathbf{G}^{F}$; the homomorphism $h: \mathbb{S} \rightarrow \mathbf{G}_{\mathbb{R}}^{F}$ used there factors through it, which gives the chain of subdata $\left(\mathrm{GL}_{2}, \mathscr{H}_{1}^{ \pm}\right) \hookrightarrow$ $\left(\mathbf{G}^{F}, X^{F}\right) \hookrightarrow\left(\mathrm{GSp}_{2 g}, \mathscr{H}_{g}^{ \pm}\right)$. Such Shimura curves are called Shimura curves of type I.

Note that the Shimura curves given by different embeddings $\left(\mathrm{GL}_{2}, \mathscr{H}_{1}^{ \pm}\right) \hookrightarrow$ $\left(\mathbf{G}^{F}, X^{F}\right) \hookrightarrow\left(\mathrm{GSp}_{2 g}, \mathscr{H}_{g}^{ \pm}\right)$only differ from each other by Hecke translation using $\mathrm{GSp}_{2 g}(\mathbb{Q})$. For example, if $E$ and $F$ are two totally real étale $\mathbb{Q}$-algebra of dimension $g$ giving rise to $\left(\mathbf{G}^{E}, X^{E}\right)$ and $\left(\mathbf{G}^{F}, X^{F}\right)$ as above, then their embeddings into $\left(\mathrm{GSp}_{2 g}, \mathscr{H}_{g}^{ \pm}\right)$are the same as the choice of $E$-structure (resp. $F$-structure) on the $\mathbb{Q}$-Lagrangian subspace of the underlying symplectic space. The restriction to $\left(\mathrm{GL}_{2}, \mathscr{H}_{1}^{ \pm}\right)$simply treats $E$ (resp. $F$ ) as a $\mathbb{Q}$-vector space of dimension $g$, hence the embedding $\left(\mathrm{GL}_{2}, \mathscr{H}_{1}^{ \pm}\right) \hookrightarrow\left(\mathrm{GSp}_{2 g}, \mathscr{H}_{g}^{ \pm}\right)$always factors through some $\left(\mathbf{G}^{L}, X^{L}\right)$ with $L=\mathbb{Q}^{g}$ (direct product $\mathbb{Q}$-algebra) given by the choice of a basis for a $\mathbb{Q}$-Lagrangian subspace of the symplectic space. Since different $\mathbb{Q}$-Lagrangians are conjugate under $\mathrm{GSp}_{2 g}(\mathbb{Q})$, we see that these embeddings of $\left(\mathrm{GL}_{2}, \mathscr{H}_{1}^{ \pm}\right)$are permuted to each other by Hecke translation.

We mention some facts about Shimura curves of type I:

Lemma 2.13. (1) Let $A$ be a principally polarized abelian variety over $\mathbb{C}$ with $\operatorname{End}^{\circ}(A):=\operatorname{End}(A) \otimes_{\mathbb{Z}} \mathbb{Q}$. Then $\operatorname{End}^{\circ}(A)$ contains the matrix algebra $\operatorname{Mat}_{g}(\mathbb{Q})$ if and only if $A$ is isogenous to a g-fold self-product of some elliptic curve.

(2) Let $A$ be an abelian variety as in (1). Then the point $x_{A}$ on $\mathcal{A}_{g}$ parameterizing A falls in some Hecke translate of the Shimura curve defined by the diagonal embedding $\left(\mathrm{GL}_{2}, \mathscr{H}_{1}^{ \pm}\right) \hookrightarrow\left(\mathrm{G}^{L}, X^{L}\right) \hookrightarrow\left(\mathrm{GSp}_{2 g}, \mathscr{H}_{g}^{ \pm}\right)$, using the trivial real $\mathbb{Q}$-algebra $L=\mathbb{Q}^{g}$. 
Proof. (1) A $g$-dimensional principally polarized abelian variety $A$ (over $\mathbb{C}$ ) admits a decomposition up to isogeny $A \sim \prod_{i=1}^{r} A_{i}^{m_{i}}$ where the $A_{i}$ 's are simple abelian varieties non-isomorphic to each other and $m_{i}>0$ are integers such that $\sum_{i=1}^{r} m_{i}=g$. Hence the algebra of endomorphisms up to isogeny of $A$ is

$$
\operatorname{End}^{\circ}(A) \simeq \prod_{i=1}^{r} \operatorname{Mat}_{m_{i}}\left(D_{i}\right),
$$

where $D_{i}=\operatorname{End}^{\circ}\left(A_{i}\right)$ is a division algebra of finite dimension over $\mathbb{Q}$. The maximal semi-simple split $\mathbb{Q}$-algebra (i.e. a finite product of matrix algebras $\operatorname{Mat}_{d}(\mathbb{Q})$ 's) of $\operatorname{End}^{\circ}(A)$ is equal to $\prod_{i=1}^{r} \operatorname{Mat}_{m_{i}}(\mathbb{Q})$.

If $\operatorname{End}^{\circ}(A)$ contains a split simple $\mathbb{Q}$-algebra of the form $\operatorname{Mat}_{g}(\mathbb{Q})$, then one must have $r=1$ and $m_{1}=g$, which means $A$ is isogenous to a $g$-fold self-product of a single elliptic curve. Conversely, if $A$ is isogenous to $E^{g}$ with $E$ some elliptic curve, then $\operatorname{End}^{\circ}(A)=\operatorname{Mat}_{g}\left(\operatorname{End}^{\circ}(E)\right)$ contains $\operatorname{Mat}_{g}(\mathbb{Q})$.

(2) Let $x \in \mathscr{H}_{g}^{+}$be a point giving the Hodge structure $h: \mathbb{S} \rightarrow \mathrm{GSp}_{2 g, \mathbb{R}} \rightarrow \mathrm{GL}_{V, \mathbb{R}}$ on $V$, which defines a point $\bar{x}=\Gamma x$ on $\Gamma \backslash \mathscr{H}_{g}^{+}$for some congruence subgroup $\Gamma \subset \operatorname{Sp}_{2 g}(\mathbb{Q})$ (say $\Gamma=\Gamma(n)$ principal for some $n$ ). Take $a \in \operatorname{GSp}_{2 g}(\mathbb{Q})_{+}$and consider the Hecke correspondence

$$
\Gamma \backslash \mathscr{H}_{g}^{+} \stackrel{q}{\leftarrow} \Gamma_{a} \backslash \mathscr{H}_{g}^{+} \stackrel{q_{a}}{\rightarrow} \Gamma \backslash \mathscr{H}_{g}^{+}
$$

then by the Definition 2.6 we see that the point $\Gamma a x$ lies in $q_{a}\left(q^{-1}\{\bar{x}\}\right)$.

Applying this to the isogeny $f: E^{g} \rightarrow A$, we get $a=f_{*}: H_{1}\left(E^{g}, \mathbb{Q}\right) \simeq H_{1}(A, \mathbb{Q})$. $H_{1}\left(E^{g}\right)$ and $H_{1}(A, \mathbb{Q})$ correspond to two rational Hodge structure on the $\mathbb{Q}$-vector space $V=\mathbb{Q}^{2 g}$, namely two points $x_{E}$ and $x_{A}$ in $\mathscr{H}_{g}^{+} . a=f_{*}$ is an isomorphism of polarized rational Hodge structure, which gives an element in $\operatorname{GSp}_{2 g}(\mathbb{Q})$, still denoted as $a$. We may choose suitable symplectic bases of $H_{1}\left(E^{g}, \mathbb{Q}\right)$ and $H_{1}(A, \mathbb{Q})$ such that $a \in \mathrm{GSp}_{2 g}(\mathbb{Q})_{+}$. The element $a$ transports $x_{E}$ to $x_{A}$, namely the conjugation of $x_{E}$ by $a$ equals $x_{A}$, hence $\Gamma x_{A}$ is a Hecke translate of $\Gamma x_{E}$ by $a \in \operatorname{GSp}_{2 g}(\mathbb{Q})_{+} \cdot$

The second class of Shimura curves are the Shimura curves of Mumford type constructed from corestrictions of quaternion algebras, the idea of which goes back to 51. We recall briefly the construction given in 69.

Let $F$ be a totally real field of degree $d$ over $\mathbb{Q}$, with $d$ distinct real embeddings $\sigma_{1}, \cdots, \sigma_{d}$, and we use $\sigma=\sigma_{1}$ to identify $F$ as a subfield of $\mathbb{R}$. Let $A$ be a quaternion algebra over $F$, equipped with isomorphisms

$$
\rho_{1}: A \otimes_{\sigma_{1}} \mathbb{R} \simeq \operatorname{Mat}_{2}(\mathbb{R}), \quad \rho_{i}: A \otimes_{\sigma_{i}} \mathbb{R} \simeq \mathbb{H},(i=2, \cdots, d),
$$

with $\mathbb{H}$ Hamilton's quaternion algebra over $\mathbb{R}$. The corestriction $D=\operatorname{Cor}_{F / \mathbb{Q}} A$ is a central simple algebra over $\mathbb{Q}$, which is isomorphic to either

- $\operatorname{Mat}_{2^{d}}(\mathbb{Q})$ and $d$ is odd; or

- $\operatorname{Mat}_{2^{d}}(L)$, for some quadratic extension $L=\mathbb{Q}(\sqrt{b})$ over $\mathbb{Q} ; L$ is imaginary if and only if $d$ is even.

In both cases we have an embedding $D=\operatorname{Cor}_{F / \mathbb{Q}} A \hookrightarrow \operatorname{Mat}_{2^{d+\epsilon}}(\mathbb{Q})$ for $\epsilon \in 0,1$, and we simply write it as $D \subseteq \operatorname{Mat}_{2^{m}}(\mathbb{Q})$ with $m$ minimal.

Write $A^{1}$ for the kernel of the reduced norm Nrd : $A^{\times} \rightarrow F^{\times}$. The $\mathbb{Q}$-group $\mathbf{G}^{\prime}$ associated to $A^{1}$ is connected and semi-simple, with $\mathbf{G}_{\mathbb{R}}^{\prime} \simeq \mathrm{SL}_{2, \mathbb{R}} \times \mathrm{SU}_{2}(\mathbb{R})^{d-1}$. From 69] we know that the homomorphism $A^{\times} \rightarrow D \hookrightarrow \operatorname{Mat}_{2^{m}}(\mathbb{Q})$ defines a representation of $A^{1}$ which preserves a symplectic form on $V=\mathbb{Q}^{2^{m}}$. 
We enlarge $\mathbf{G}^{\prime}$ to a connected reductive $\mathbb{Q}$-group $\mathbf{G}$ which only differs from $\mathbf{G}^{\prime}$ by the split center $\mathbb{G}_{\mathrm{m}, \mathbb{Q}}$. It is the $\mathbb{Q}$-group associated to

$$
A^{*}:=\left\{a \in A: \operatorname{Nrd}(a) \in \mathbb{Q}^{\times} \subset F^{\times}\right\} .
$$

Similar to the case of the affine modular curve $Y(d)$ discussed above, we have the Shimura datum $(\mathbf{G}, X)$, where $X$ is the $\mathbf{G}(\mathbb{R})$-conjugacy class of the following homomorphism $h: \mathbb{S} \rightarrow \mathbf{G}_{\mathbb{R}}$ given by

$$
z=\rho \exp (\sqrt{-1} \theta) \mapsto\left(\left[\begin{array}{cc}
\rho \cos \theta & \rho \sin \theta \\
-\rho \sin \theta & \rho \cos \theta
\end{array}\right], I_{2}, \cdots, I_{2}\right) .
$$

$X$ is isomorphic to $\mathscr{H}_{1}^{ \pm}$, and $(\mathbf{G}, X)$ is a subdatum of $\left(\mathrm{GSp}_{2^{m}}, \mathscr{H}_{2^{m-1}}^{ \pm}\right)$by the representation of $\mathbf{G}$ induced by $A \rightarrow \operatorname{Mat}_{2^{m}}(\mathbb{Q})$.

Write $C_{A}$ for the connected Shimura curve defined by the datum $(\mathbf{G}, X)$ above using suitable level structure and the component $\mathscr{H}_{1}^{+}$, with $\eta$ the generic point of $C_{A}$. The defining symplectic representation of $\mathbf{G}$ gives a universal family of abelian varieties $X_{A} \rightarrow C_{A}$, and its generic fiber $X_{\eta}$ is an abelian variety. The endomorphism algebra of $X_{\eta}$ has been classified in [69], and one of the two following cases holds:

(1) $m=d>1, \operatorname{dim} X_{\eta}=2^{d-1}$ and $\operatorname{End}\left(X_{\eta}\right) \otimes_{\mathbb{Z}} \mathbb{Q}=\mathbb{Q}$;

(2) $m=d+1, \operatorname{dim} X_{\eta}=2^{d}$, and

a. for $d$ odd, $\operatorname{End}\left(X_{\eta}\right) \otimes_{\mathbb{Z}} \mathbb{Q}$ is a totally definite quaternion algebra over $\mathbb{Q}$;

b. for $d$ even, $\operatorname{End}\left(X_{\eta}\right) \otimes_{\mathbb{Z}} \mathbb{Q}$ is a totally definite quaternion algebra over $\mathbb{Q}$.

We remark that for $d=1$ or 2 , there are only two Shimura curves of Mumford type for the given quaternion algebra $A$, and both of them are of PEL type. The curve classifies abelian surfaces resp. abelian fourfolds $X$ with $\operatorname{End}(X) \otimes_{\mathbb{Z}} \mathbb{Q}$ a totally indefinite resp. totally definite quaternion algebra over $\mathbb{Q}$.

2.3. Logarithmic Higgs bundles on curves in $\mathcal{A}_{g}$. Let $\mathcal{A}_{g}=\mathcal{A}_{g,[n]}(n \geq 3)$ be the moduli space of principal polarized abelian varieties with level- $n$ structure and $\overline{\mathcal{A}}_{g} \supseteq \mathcal{A}_{g}$ a smooth toroidal compactification with $\Delta:=\overline{\mathcal{A}}_{g} \backslash \mathcal{A}_{g}$. Note that $\mathcal{A}_{g}$ carries a universal family of abelian varieties (cf. 60] )

$$
\mathfrak{h}: \mathcal{X}_{g} \longrightarrow \mathcal{A}_{g} \text {. }
$$

The relative de Rham bundle $\left(H_{d R}^{1}\left(\mathcal{X}_{g} / \mathcal{A}_{g}\right), \nabla\right)$, together with a polarization and the Hodge filtration $\mathfrak{h}_{*}\left(\Omega_{\mathcal{X}_{0} / \mathcal{A}_{g,[n]}}^{1}\right) \subseteq H_{d R}^{1}\left(\mathcal{X}_{g} / \mathcal{A}_{g}\right)$, forms a polarized variation of Hodge structure (PVHS).

Consider the underlying universal locally constant sheaf $\mathbb{V}=R^{1} \mathfrak{h}_{*} \mathbb{Q}_{\mathcal{X}_{0}}$. If $n$ is large enough, then $\mathbb{V}$ has unipotent local monodromy around all components of the boundary $\Delta$ by [53, $\S 4]$. We will always assume that $\mathbb{V}$ has the property.

The above PVHS has a unique extension over $\overline{\mathcal{A}}_{g,[n]}$, and the extended GaussManin connection $\nabla$ has logarithmic poles along $\Delta$, cf. [59, $\S 11.1, \S 11.2]$. By taking the grading of the extended Hodge filtration, one obtains a logarithmic system of Hodge bundles $\left(E^{1,0} \oplus E^{0,1}, \theta\right)$, where the Higgs field

$$
\theta: E^{1,0} \longrightarrow E^{0,1} \otimes \Omega{\frac{1}{\mathcal{A}_{g}}}(\log \Delta)
$$

is an extension of the following Kodaira-Spencer map on the Hodge bundles

$$
\theta: \mathfrak{h}_{*}\left(\Omega_{\mathcal{X}_{g} / \mathcal{A}_{g}}^{1}\right) \longrightarrow R^{1} \mathfrak{h}_{*} \mathcal{O}_{\mathcal{X}_{g}} \otimes \Omega_{\mathcal{A}_{g}}^{1}
$$

Consider a (smooth) projective curve contained in $\overline{\mathcal{A}}_{g,[n]}$ :

$$
\phi: \bar{C} \hookrightarrow \overline{\mathcal{A}}_{g,[n]}, \quad \text { with } \Delta_{\bar{C}}:=\phi^{-1}(\Delta) \text { a divisor. }
$$


Then by pull-back $C:=\bar{C} \backslash \Delta_{\bar{C}}$ carries a universal family of abelian varieties

$$
h: X \rightarrow C
$$

and a PVHS with underlying local system $\mathbb{V}_{C}:=R^{1} h_{*} \mathbb{Z}_{X}=\phi^{*} \mathbb{V}$, which has unipotent local monodromy around $\Delta_{\bar{C}}$ by assumption. Because of the compatibility of the Deligne's canonical extension with pullback under a morphism, we obtain

$$
\left(E_{\bar{C}}^{1,0} \oplus E_{\bar{C}}^{0,1}, \theta_{\bar{C}}\right)=\phi^{*}\left(E^{1,0} \oplus E^{0,1}, \theta\right) .
$$

In particular, for a Shimura curve $C$, one obtains an associated logarithmic Higgs bundle on the smooth completion $\bar{C} \supseteq C$.

2.4. Families of semi-stable curves. Our main technique will be built on the theory of (one-dimensional) families of semi-stable curves. In the subsection, we would like to review some basic facts and fix the notations, which will be used freely in this paper, cf. 4, 9, 22.

Recall that a semi-stable (resp. stable) curve is a complete connected reduced nodal curve such that each rational component intersects with the other components at $\geq 2$ (resp. 3) points. A semi-stable (resp. stable) family of curves is a flat projective morphism $\bar{f}: \bar{S} \rightarrow \bar{B}$ from a projective surface $\bar{S}$ to a smooth projective curve $\bar{B}$ with connected fibres such that all the singular fibres of $\bar{f}$ are semi-stable (resp. stable) curves. Moreover, $\bar{f}$ is said to be

- a hyperelliptic family if a general fibre of $\bar{f}$ is a hyperelliptic curve;

- isotrivial if all its smooth fibres are isomorphic to each other;

- relatively minimal if no singular fiber of $\bar{f}$ has any $(-1)$-component.

Note that if $\bar{f}$ is semi-stable, then $\bar{f}$ is relatively minimal. From now on, we assume that $\bar{f}: \bar{S} \rightarrow \bar{B}$ is a semi-stable family of curves of genus $g \geq 2$ with singular fibres $\Upsilon \rightarrow \Delta$ and $\bar{S}$ is smooth.

Denote by $\omega_{\bar{S}} / \bar{B}=\omega_{\bar{S}} \otimes \bar{f}^{*} \omega_{\bar{B}}$ the relative canonical sheaf of $\bar{f}$. Let $b=g(\bar{B})$, $p_{g}=h^{0}\left(\bar{S}, \omega_{\bar{S}}\right), q=h^{0}\left(\bar{S}, \Omega \frac{1}{S}\right), \chi\left(\mathcal{O}_{\bar{S}}\right)=p_{g}-q+1$, and $\chi_{\text {top }}(\cdot)$ be the topological Euler characteristic. Consider the following relative invariants:

$$
\left\{\begin{array}{l}
\omega_{\bar{S} / \bar{B}}^{2}=\omega_{\bar{S}}^{2}-8(g-1)(b-1), \\
\delta_{\bar{f}}=\chi_{\text {top }}(\bar{S})-4(g-1)(b-1)=\sum_{F \in \Upsilon} \delta(F), \\
\operatorname{deg} \bar{f}_{*} \omega_{\bar{S} / \bar{B}}=\chi\left(\mathcal{O}_{\bar{S}}\right)-(g-1)(b-1),
\end{array}\right.
$$

where $\delta(F)$ is the number of nodes of $F$. All the invariants in (2-1) are nonnegative and satisfy the Noether's formula:

$$
12 \operatorname{deg} \bar{f}_{*} \omega_{\bar{S} / \bar{B}}=\omega_{\bar{S} / \bar{B}}^{2}+\delta_{\bar{f}}
$$

And $\operatorname{deg} \bar{f}_{*} \omega_{\bar{S} / \bar{B}}=0$ ( or equivalently, $\omega_{\bar{S}}^{2} / \bar{B}=0$ ) if and only if $\bar{f}$ is smooth and isotrivial. Since $\bar{f}$ is semi-stable, we also have

$$
\bar{f}_{*} \omega_{\bar{S} / \bar{B}}=\bar{f}_{*} \Omega \frac{1}{S} / \bar{B}(\log \Upsilon)
$$

where $\bar{f}_{*} \Omega \frac{1}{S} / \bar{B}(\log \Upsilon)$ is defined by the following exact sequence

$$
0 \longrightarrow \bar{f}^{*} \Omega \frac{1}{B}(\log \Delta) \longrightarrow \Omega \frac{1}{S}(\log \Upsilon) \longrightarrow \Omega \frac{1}{S} / \bar{B}(\log \Upsilon) \longrightarrow 0 .
$$


By contracting all $(-2)$-curves contained in singular fibres, one gets a stable family $\bar{f}^{\#}: \bar{S}^{\#} \rightarrow \bar{B}$ and a commutative diagram as below:

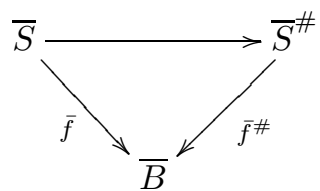

$\bar{S}^{\#}$ is not necessarily smooth. For every singular point $q$ of $\bar{S}^{\#},\left(\bar{S}^{\#}, q\right)$ is a rational double point of type $A_{\lambda_{q}}$ (cf. [4]) with $\lambda_{q}$ the number of (-2)-curves in $\bar{S}$ over $q$.

We are going to define invariants $\left\{\delta_{i}(F) \mid 0 \leq i \leq[g / 2]\right\}$ for a singular fibre $F$ of $\bar{f}$. First, we say a singular point $q$ of $F$ to be of type $i \in[1, g / 2]$ (resp. 0 ) if the partial normalization of $F$ at $q$ consists of two connected components of arithmetic genera $i$ and $g-i$ (resp. is connected). Then we define $\delta_{i}(F)$ to be the number of singular points of type $i$ in $F$. Or alternatively we define $\delta_{i}(F)$ in terms of the stable model $F^{\#} \subseteq \bar{S}^{\#}$. Recall that a singular point $q \in F^{\#}$ is said to have multiplicity $m$ if $\bar{S} \#$ around $q$ is locally of the form $x y=t^{m}$, where $t$ is a local coordinate of $\bar{B}$. Then $\delta_{i}(F)$ is defined to be the number of singular points of type $i$ counting multiplicity in $F^{\#}$. We remark that $\left(\bar{S}^{\#}, q\right)$ is a rational double point of type $A_{m_{q}-1}$, if $m_{q}>1$ is the multiplicity of $q$.

Denote always by $\Upsilon_{c t} \rightarrow \Delta_{c t}$ (resp. $\left.\Upsilon_{n c} \triangleq \Upsilon \backslash \Upsilon_{c t} \rightarrow \Delta_{n c} \triangleq \Delta \backslash \Delta_{c t}\right)$ the singular fibres with compact (resp. non-compact) Jacobian. Define $\delta_{h}(F)=\sum_{i=2}^{[g / 2]} \delta_{i}(F)$, and

$$
\begin{cases}\delta_{i}(\Upsilon)=\sum_{F \in \Upsilon} \delta_{i}(F), & \delta_{i}\left(\Upsilon_{c t}\right)=\sum_{F \in \Upsilon_{c t}} \delta_{i}(F), \quad \delta_{i}\left(\Upsilon_{n c}\right)=\sum_{F \in \Upsilon_{n c}} \delta_{i}(F) . \\ \delta_{h}(\Upsilon)=\sum_{i=2}^{[g / 2]} \delta_{i}(\Upsilon), & \delta_{h}\left(\Upsilon_{c t}\right)=\sum_{i=2}^{[g / 2]} \delta_{i}\left(\Upsilon_{c t}\right) .\end{cases}
$$

Then

$$
\left\{\begin{aligned}
\delta(F) & =\sum_{i=0}^{[g / 2]} \delta_{i}(F)=\delta_{0}(F)+\delta_{1}(F)+\delta_{h}(F) \\
\delta_{\bar{f}} & =\sum_{i=0}^{[g / 2]} \delta_{i}(\Upsilon)=\delta_{0}(\Upsilon)+\delta_{1}(\Upsilon)+\delta_{h}(\Upsilon)
\end{aligned}\right.
$$

When $F \in \Upsilon_{c t}$, each irreducible component of $F$ is smooth. So one can define

$$
l_{i}(F)=\#\{D \subseteq F \mid g(F)=i\}, \quad l_{h}(F)=\sum_{i \geq 2} l_{i}(F) .
$$

Note that the dual graph of $F$ is a tree for $F \in \Upsilon_{c t}$. Hence

$$
\delta_{0}(F)=0, \quad \sum_{j} \delta_{j}(F)=\sum_{i} l_{i}(F)-1, \quad \sum_{i} i \cdot l_{i}(F)=g, \quad \forall F \in \Upsilon_{c t} .
$$

We also want to remark that these invariants $\delta_{i}(\Upsilon)$ 's have the following moduli meanings. Let $\overline{\mathcal{M}}_{g}$ be the moduli space of complex stable curves of genus $g$. By [13, the boundary $\overline{\mathcal{M}}_{g} \backslash \mathcal{M}_{g}$ is of codimension one and has $[g / 2]+1$ irreducible components $\Delta_{0}, \Delta_{1}, \cdots, \Delta_{[g / 2]}$, which define divisor classes in $\operatorname{Pic}\left(\overline{\mathcal{M}}_{g}\right) \otimes \mathbb{Q}$. Note that a general point of $\Delta_{0}$ represents an irreducible stable curve with one node, while a general point of $\Delta_{i}(i>0)$ corresponds to a stable curve consisting of two components of arithmetic genera $i$ and $g-i$ respectively and intersecting at one point. There is also a natural class $\lambda \in \operatorname{Pic}\left(\overline{\mathcal{M}}_{g}\right) \otimes \mathbb{Q}$ called the Hodge class 
with the following property (cf. [13): for every non-isotrivial semi-stable family $\bar{f}: \bar{S} \rightarrow \bar{B}$ with the associated moduli morphism $\varphi: \bar{B} \rightarrow \bar{M}_{g}$, then

$$
\operatorname{deg} \varphi^{*}(\lambda)=\operatorname{deg} \bar{f}_{*} \omega_{\bar{S} / \bar{B}}, \quad \delta_{i}(\Upsilon)=\operatorname{deg} \varphi^{*}\left(\Delta_{i}\right) .
$$

We now assume that $\bar{f}: \bar{S} \rightarrow \bar{B}$ is a semi-stable family of hyperelliptic curves of genus $g \geq 2$ till the end of this subsection. We are going to define invariants

$$
\xi_{j}(\Upsilon)=\sum_{F \in \Upsilon} \xi_{j}(F), \quad \forall 0 \leq j \leq[(g-1) / 2] .
$$

It suffices to define $\xi_{j}(F)$ for singular fibers $F$ of $\bar{f}$.

- First we define the index of a singular point $p$ of a stable $(2 g+2)$-pointed nodal curve $\Gamma$ of arithmetic genus zero. Note that $\Gamma \backslash\{p\}$ consists of two connected components $\Gamma^{\prime}$ and $\Gamma^{\prime \prime}$, which respectively contain $\alpha_{1}$ and $\alpha_{2}$ marked points. Clearly $\alpha_{1}+\alpha_{2}=2 g+2$. We call $\min \left(\alpha_{1}, \alpha_{2}\right)$ to be the index of $p \in \Gamma$.

- Next we describe singular points of a semi-stable hyperelliptic curve $\widetilde{F}$ when $\widetilde{F}$ can be viewed as an admissible double $\psi: \widetilde{F} \rightarrow \Gamma$ over a stable curve $\Gamma$ as above (cf. [9] or [23]). If $p \in \Gamma$ has odd index $2 k+1$, then $\psi$ is branched at $p$ and the unique point $q \in \widetilde{F}$ lying above $p$ is a singular point of type $k$. If $p \in \Gamma$ has even index $2 k+2$, then $\psi$ is unbranched at $p$ and two points $q^{\prime}, q^{\prime \prime} \in \widetilde{F}$ lying above $p$ are of type 0 . Define invariants

$$
\begin{aligned}
& \xi_{0}(\widetilde{F}):=2 \cdot \#\{\text { singular points in } \Gamma \text { of index } 2\}, \\
& \xi_{j}(\widetilde{F}):=\#\{\text { singular points in } \Gamma \text { of index } 2 j+2\}, \quad 1 \leq j \leq[(g-1) / 2] .
\end{aligned}
$$

- Finally we define $\xi_{j}(F)$ for any singular fiber $F$ of $\bar{f}$. Let $\tilde{f}: \widetilde{S} \rightarrow \widetilde{B}$ be the semi-stable family corresponding to the base change of $\bar{f}$ with respect to a finite morphism $\pi: \widetilde{B} \rightarrow \bar{B}$ of degree $d$. When $d>>0$, the pre-image $\widetilde{F}$ of $F$ is an admissible double cover of a stable $(2 g+2)$-pointed nodal curve $\widetilde{\Gamma}$ of arithmetic genus zero. Then define

$$
\xi_{j}(F)=\frac{\xi_{j}(\widetilde{F})}{d}, \quad \forall 0 \leq j \leq[(g-1) / 2] .
$$

Clearly the definition of $\xi_{j}(F)$ is independent of the choice of $\pi$. In particular we have

$$
\delta_{0}(F)=\xi_{0}(F)+2 \sum_{j=1}^{[(g-1) / 2]} \xi_{j}(F) .
$$

Let $\mathcal{H}_{g} \subseteq \mathcal{M}_{g}$ (resp. $\overline{\mathcal{H}}_{g} \subseteq \overline{\mathcal{M}}_{g}$ ) be the moduli space of smooth (resp. stable) hyperelliptic complex curves of genus $g$. By $\left[9, \Delta_{i} \cap \overline{\mathcal{H}}_{g}\right.$ is an irreducible divisor of $\overline{\mathcal{M}}_{g}$, also denoted by $\Delta_{i} ; \Delta_{0} \cap \overline{\mathcal{H}}_{g}$ is not irreducible, actually

$$
\Delta_{0} \cap \overline{\mathcal{H}}_{g}=\Xi_{0} \cup \Xi_{1} \cup \cdots \cup \Xi_{[(g-1) / 2]},
$$

where $\Xi_{0}$ consists of irreducible stable hyperelliptic curves with a unique node, and for $1 \leq j \leq[(g-1) / 2]$, a general point of $\Xi_{j}$ represents a stable curve consisting of two hyperelliptic curves intersecting at two points and respectively of genera $j$ and $g-j-1$. As divisors (cf. [9]),

$$
h^{*}\left(\Delta_{0}\right)=\Xi_{0}+2 \sum_{j=1}^{[(g-1) / 2]} \Xi_{j}, \quad \text { where } h: \overline{\mathcal{H}}_{g} \hookrightarrow \overline{\mathcal{M}}_{g} \text { is the embedding. }
$$


Assume that $\bar{f}: \bar{S} \rightarrow \bar{B}$ is a non-isotrivial semi-stable family of hyperelliptic curves and $\varphi: \bar{B} \rightarrow \overline{\mathcal{H}}_{g}$ is the induced map, then

$$
\begin{cases}\xi_{j}(\Upsilon)=\operatorname{deg} \varphi^{*}\left(\Xi_{j}\right), & \forall 0 \leq j \leq[(g-1) / 2] ; \\ \delta_{0}(\Upsilon)=\operatorname{deg} \varphi^{*}\left(\Xi_{0}\right)+2 \sum_{j=1}^{[(g-1) / 2]} \operatorname{deg} \varphi^{*}\left(\Xi_{j}\right) ; & \\ \delta_{i}(\Upsilon)=\operatorname{deg} \varphi^{*}\left(\Delta_{i}\right), & \forall 1 \leq i \leq[g / 2] .\end{cases}
$$

\section{FAmily of SEMi-StABle CURVES REPRESENTing A CURVE IN $\mathcal{T}_{g}$}

Given a smooth closed curve $C \Subset \mathcal{T}_{g}$ with a suitable smooth compactification $\bar{C}$ by joining a finite set of cusps $\Delta_{\bar{C}}$, we would like to construct a family $\bar{f}: \bar{S} \rightarrow \bar{B}$ of semi-stable curves representing $C$ in the section. We also investigate the exact relation between the associated logarithmic Higgs bundles over $\bar{B}$ and $\bar{C}$.

Fix an integer $n$, let $\mathcal{M}_{g}^{c t}=\mathcal{M}_{g,[n]}^{c t} \supseteq \mathcal{M}_{g}=\mathcal{M}_{g,[n]}$ be the partial compactification of the moduli space of smooth projective genus- $g$ curves with level- $n$ structure by adding stable curves with compact Jacobians. When $n \geq 3$, it carries a universal family of stable curves with compact Jacobians (cf. 60] )

$$
\mathfrak{f}: \mathcal{S}_{g}^{c t} \longrightarrow \mathcal{M}_{g}^{c t}
$$

The Torelli morphism $j^{\mathrm{o}}$ can be naturally extended to $\mathcal{M}_{g}^{c t}$ :

$$
j: \mathcal{M}_{g}^{c t} \longrightarrow \mathcal{A}_{g}, \quad \text { with } \mathcal{T}_{g}=j\left(\mathcal{M}_{g}^{c t}\right) .
$$

The morphism $j^{\circ}$ is $2: 1$ and ramified exactly on the locus of hyperelliptic curves (cf. [56]). However the relative dimension of $j$ is positive along the boundary $\mathcal{T}_{g} \backslash \mathcal{T}_{g}^{\mathrm{o}}$.

Let $B$ be the normalization of the strict inverse image $j^{-1}(C)$ of $C$, and denote by $j_{B}: B \rightarrow C$ the induced morphism. If $B$ is reducible, then replace $B$ by one irreducible component. By pulling back the universal family $\mathfrak{f}: \mathcal{S}_{g}^{c t} \rightarrow \mathcal{M}_{g}^{c t}$ to $B$ and resolving singularities, one gets a family $f: S \rightarrow B$ of semi-stable curves that extends uniquely to a family $\bar{f}: \bar{S} \rightarrow \bar{B}$ of semi-stable curves over the smooth completion $\bar{B} \supseteq B$.

Definition 3.1. The family $\bar{f}: \bar{S} \rightarrow \bar{B}$ is called the family of semi-stable curves representing $C \subseteq \mathcal{T}_{g}$ via the Torelli morphism.

Let $h: X \rightarrow C$ be the universal family in Section 2.3. By the construction of the Torelli morphism, we obtain the following

Proposition 3.2. Let $j a c(f): \operatorname{Jac}(S / B) \longrightarrow B$ denote the relative Jacobian of the family $f: S \rightarrow B$ and $j_{B}: B \rightarrow C$ the induced morphism as above. Then

$$
(j a c(f): \operatorname{Jac}(S / B) \longrightarrow B)=j_{B}^{*}(h: X \longrightarrow C) .
$$

In particular, $\mathbb{V}_{B}:=R^{1} j a c(f)_{*} \mathbb{Q}_{J a c(S / B)}=j_{B}^{*} \mathbb{V}_{C}$.

It is well-known that the logarithmic Higgs bundle associated to $\mathbb{V}_{B}$ has the form

$$
\left(E_{\bar{B}}^{1,0} \oplus E_{\bar{B}}^{0,1}, \theta_{\bar{B}}\right)=\left(\bar{f}_{*} \omega_{\bar{S} / \bar{B}} \oplus R^{1} \bar{f}_{*} \mathcal{O}_{\bar{S}}, \theta_{\bar{B}}\right),
$$

and it admits a decomposition of Higgs bundles similarly to (1-1):

$$
\left(E_{\bar{B}}^{1,0} \oplus E_{\bar{B}}^{0,1}, \theta_{\bar{B}}\right)=\left(A_{\bar{B}}^{1,0} \oplus A_{\bar{B}}^{0,1},\left.\theta_{\bar{B}}\right|_{A_{\bar{B}}^{1,0}}\right) \oplus\left(F_{\bar{B}}^{1,0} \oplus F_{\bar{B}}^{0,1}, 0\right) .
$$


Since both $\bar{B}$ and $\bar{C}$ are smooth projective curves, the morphism $j_{B}: B \rightarrow C$ extends to a morphism $\bar{j}_{B}: \bar{B} \rightarrow \bar{C}$ such that $\Delta_{n c}:=\bar{B} \backslash B=\bar{j}_{B}^{-1}\left(\Delta_{\bar{C}}\right)$ and $j_{B}^{*}\left(\mathbb{V}_{C}\right)=\mathbb{V}_{B}$. Hence

$$
\bar{j}_{B}^{*}\left(E_{\bar{C}}^{1,0} \oplus E_{\bar{C}}^{0,1}, \theta_{\bar{C}}\right)=\left(E_{\bar{B}}^{1,0} \oplus E_{\bar{B}}^{0,1}, \theta_{\bar{B}}\right) .
$$

In particular,

$$
\bar{j}_{B}^{*}\left(E_{\bar{C}}^{1,0}\right)=E_{\bar{B}}^{1,0}=\bar{f}_{*} \omega_{\bar{S} / \bar{B}}, \quad \bar{j}_{B}^{*}(A \bar{C})=A \frac{1,0}{B} .
$$

Definition 3.3. Let $\bar{f}: \bar{S} \rightarrow \bar{B}$ be any family of semi-stable curves of genus $g \geq 2$ and $\Upsilon_{n c} \rightarrow \Delta_{n c}$ the singular fibres with non-compact Jacobian. Then $\bar{f}$ is said to be with maximal Higgs field if

$$
\left.\theta_{\bar{B}}\right|_{A_{\bar{B}}^{1,0}}: A_{\bar{B}}^{1,0} \longrightarrow A_{\bar{B}}^{0,1} \otimes \Omega \frac{1}{B}\left(\log \Delta_{n c}\right)
$$

is an isomorphism, and to be with strictly maximal Higgs field if furthermore $F_{\bar{B}}^{1,0} \oplus$ $F_{\bar{B}}^{0,1}=0$. By $[68], \bar{f}$ has strictly maximal (resp. maximal) Higgs field if and only if

$$
\begin{aligned}
\operatorname{deg} \bar{f}_{*} \omega_{\bar{S} / \bar{B}} & =\frac{g}{2} \cdot \operatorname{deg} \Omega \frac{1}{B}\left(\log \Delta_{n c}\right), \\
\left(\operatorname{resp} . \quad \operatorname{deg} \bar{f}_{*} \omega_{\bar{S} / \bar{B}}\right. & \left.=\frac{\operatorname{rank} A \frac{1,0}{B}}{2} \cdot \operatorname{deg} \Omega \frac{1}{B}\left(\log \Delta_{n c}\right)\right) .
\end{aligned}
$$

For a smooth closed curve $C \Subset \mathcal{T}_{g}$, we have given two definitions respectively regarding the (strict) maximality of the Higgs fields $\theta_{\bar{C}}$ and $\theta_{\bar{B}}$. To understand the relation between them, we start with the following:

Proposition 3.4. Let $\bar{f}: \bar{S} \rightarrow \bar{B}$ be the family of semi-stable curves representing a smooth closed curve $C \Subset \mathcal{T}_{g} \subseteq \mathcal{A}_{g}$ as above. Then $\operatorname{rank} A_{\bar{B}}^{1,0}=\operatorname{rank} A_{\bar{C}}^{1,0}$, and

(i). if $C \Subset \mathcal{T H} \mathcal{H}_{g}$, then

$$
\operatorname{deg} \bar{f}_{*} \omega_{\bar{S} / \bar{B}}=\operatorname{deg} E_{\bar{C}}^{1,0}, \quad \operatorname{deg} \Omega \frac{1}{B}\left(\log \Delta_{n c}\right)=\operatorname{deg} \Omega_{\bar{C}}\left(\log \Delta_{\bar{C}}\right) ;
$$

(ii). if $C \nsubseteq \mathcal{T H}_{g}$, then

$$
\operatorname{deg} \bar{f}_{*} \omega_{\bar{S}} / \bar{B}=2 \operatorname{deg} E_{\bar{C}}^{1,0}, \quad \operatorname{deg} \Omega \frac{1}{B}\left(\log \Delta_{n c}\right)=2 \operatorname{deg} \Omega_{\bar{C}}\left(\log \Delta_{\bar{C}}\right)+|\Lambda|,
$$

where $\Lambda$ is the ramification locus of the induced cover $j_{B}: B \rightarrow C$.

Proof. By (3-4), it is clear that $\operatorname{rank} A_{\bar{B}}^{1,0}=\operatorname{rank} A_{\bar{C}}^{1,0}$. Note that the Torelli morphism $j^{\circ}: \mathcal{M}_{g} \rightarrow \mathcal{A}_{g}$ is a 2-to-1 morphism ramified exactly on the hyperelliptic locus $\mathcal{H}_{g}$. Hence if $C \Subset \mathcal{T} \mathcal{H}_{g}$, then $j_{B}: B \rightarrow C$ is an isomorphism, and so is $\bar{j}_{B}: \bar{B} \rightarrow \bar{C}$. Thus (3-6) follows from (3-4).

Suppose $C \nsubseteq \mathcal{T} \mathcal{H}_{g}$; then $j^{-1}(C) \rightarrow C$ is a 2-to-1 morphism. If $j^{-1}(C)$ is reducible, then $B$ is the normalization of one of irreducible components of $j^{-1}(C)$. So $B \cong C, \bar{B} \cong \bar{C}$, and (3-7) follows from (3-4). If $j^{-1}(C)$ is irreducible, then $\bar{j}_{B}: \bar{B} \rightarrow \bar{C}$ is a double cover. So by (3-4), $\operatorname{deg} \bar{f}_{*} \omega_{\bar{S} / \bar{B}}=2 \operatorname{deg} E_{\bar{C}}^{1,0}$; and since $\Delta_{n c}=\bar{j}_{B}^{-1}\left(\Delta_{\bar{C}}\right)$, by Hurwitz formula for sheaves of logarithmic 1-forms, one has

$$
\operatorname{deg} \Omega \frac{1}{B}\left(\log \Delta_{n c}\right)=2 \operatorname{deg} \Omega \frac{1}{C}\left(\log \Delta_{\bar{C}}\right)+|\Lambda| .
$$

This completes the proof.

Corollary 3.5 (Hyperelliptic locus). If $C \Subset \mathcal{T} \mathcal{H}_{g}$, then $\theta_{\bar{C}}$ is strictly maximal (resp. maximal) if and only if $\theta_{\bar{B}}$ is strictly maximal (resp. maximal). 
Corollary 3.6 (Non-hyperelliptic locus). Suppose $C \Subset \mathcal{T}_{g,[n]}$, but $C \nsubseteq \mathcal{T H}_{g}$. Then

(i). If $\theta_{\bar{B}}$ is strictly maximal (resp. maximal), then $\theta_{\bar{C}}$ is strictly maximal (resp. maximal);

(ii). Conversely, if $\theta_{\bar{C}}$ strictly maximal (resp. maximal), then

$$
\begin{aligned}
& \operatorname{deg} \bar{f}_{*} \omega_{\bar{S} / \bar{B}}=\frac{g}{2} \cdot \operatorname{deg} \Omega \frac{1}{B}\left(\log \Delta_{n c}\right)-\frac{g}{2} \cdot|\Lambda|, \\
&\left(\text { resp. } \quad \operatorname{deg} \bar{f}_{*} \omega_{\bar{S} / \bar{B}}=\frac{\operatorname{rank} A_{\bar{B}}^{1,0}}{2} \cdot \operatorname{deg} \Omega \frac{1}{B}\left(\log \Delta_{n c}\right)-\frac{\operatorname{rank} A_{\bar{B}}^{1,0}}{2} \cdot|\Lambda|\right),
\end{aligned}
$$

\section{The strict Arakelov inequalities and proofs of the main Results}

In the section, we study the strict Arakelov inequalities, i.e. Theorem 1.4. The main Theorems A, D and E are immediate consequences of these inequalities and the numerical characterization of Shimura curves and totally geodesic curves (cf. Theorem 1.3).

We first recall the Miyaoka-Yau type inequality and sharp slope inequality for a family of semi-stable curves in Section 4.1 from which we deduce the strict Arakelov inequalities in Section 4.2. The proofs of these two types of inequalities are postponed to Sections 5 and 6 respectively.

4.1. Two types of inequalities for a family of semi-stable curves. We state the two types of inequalities as the following theorems.

Theorem 4.1 (Miyaoka-Yau type inequality I, cf. Section[5.1). Let $\bar{f}: \bar{S} \rightarrow \bar{B}$ be a non-isotrivial family of semi-stable curves of genus $g \geq 2$. Then

$$
\omega \frac{2}{S} / \bar{B} \leq(2 g-2) \cdot \operatorname{deg}\left(\Omega \frac{1}{B}\left(\log \Delta_{n c}\right)\right)+2 \delta_{1}\left(\Upsilon_{c t}\right)+3 \delta_{h}\left(\Upsilon_{c t}\right) .
$$

Moreover, if $\Delta_{n c} \neq \emptyset$ or $\Delta=\emptyset$, then the above inequality is strict.

The proof of Theorem 4.1 is based on a theorem of Miyaoka (cf. [41) for the bound on the number of quotient singularities in a surface plus base change technique. Recently Peters (cf. [57]) has informed us that he has a simplified proof by using Cheng-Yau's theorem for a log surface instead of Miyaoka's.

Theorem 4.2 (Moriwaki's Sharp slope inequality, cf. 49 and Section6.1). Let $\bar{f}: \bar{S} \rightarrow \bar{B}$ be the same as in Theorem 4.1. Then

$$
\omega_{\bar{S} / \bar{B}}^{2} \geq \frac{4(g-1)}{g} \cdot \operatorname{deg} \bar{f}_{*} \omega_{\bar{S} / \bar{B}}+\frac{3 g-4}{g} \delta_{1}(\Upsilon)+\frac{7 g-16}{g} \delta_{h}(\Upsilon) .
$$

Theorem 4.3 (Sharp slope inequality I, cf. Section 6.2). Let $\bar{f}: \bar{S} \rightarrow \bar{B}$ be the same as in Theorem 4.1 and $q_{\bar{f}}=q(\bar{S})-g(\bar{B})$ the relative irregularity. If $\bar{f}$ is hyperelliptic, then

$$
\begin{aligned}
\omega_{\bar{S} / \bar{B}}^{2} \geq & \frac{4(g-1)}{g-q_{\bar{f}}} \cdot \operatorname{deg} \bar{f}_{*} \omega_{\bar{S} / \bar{B}}+ \\
& \begin{cases}\frac{3 g^{2}-\left(8 q_{\bar{f}}+1\right) g+10 q_{\bar{f}}-4}{(g+1)\left(g-q_{\bar{f}}\right)} \delta_{1}(\Upsilon) & \text { if } \Delta_{n c} \neq \emptyset ; \\
+\frac{7 g^{2}-\left(16 q_{\bar{f}}+9\right) g+34 q_{\bar{f}}-16}{(g+1)\left(g-q_{\bar{f}}\right)} \delta_{h}(\Upsilon), & \\
\sum_{i=1}^{[g / 2]}\left(\frac{4\left(2 g+1-3 q_{\bar{f}}\right) i(g-i)}{(2 g+1)\left(g-q_{\bar{f}}\right)}-1\right) \delta_{i}(\Upsilon), & \text { if } \Delta_{n c}=\emptyset .\end{cases}
\end{aligned}
$$


Moreover, if $\Delta_{n c}=\emptyset$ and $q_{\bar{f}} \geq 2$, then

$$
\sum_{i=q_{\bar{f}}}^{[g / 2]} \frac{(2 i+1)(2 g+1-2 i)}{g+1} \cdot \delta_{i}(\Upsilon) \geq \sum_{i=1}^{q_{\bar{f}}-1} 4 i(2 i+1) \cdot \delta_{i}(\Upsilon) .
$$

While Theorem 4.2 is a direct consequence of Moriwaki's theorem (cf. 499), Theorem 4.3 is proved based on formulas given by Cornalba and Harris (cf. [9]). The observation that the smooth double cover induced by the hyperelliptic involution is fibred when $q_{\bar{f}}>0$ plays a crucial role.

To get the strict Arakelov inequality for a smooth closed curve $C \Subset \mathcal{T}_{g,[n]}$, we need to deal with the family $\bar{f}: \bar{S} \rightarrow \bar{B}$ of semi-stable curves representing $C$; in this case, the existence of the ramification locus $\Lambda$ of the Torelli morphism $j_{B}: B \rightarrow C$ is the main difficulty and we need a modified version of the above two types of inequalities.

Theorem 4.4 (Miyaoka-Yau type inequality II, cf. Section 5.1). Let $\bar{f}: \bar{S} \rightarrow \bar{B}$ be the family of semi-stable genus-g curves representing a smooth closed curve $C \Subset \mathcal{T}_{g}$ such that $C \nsubseteq \mathcal{T H}_{g}$. For any $p \in \bar{B}$, let $F_{p}=f^{-1}(p)$. If $g \geq 7$, then

$$
\begin{aligned}
\omega \frac{2}{\bar{S} / \bar{B}} \leq & (2 g-2) \cdot \operatorname{deg} \Omega \frac{1}{\bar{B}}\left(\log \Delta_{n c}\right)+ \\
& \sum_{p \in \Delta_{c t} \cap \Lambda} \frac{3}{2}\left(l_{h}\left(F_{p}\right)+l_{1}\left(F_{p}\right)-1\right)+\sum_{p \in \Delta_{c t} \backslash \Lambda}\left(3 l_{h}\left(F_{p}\right)+2 l_{1}\left(F_{p}\right)-3\right) .
\end{aligned}
$$

Moreover, if $\Delta_{n c} \neq \emptyset$ or $\Delta=\emptyset$, then the above inequality is strict.

Theorem 4.5 (Sharp slope inequality II, cf. Section6.3). Let $\bar{f}: \bar{S} \rightarrow \bar{B}$ be the same as in Theorem 4.4. If $g \geq 3$ and $\bar{f}_{*} \omega_{\bar{S} / \bar{B}}$ is a semi-stable vector bundle, then

$$
\begin{aligned}
\omega \frac{2}{S} / \bar{B} \geq & \frac{5 g-6}{g} \operatorname{deg} \bar{f}_{*} \omega_{\bar{S} / \bar{B}}+2(g-2) \cdot|\Lambda|+ \\
& \sum_{p \in \Delta_{c t} \cap \Lambda} 2\left(l_{h}\left(F_{p}\right)+l_{1}\left(F_{p}\right)-1\right)+\sum_{p \in \Delta_{c t} \backslash \Lambda}\left(3 l_{h}\left(F_{p}\right)+2 l_{1}\left(F_{p}\right)-3\right) .
\end{aligned}
$$

The proof of Theorem 4.4 is the same as that of Theorem 4.1, while Theorem 4.5 is proved relying on the derivative of the Torelli morphism, i.e. the second multiplication map $\varrho: S^{2}\left(\bar{f}_{*} \omega_{\bar{S} / \bar{B}}\right) \rightarrow \bar{f}_{*}\left(\omega \frac{\otimes 2}{\bar{S} / \bar{B}}\right)$.

4.2. The strict Arakelov inequalities. In this subsection, we prove Theorem 1.4.

As explained at the end of Section 1.4 the main technique is the theory of fibred surfaces. Given a smooth closed curve $C \Subset \mathcal{T}_{g} \subseteq \mathcal{A}_{g}$, we have constructed a family $\bar{f}: \bar{S} \rightarrow \bar{B}$ of semi-stable curves representing $C$ in Section 3 . We have also established in Proposition 3.4 the relation between the associated Higgs bundles over $\bar{B}$ and $\bar{C}$, where $\bar{C}$ is a suitable smooth compactification of $C$ by joining a finite set of cusps $\Delta_{\bar{C}}$. Hence one can first prove a strict Arakelov inequality for the family $\bar{f}$, and then derive the inequality on $C$ by using Proposition 3.4 ,

However, it turns out that the proof of (1-3) will be more complicated when the ramification locus $\Lambda$ of the double cover $j_{B}: B \rightarrow C$ is not empty. To illustrate the idea, we consider first the easier case $\Lambda=\emptyset$, in which case the invariants involved on $\bar{B}$ and $\bar{C}$ are all proportional by Proposition 3.4. Hence (1-3) follows easily from the following strict Arakelov inequality for a family of semi-stable curves.

Theorem 4.6. Let $\bar{f}: \bar{S} \rightarrow \bar{B}$ be a non-isotrivial family of semi-stable curves of genus $g>4$. Then

$$
\operatorname{deg} \bar{f}_{*} \omega_{\bar{S} / \bar{B}}<\frac{g}{2} \cdot \operatorname{deg} \Omega \frac{1}{B}\left(\log \Delta_{n c}\right) .
$$


Proof. Note that $0 \leq \delta_{1}\left(\Upsilon_{c t}\right) \leq \delta_{1}(\Upsilon)$ and $0 \leq \delta_{h}\left(\Upsilon_{c t}\right) \leq \delta_{h}(\Upsilon)$. Hence by (4-1) and (4-2), one gets

$$
\operatorname{deg} \bar{f}_{*} \omega_{\bar{S} / \bar{B}} \leq \frac{g}{2} \cdot \operatorname{deg} \Omega \frac{1}{B}\left(\log \Delta_{n c}\right)-\frac{g-4}{g} \cdot\left(\delta_{1}(\Upsilon)+4 \delta_{h}(\Upsilon)\right)
$$

Thus (4-7) follows if either $\delta_{1}(\Upsilon)>0$ or $\delta_{h}(\Upsilon)>0$. Suppose $\delta_{1}(\Upsilon)=\delta_{h}(\Upsilon)=0$. Then either $\Delta_{n c} \neq \emptyset$ or $\Delta=\emptyset$. Therefore (4-8) is strict since (4-1) is so, which implies that (4-7) also holds in this case.

Now we are going to prove the strict Arakelov inequalities in general case. The proof of (1-3) requires a strong version of Arakelov inequality for the family $\bar{f}$ by taking the ramification locus $\Lambda$ into account; and the proof of (1-4) relies highly on the strong slope inequality (4-3) with positive relative irregularity.

Proof of Theorem 1.4. (i). We prove (1-3) by contradiction. Suppose (1-3) does not hold. According to [16], one has the following Arakelov inequality:

$$
\operatorname{deg} E_{\bar{C}}^{1,0} \leq \frac{g}{2} \cdot \operatorname{deg} \Omega_{\bar{C}}^{1}\left(\log \Delta_{\bar{C}}\right) .
$$

Hence we may assume that the equality holds in (4-9). By Corollary 3.6, in order to derive a contradiction, it is necessary and sufficient to prove the following stronger version of Arakelov inequality for the family $\bar{f}$.

$$
\operatorname{deg} \bar{f}_{*} \omega_{\bar{S} / \bar{B}}<\frac{g}{2} \cdot\left(\operatorname{deg} \Omega \frac{1}{B}\left(\log \Delta_{n c}\right)-|\Lambda|\right) .
$$

The equality of (4-9) means that the associated Higgs bundle $\left(E_{\bar{C}}^{1,0} \oplus E_{\bar{C}}^{0,1}, \theta_{\bar{C}}\right)$ has strictly maximal Higgs field (cf. (1-2) ). Hence by [69, Proposition 1.2], $E_{\bar{C}}^{1,0}$ is poly-stable; in particular, it is semi-stable. According to (3-4) and [35, Lemma 6.4.12], $\bar{f}_{*} \omega_{\bar{S} / \bar{B}}$ is also semi-stable. Hence one can apply Theorems 4.4 and 4.5 to the family $\bar{f}$, and obtains

$$
\operatorname{deg} \bar{f}_{*} \omega_{\bar{S} / \bar{B}} \leq \frac{2(g-1) g}{5 g-6} \cdot\left(\operatorname{deg} \Omega \frac{1}{B}\left(\log \Delta_{n c}\right)-|\Lambda|\right)+\frac{2 g}{5 g-6} \cdot|\Lambda| .
$$

Since $\delta_{\bar{f}} \geq 0$, according to (2-2) and (4-6), one gets

$$
|\Lambda| \leq \frac{7 g+6}{2(g-2) g} \cdot \operatorname{deg} \bar{f}_{*} \omega_{\bar{S} / \bar{B}}
$$

Combing (4-11) with (4-12), one obtains

$$
\begin{aligned}
\operatorname{deg} \bar{f}_{*} \omega_{\bar{S} / \bar{B}} & \leq \frac{2(g-1)(g-2) g}{5 g^{2}-23 g+6} \cdot\left(\operatorname{deg} \Omega \frac{1}{B}\left(\log \Delta_{n c}\right)-|\Lambda|\right) \\
& =\left(\frac{g}{2}-\frac{\left(g^{2}-11 g+2\right) g}{2\left(5 g^{2}-23 g+6\right)}\right) \cdot\left(\operatorname{deg} \Omega \frac{1}{B}\left(\log \Delta_{n c}\right)-|\Lambda|\right)
\end{aligned}
$$

Since $g>11$, (4-10) follows. The proof is complete.

(ii). To start the proof, one needs a statement below to compare $\operatorname{rank} F_{\bar{B}}^{1,0}$ with $q_{\bar{f}}$, whose proof is postponed to Section 7

Theorem 4.7. Let $\bar{f}: \bar{S} \rightarrow \bar{B}$ be a non-isotrivial family of semi-stable hyperelliptic curves of genus $g \geq 2$. Then after passing to a finite étale base change, one has

$$
\operatorname{rank} F_{\bar{B}}^{1,0}=q_{\bar{f}} .
$$


By the above theorem, we may assume $q_{\bar{f}}=\operatorname{rank} F_{\bar{B}}^{1,0}=g-\operatorname{rank} A_{\bar{B}}^{1,0}$. Combining (4-1) with (4-3), we obtain that if $\Delta_{n c} \neq \emptyset$, then

$$
\operatorname{deg} \bar{f}_{*} \omega_{\bar{S} / \bar{B}}<\frac{\operatorname{rank} A \frac{1,0}{B}}{2} \cdot \operatorname{deg} \Omega \frac{1}{B}\left(\log \Delta_{n c}\right)-\left(\alpha_{1} \delta_{1}(\Upsilon)+\alpha_{h} \delta_{h}(\Upsilon)\right)
$$

and if $\Delta_{n c}=\emptyset$, then

$$
\operatorname{deg} \bar{f}_{*} \omega_{\bar{S} / \bar{B}} \leq \frac{\operatorname{rank} A_{\bar{B}}^{1,0}}{2} \cdot \operatorname{deg} \Omega \frac{1}{B}\left(\log \Delta_{n c}\right)-\sum_{i=1}^{[g / 2]} \beta_{i} \delta_{i}(\Upsilon)
$$

where

$$
\begin{aligned}
\alpha_{1} & =\frac{g^{2}-\left(6 q_{\bar{f}}+3\right) g+12 q_{\bar{f}}-4}{4(g+1)(g-1)}, \\
\alpha_{h} & =\frac{4 g^{2}-\left(13 q_{\bar{f}}+12\right) g+37 q_{\bar{f}}-16}{4(g+1)(g-1)}, \\
\beta_{1} & =\frac{2 g+1-3 q_{\bar{f}}}{2 g+1}-\frac{3\left(g-q_{\bar{f}}\right)}{4(g-1)}, \\
\beta_{i} & =\frac{\left(2 g+1-3 q_{\bar{f}}\right) i(g-i)}{(2 g+1)(g-1)}-\frac{g-q_{\bar{f}}}{g-1}, \quad \forall 2 \leq i \leq[g / 2] .
\end{aligned}
$$

Since $\bar{f}$ is hyperelliptic, one gets (cf. [9, Proposition 4.7])

$$
\delta_{i}(\Upsilon) \text { 's are non-negative, and one of them is positive if } \Delta_{n c}=\emptyset .
$$

Hence it is reasonable to imagine that the strict Arakelov inequality (1-4) holds when $g$ is large enough. The detailed proof is divided into the two following cases:

Case I. $\Delta_{n c} \neq \emptyset$. We prove by contradiction in the case.

Assume that the strict Arakelov inequality (1-4) does not hold. Then by (3-6) and the classical Arakelov inequality (cf. [16]), we must have

$$
\operatorname{deg} \bar{f}_{*} \omega_{\bar{S} / \bar{B}}=\operatorname{deg} E_{\bar{C}}^{1,0}=\frac{\operatorname{rank} A_{\bar{B}}^{1,0}}{2} \cdot \operatorname{deg} \Omega \frac{1}{B}\left(\log \Delta_{n c}\right)=\frac{g-q_{\bar{f}}}{2} \cdot \operatorname{deg} \Omega \frac{1}{B}\left(\log \Delta_{n c}\right) .
$$

Combing this with [37, Corollary 1.7] and the discussion after it, one gets that $g(F)=q_{\bar{f}}$ for every fibre $F$ over $\Delta_{n c}(\neq \emptyset)$, where $g(F)$ is the geometrical genus of $F$. Hence by Proposition 6.5. proved later, we obtain that $q_{\bar{f}} \leq 1$, from which it follows that the coefficients $\alpha_{1}$ and $\alpha_{h}$ in (4-14) are positive since $g>7$. By (4-16), this is a contradiction.

Case II. $\Delta_{n c}=\emptyset$. First, we claim that $q_{\bar{f}} \leq \frac{g-1}{2}$ in this case. Indeed, by $[72$, Theorem 1] or Proposition 6.5, for any hyperelliptic family, one has $q_{\bar{f}} \leq \frac{g+1}{2}$, and if the equality holds then $\bar{f}$ is isotrivial. Hence $q_{\bar{f}} \leq g / 2$. However, if $q_{\bar{f}}=g / 2$, then $\omega_{\bar{S}}^{2} / \bar{B}=\frac{8(g-1)}{g} \cdot \operatorname{deg} \bar{f}_{*} \omega_{\bar{S} / \bar{B}}$ by [72, Theorem 2(a)]. Combining this with (4-3), one obtains that $\delta_{i}(\Upsilon)=0$ for all $i \geq 1$, which contradicts (4-16).

If $q_{\bar{f}}<\frac{(g-4)(2 g+1)}{3(2 g-5)}$, then it is easy to show that $\beta_{i}>\beta_{1}>0$ for any $2 \leq i \leq[g / 2]$. Hence (1-4) follows from (4-15) together with (4-16).

Thus we may assume $q_{\bar{f}} \geq \frac{(g-4)(2 g+1)}{3(2 g-5)}$, from which it follows that $\beta_{1} \leq 0$ and $q_{\bar{f}} \geq 2$ since $g>7$. So according to (4-4) and (4-15), we obtain

$$
\operatorname{deg} \bar{f}_{*} \omega_{\bar{S} / \bar{B}} \leq \frac{\operatorname{rank} A_{\bar{B}}^{1,0}}{2} \cdot \operatorname{deg} \Omega \frac{1}{B}\left(\log \Delta_{n c}\right)-\left(\sum_{i=2}^{q_{\bar{f}}-1} \xi_{i} \delta_{i}(\Upsilon)+\sum_{i=q_{\bar{f}}}^{[g / 2]} \eta_{i} \delta_{i}(\Upsilon)\right)
$$


where

$$
\begin{array}{ll}
\xi_{i}=-\frac{i(2 i+1)}{3} \cdot \beta_{1}+\beta_{i}, & \forall 2 \leq i \leq q_{\bar{f}}-1 ; \\
\eta_{i}=\frac{(2 i+1)(2 g+1-2 i)}{12(g+1)} \cdot \beta_{1}+\beta_{i}, & \forall q_{\bar{f}} \leq i \leq[g / 2] .
\end{array}
$$

Combining (4-17) with (4-4) and (4-16), it suffices to prove that $\xi_{i}$ 's and $\eta_{i}$ 's are all positive.

It is easy to see that $\xi_{i}>0$, since $\beta_{1} \leq 0$, and $\beta_{i} \geq \beta_{2}>0$ for $2 \leq i \leq q_{\bar{f}}-1$. Let $\Theta=(g-4)(2 g+1)-3(2 g-5) q_{\bar{f}}$. Then for $q_{\bar{f}} \leq i \leq[g / 2]$, one has

$$
\begin{aligned}
& \eta_{i}= \frac{1}{(g-1)(2 g+1)} \cdot\left(\left(2 g+1-3 q_{\bar{f}}\right)+\frac{\Theta}{12(g+1)}\right) \cdot i(g-i) \\
&-\frac{g-q_{\bar{f}}}{2 g+1}+\frac{\Theta \cdot(2 g+1)}{48(g-1)(2 g+1)(g+1)} \\
& \geq \eta_{q_{\bar{f}}}= \frac{g-q_{\bar{f}}}{48(g-1)(g+1)} \cdot\left(4 q_{\bar{f}}\left(13 g-21 q_{\bar{f}}+8\right)-50 g-51\right. \\
&\left.\quad+\frac{4\left(\left(q_{\bar{f}}-1\right)+\left(g-2 q_{\bar{f}}\right)(g-1)\right)}{g-q_{\bar{f}}}\right) \\
& \geq \frac{g-q_{\bar{f}}}{48(g-1)(g+1)} \cdot\left(4 q_{\bar{f}}\left(13 g-21 q_{\bar{f}}+8\right)-50 g-51\right) .
\end{aligned}
$$

Note that for $2 \leq q_{\bar{f}} \leq(g-1) / 2$, we have

$$
\begin{aligned}
& 4 q_{\bar{f}}\left(13 g-21 q_{\bar{f}}+8\right)-50 g-51 \\
\geq & \min \left\{4 \cdot 2 \cdot(13 g-21 \cdot 2+8), 4 \cdot \frac{g-1}{2} \cdot\left(13 g-21 \cdot \frac{g-1}{2}+8\right)\right\}-50 g-51 \\
> & 0, \quad \text { since } g>7 .
\end{aligned}
$$

Hence for $q_{\bar{f}} \leq i \leq[g / 2], \eta_{i}>0$. This completes the proof.

4.3. Proofs of the main results. Theorems $\mathrm{A}$ and $\mathrm{E}$ are immediate consequence of Theorems 1.3 and 1.4 and Theorem $\mathrm{D}$ follows from the definition (cf. Definition 3.3) and Theorem 4.6. Corollary $\mathrm{B}$ follows from Theorem $\mathrm{A}$ together with Lemma 2.13

Finally, we prove Corollary $\mathrm{C}$ as follows. Let $Z$ be as in Corollary C. Note that any Hecke translate of a Shimura curve of type I is still a Shimura curve of type I. Hence by Remark 2.7 and Definition 2.12, $Z$ contains a Shimura curve $C$ of type I. If moreover $Z \Subset \mathcal{T}_{g}$, then one can even assume $C \Subset \mathcal{T}_{g}$ (cf. Remark 2.7). Therefore our corollary follows from Theorem A

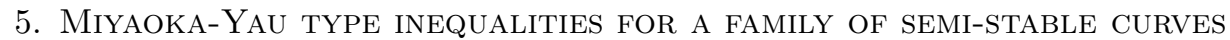

The section is aimed to prove a Miyaoka-Yau type inequality for families of semistable curves; as a consequence, we will complete the proofs of Theorems 4.1 and 4.4

Theorem 5.1. Let $\bar{f}: \bar{S} \rightarrow \bar{B}$ be a family of semi-stable curves of genus $g \geq 2$. For any $p \in \bar{B}$, let $F_{p}=f^{-1}(p)$ and

$$
\begin{aligned}
\Lambda^{\prime} & =\left\{p \in \bar{B} \mid \begin{array}{l}
F_{p} \text { is a hyperelliptic curve with compact Jacobian, and any } \\
\text { two irreducible irrational components of } F_{p} \text { do not intersect }
\end{array}\right\} ; \\
\Delta_{c t, b} & =\left\{p \in \Delta_{c t} \backslash \Lambda^{\prime} \mid \text { each irreducible component of } F_{p} \text { is of genus } 0 \text { or } 1\right\} ; \\
\Delta_{c t, u b} & =\Delta_{c t} \backslash\left\{\Lambda^{\prime} \cup \Delta_{c t, b}\right\} .
\end{aligned}
$$


Then

$$
\begin{aligned}
\omega \frac{2}{\bar{S} / \bar{B}} \leq & (2 g-2) \cdot \operatorname{deg}\left(\Omega \frac{1}{B}\left(\log \Delta_{n c}\right)\right)+\sum_{p \in \Delta_{c t} \cap \Lambda^{\prime}} \frac{3}{2} \cdot\left(l_{h}\left(F_{p}\right)+l_{1}\left(F_{p}\right)-1\right) \\
& +\left(2 g-2-\frac{g-1}{6}\right) \cdot\left|\Delta_{c t, b}\right|+\sum_{p \in \Delta_{c t, u b}}\left(3 l_{h}\left(F_{p}\right)+2 l_{1}\left(F_{p}\right)-3\right) .
\end{aligned}
$$

Moreover, if $\Delta_{n c} \neq \emptyset$ or $\Delta=\emptyset$, then the above inequality is strict.

We first prove in Section 5.1 Theorems 4.1 and 4.4 based on Theorem 5.1 which will be proved in Section 5.2 .

\subsection{Proof of Theorems 4.1 and 4.4 based on Theorem [5.1.}

Proof of Theorem 4.1. By Theorem 5.1, it suffices to prove

$$
\begin{aligned}
& \frac{3}{2} \cdot\left(l_{h}\left(F_{p}\right)+l_{1}\left(F_{p}\right)-1\right)<2 \delta_{1}\left(F_{p}\right)+3 \delta_{h}\left(F_{p}\right), \quad \forall p \in \Delta_{c t} \cap \Lambda^{\prime} ; \\
& 2 g-2-\frac{g-1}{6}<2 \delta_{1}\left(F_{p}\right)+3 \delta_{h}\left(F_{p}\right), \quad \forall p \in \Delta_{c t, b} ; \\
& 3 l_{h}\left(F_{p}\right)+2 l_{1}\left(F_{p}\right)-3 \leq 2 \delta_{1}\left(F_{p}\right)+3 \delta_{h}\left(F_{p}\right), \quad \forall p \in \Delta_{c t, u b} .
\end{aligned}
$$

Actually, (5-2) follows directly from (2-7). Similarly for (5-3) if one notes $l_{1}\left(F_{p}\right)=g$ and $l_{i}\left(F_{p}\right)=0$ for $i>1$ when $p \in \Delta_{c t, b}$. In order to prove (5-4), we first claim that for any $p \in \Delta_{c t, u b}$, we have

$$
l_{h}\left(F_{p}\right)-1 \leq \delta_{h}\left(F_{p}\right) .
$$

Indeed, let

$$
\mathcal{S}_{p}=\left\{\begin{array}{l|l}
q \in F_{p} & \begin{array}{l}
q \text { is a node of } F_{p}, \text { and each of the two connected components } \\
\text { of } F_{p} \backslash q \text { contains an irreducible component of genus } \geq 2
\end{array}
\end{array}\right\} .
$$

Since $F_{p}$ is connected, we get $l_{h}\left(F_{p}\right)-1 \leq\left|\mathcal{S}_{p}\right|$. It is clear that $\left|\mathcal{S}_{p}\right| \leq \delta_{h}\left(F_{p}\right)$. Hence (5-5) follows. Now again by (2-7), one gets for any $p \in \Delta_{c t, u b}$,

$$
\begin{aligned}
3 l_{h}\left(F_{p}\right)+2 l_{1}\left(F_{p}\right)-3 & =\left(l_{h}\left(F_{p}\right)-1\right)+2\left(l_{1}\left(F_{p}\right)+l_{h}\left(F_{p}\right)-1\right) \\
& \leq \delta_{h}\left(F_{p}\right)+2\left(\delta_{1}\left(F_{p}\right)+\delta_{h}\left(F_{p}\right)\right) \\
& =2 \delta_{1}\left(F_{p}\right)+3 \delta_{h}\left(F_{p}\right) .
\end{aligned}
$$

This completes the proof.

Proof of Theorem 4.4. Let $\bar{f}$ be as in Theorem 4.4. Then by the definition, it is easy to show that

$$
\begin{array}{ll}
2 g-2-\frac{g-1}{6} \leq 2 g-3=3 l_{h}\left(F_{p}\right)+2 l_{1}\left(F_{p}\right)-3, & \text { if } p \in \Delta_{c t, b} \text { and } g \geq 7 ; \\
\frac{3}{2} \cdot\left(l_{h}\left(F_{p}\right)+l_{1}\left(F_{p}\right)-1\right) \leq 3 l_{h}\left(F_{p}\right)+2 l_{1}\left(F_{p}\right)-3, & \text { if } p \in \Delta_{c t} \cap \Lambda^{\prime} \text { and } g \geq 3 .
\end{array}
$$

Thus by Theorem [5.1, it suffices to prove $\Lambda \subseteq \Lambda^{\prime}$. For this, it is enough to prove

Lemma 5.2. For $p \in \Lambda$, the fibre $F_{p}$ is hyperelliptic; if moreover $F_{p}$ is singular, then any two irreducible irrational components of $F_{p}$ do not intersect. 
To prove the above lemma, we may assume that $\Lambda \neq \emptyset$. Consider the following Cartesian diagram:

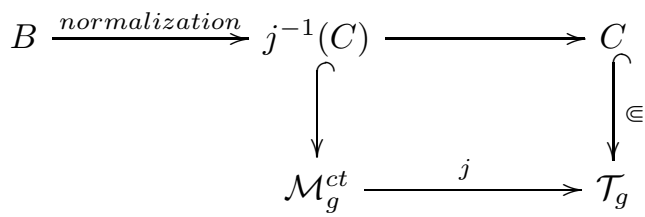

Note that the family $f: S \rightarrow B$ is obtained by pulling back the universe family over $\mathcal{M}_{g}^{c t}$ and resolving the singularities. Hence by Lemma A.1 there exists an involution $\sigma$ (resp. $\tau$ ) of $S$ (resp. B), such that $f \circ \sigma=\tau \circ f$ and the fixed locus of $\tau$ is $\operatorname{Fix}(\tau)=\Lambda$. Furthermore, for $p \in \Lambda$, the fibre $F_{p}$ is a hyperelliptic curve and the restricted involution

$$
\left.\sigma\right|_{F_{p}}: F_{p} \rightarrow F_{p}
$$

is the hyperelliptic involution of $F_{p}$. It remains to prove the last statement.

Assume that there are two irrational components $D_{1}$ and $D_{2}$ of $F_{p}$ with an intersection point $q$. Locally, we may assume that $D_{1}\left(\operatorname{resp} . D_{2}\right)$ is defined by $x=0$ (resp. $y=0)$, and $f$ is given by $t=f(x, y)=x y$, where $t(\operatorname{resp} .(x, y))$ is a local coordinate of $B$ (resp. $S$ ) around $p$ (resp. $q$ ).

Since the involution $\left.\sigma\right|_{F_{p}}$ is the hyperelliptic involution of $F_{p}, F_{p} /\left\langle\left.\sigma\right|_{F_{p}}\right\rangle$ is a (may be singular) rational curve. In particular, $\sigma$ keeps both $D_{1}$ and $D_{2}$ invariant, and $\left.\sigma\right|_{D_{1}}$ (resp. $\left.\sigma\right|_{D_{2}}$ ) is not the identity on $D_{1}$ (resp. $D_{2}$ ), since both $D_{1}$ and $D_{2}$ are irrational curves. The first implies that there exist non-zero functions $\xi(x, y)$ and $\eta(x, y)$ such that

$$
\sigma^{*}(x)=\xi x, \quad \sigma^{*}(y)=\eta y, \quad \text { with } \xi_{0}:=\xi(0,0) \neq 0 \text { and } \eta_{0}:=\eta(0,0) \neq 0 .
$$

Since $\sigma$ is an involution, we get that $\sigma^{*}\left(\sigma^{*}(x)\right)=x$ and $\sigma^{*}\left(\sigma^{*}(y)\right)=y$, from which it follows that $\xi_{0}^{2}=\eta_{0}^{2}=1$. Let

$$
\tilde{x}=x+\frac{\xi}{\xi_{0}} \cdot x, \quad \tilde{y}=y+\frac{\eta}{\eta_{0}} \cdot y, \quad \text { and } \quad \tilde{t}=\frac{\left(\xi_{0}+\xi\right)\left(\eta_{0}+\eta\right)}{\xi_{0} \eta_{0}} \cdot t
$$

It is easy to see that $\tilde{t}$ (resp. $(\tilde{x}, \tilde{y}))$ can be viewed as a local coordinate of $B$ (resp. $S$ ) around $p$ (resp. $q$ ). Moreover, $D_{1}$ (resp. $D_{2}$ ) is locally defined by $\tilde{x}=0$ (resp. $\tilde{y}=0), \bar{f}$ is given by $\tilde{t}=\tilde{x} \tilde{y}$, and

$$
\begin{aligned}
& \sigma^{*}(\tilde{x})=\sigma^{*}\left(x+\frac{1}{\xi_{0}} \sigma^{*}(x)\right)=\sigma^{*}(x)+\frac{1}{\xi_{0}} x=\xi_{0} \cdot \tilde{x} \\
& \sigma^{*}(\tilde{y})=\sigma^{*}\left(y+\frac{1}{\eta_{0}} \sigma^{*}(y)\right)=\sigma^{*}(y)+\frac{1}{\eta_{0}} y=\eta_{0} \cdot \tilde{y}
\end{aligned}
$$

As $\left.\sigma\right|_{D_{1}}$ (resp. $\left.\sigma\right|_{D_{2}}$ ) is not the identity on $D_{1}$ (resp. $D_{2}$ ), one gets that $\eta_{0} \neq 1$ (resp. $\xi_{0} \neq 1$ ), since $\left.y\right|_{D_{1}}$ (resp. $\left.x\right|_{D_{2}}$ ) is a local coordinate of $D_{1}$ (resp. $D_{2}$ ). Hence $\xi_{0}=\eta_{0}=-1$.

Note that $\tau \circ f=f \circ \sigma$. So

$$
f^{*} \tau^{*}(\tilde{t})=\sigma^{*} f^{*}(\tilde{t})=\sigma^{*}(\tilde{x} \tilde{y})=\tilde{\xi}_{0} \tilde{\eta}_{0} \cdot \tilde{x} \tilde{y}=\tilde{x} \tilde{y}=f^{*}(\tilde{t}) .
$$

Since $\bar{f}$ is surjective, one gets $\tau^{*}(\tilde{t})=\tilde{t}$, which implies that $\tau$ is the identity map of $B$ around $p$. It is a contradiction. This completes the proof of Lemma 5.2 and hence also Theorem 4.4 . 
5.2. Proof of Theorem 5.1. Our proof of Theorem5.1 is based on a generalized Miyaoka-Yau's inequality (cf. Theorem [5.3), by choosing a suitable base change and suitable components contained in singular fibres (but not the entire singular fibres).

Recall from 41 the generalized Miyaoka-Yau's theorem. Let $X_{x}$ be the germ of a quotient singularity of $\left(\mathbb{C}^{2} / G_{x}\right)_{0}$ (in the analytic sense), where $G_{x}$ is a finite subgroup of $\mathrm{GL}(2, \mathbb{C})$ with the origin 0 being its unique fixed point. Let $X_{E}$ be the minimal resolution of $X_{x}$ and $E$ the exceptional divisor (= the inverse image of $x$ ). Let

$$
v(x) \triangleq \chi_{\mathrm{top}}(E)-\frac{1}{\left|G_{x}\right|} .
$$

Theorem 5.3 (Miyaoka [41, Corollary 1.3]). Let $X^{\#}$ be a projective surface with only rational double singularities, and $\mathcal{I}$ the singular locus of $X^{\#}$. Let $D$ be a reduced normal crossing curve which lies on the smooth part of $X^{\#}$. Let $X$ be the minimal resolution of $X^{\#}$. Assume that $\mathcal{O}_{X}\left(K_{X}\right)$ is numerically effective. Then

$$
\sum_{x \in \mathcal{I}} v(x) \leq \chi_{\text {top }}(X)-\chi_{\text {top }}(D)-\frac{1}{3}\left(\omega_{X}+D\right)^{2} .
$$

Note that for a singularity $x$ of type $A_{k}$, the invariant $v(x)$ defined in (5-6) is equal to $(k+1)-\frac{1}{k+1}$. Therefore we get

Theorem 5.4. Let conditions be the same as that of Theorem 5.3. Assume that each point $x \in \mathcal{I}$ is a quotient singularities of type $A_{k_{x}}, X$ is minimal and of general type. Then

$$
\sum_{x \in \mathcal{I}}\left(3\left(k_{x}+1\right)-\frac{3}{k_{x}+1}\right) \leq 3\left(\chi_{\mathrm{top}}(X)-\chi_{\mathrm{top}}(D)\right)-\left(\omega_{X}+D\right)^{2} .
$$

Proof of Theorem 5.1. Let

$$
\begin{aligned}
& E_{p}=\left\{\sum_{j} E_{p, j} \mid E_{p, j} \subseteq F_{p} \text { is a }(-2) \text {-curve }\right\}, \quad \forall p \in \Delta \backslash \Delta_{c t, u b} ; \\
& D_{p}=\left\{\sum_{j} D_{p, j} \mid D_{p, j} \subseteq F_{p} \text { with genus } g\left(D_{p, j}\right)=0 \text { or } 1\right\}, \forall p \in \Delta_{c t, u b} .
\end{aligned}
$$

Let $\bar{S} \rightarrow \bar{S}^{\#}$ be the contraction of

$$
\sum_{p \in \Delta \backslash \Delta_{c t, u b}} E_{p} \subseteq \bar{S}
$$

and $f^{\#}: \bar{S}^{\#} \rightarrow \bar{B}$ the induced morphism. It is clear that for any $p \in \Delta_{c t, u b}$, the image of $D_{p}$ on $\bar{S}^{\#}$ lies on the smooth part of $\bar{S}^{\#}$, which we still denote by $D_{p}$. For any singular point $q$ of $\bar{S}^{\#},\left(\bar{S}^{\#}, q\right)$ is a rational double point of type $A_{\lambda_{q}}$, here $\lambda_{q}$ is the number of $(-2)$-curves in $\bar{S}$ over $q$. For convenience, we also denote by $q$ the singular point of the fibres on the smooth part of $\bar{S}^{\#}$, in which case, $\lambda_{q}=0$. So a singular point $\left(\bar{S}^{\#}, q\right)$ of type $A_{0}$ is understood as a node of the fibres but a smooth point of $\bar{S}^{\#}$. For $p \in \bar{B}$, let $F_{p}^{\#}$ be the image of $F_{p}$ on $\bar{S}^{\#}$. Then it is clear that

$$
\delta\left(F_{p}\right)=\sum_{q \in F_{p}^{\#}}\left(\lambda_{q}+1\right), \quad \forall p \in \Delta \backslash \Delta_{c t, u b} .
$$


Let $\phi: \widetilde{B} \rightarrow \bar{B}$ be a cover of $\bar{B}$ such that $\phi$ is branched uniformly over $\Delta_{n c}$ (resp. $\Delta_{c t, b}$ ) with ramification index equaling to $e_{n c}$ (resp. $e_{c t, b}$ ). Such a cover exists. Indeed, by the Kodaira-Parshin construction (cf. 70] and 64]), one can first construct a cover $\phi^{\prime}: B^{\prime} \rightarrow \bar{B}$ branched uniformly over $\Delta_{n c}$ with ramification index equaling to $e_{n c}$; and then take a cover $\widetilde{B} \rightarrow B^{\prime}$ branched uniformly over $\left(\phi^{\prime}\right)^{-1}\left(\Delta_{c t, b}\right)$ with ramification index equaling to $e_{c t, b}$. Then the composition $\phi$ : $\widetilde{B} \rightarrow \bar{B}$ satisfies our requirements. Let $\operatorname{deg} \phi=d$. Then according to Hurwitz formula, one gets

$$
2(g(\widetilde{B})-1)=d \cdot\left(2(g(\bar{B})-1)+\frac{e_{n c}-1}{e_{n c}} \cdot\left|\Delta_{n c}\right|+\frac{e_{c t, b}-1}{e_{c t, b}} \cdot\left|\Delta_{c t, b}\right|\right) .
$$

Let $\widetilde{S}^{\#}=\widetilde{B} \times{ }_{B} \bar{S}^{\#}$ be the fibre-product, and $\widetilde{S} \rightarrow \widetilde{S}^{\#}$ the minimal resolution of singularities. We have the following commutative diagram:

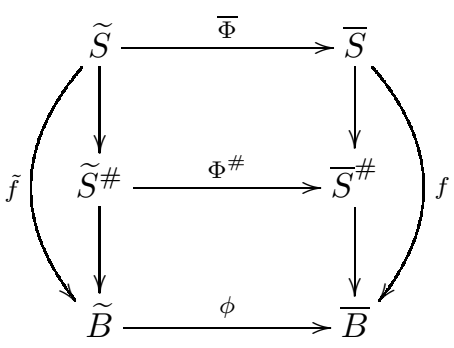

For $p \in \Delta_{c t} \cap \Lambda^{\prime}$ (resp. $p \in \Delta_{n c}$, resp. $p \in \Delta_{c t, b}$ ), the inverse image of a singular point $\left(\bar{S}^{\#}, q\right)$ of type $A_{\lambda_{q}}$ with $q \in F_{p}^{\#}$ is $d$ (resp. $\frac{d}{e_{n c}}$, resp. $\frac{d}{e_{c t, b}}$ ) singular points of type $A_{\lambda_{q}}$ (resp. $A_{\left(\lambda_{q}+1\right) \cdot e_{n c}-1}$, resp. $\left.A_{\left(\lambda_{q}+1\right) \cdot e_{c t, b}-1}\right)$ in $\widetilde{S}^{\#}$. Let

$$
D=\sum_{p \in \Delta_{c t, u b}}\left(\Phi^{\#}\right)^{-1}\left(D_{p}\right)
$$

Since $\phi$ is unbranched over $\Delta_{c t, u b}, D$ lies on the smooth part of $\widetilde{S}^{\#}$ and

$$
3 \chi_{\mathrm{top}}(D)+2 \omega_{\widetilde{S}} \cdot D+D^{2}=d \cdot \sum_{p \in \Delta_{c, u b}}\left(3 \chi_{\mathrm{top}}\left(D_{p}\right)+2 \omega_{\bar{S}} \cdot D_{p}+D_{p}^{2}\right)
$$

Because $\bar{f}$ is semi-stable, $\tilde{f}: \widetilde{S} \rightarrow \widetilde{B}$ is also semi-stable, and

$$
\delta_{\tilde{f}}=d \cdot \delta_{\bar{f}}, \quad \omega_{\widetilde{S} / \widetilde{B}}^{2}=d \cdot \omega \frac{2}{\bar{S} / \bar{B}} .
$$

It is not difficult to see that $\widetilde{S}$ is minimal and of general type if $g(\widetilde{B}) \geq 1$, which is satisfied when $d$ is large enough. Hence applying Theorem 5.4 to the case by 
setting $X^{\#}=\widetilde{S}^{\#}, X=\widetilde{S}$, and $D$ as above, we get

$$
\begin{aligned}
& 3 d \cdot \sum_{\substack{q \in F_{p}^{\#} \\
p \in \Delta_{c t} \cap \Lambda^{\prime}}}\left(\left(\lambda_{q}+1\right)-\frac{1}{\lambda_{q}+1}\right)+\frac{3 d}{e_{n c}} \cdot \sum_{\substack{q \in F_{p}^{\#} \\
p \in \Delta_{n c}}}\left(\left(\lambda_{q}+1\right) e_{n c}-\frac{1}{\left(\lambda_{q}+1\right) e_{n c}}\right) \\
& +\frac{3 d}{e_{c t, b}} \cdot \sum_{\substack{q \in F_{p}^{\#} \\
p \in \Delta_{c t, b}}}\left(\left(\lambda_{q}+1\right) \cdot e_{c t, b}-\frac{1}{\left(\lambda_{q}+1\right) \cdot e_{c t, b}}\right) \\
& \leq 3\left(\chi_{\mathrm{top}}(\widetilde{S})-\chi_{\mathrm{top}}(D)\right)-\left(\omega_{\widetilde{S}}+D\right)^{2} \\
& =d \cdot\left(3 \delta_{\bar{f}}-\omega_{\bar{S}}^{2} / \bar{B}\right)-d \cdot \sum_{p \in \Delta_{c t, u b}}\left(3 \chi_{\mathrm{top}}\left(D_{p}\right)+2 \omega_{\bar{S}} \cdot D_{p}+D_{p}^{2}\right) \\
& \quad+d \cdot(2 g-2)\left(2(g(\bar{B})-1)+\frac{e_{n c}-1}{e_{n c}} \cdot\left|\Delta_{n c}\right|+\frac{e_{c, b}-1}{e_{c t, b}} \cdot\left|\Delta_{c t, b}\right|\right) .
\end{aligned}
$$

We use (2-1), (5-11), (5-12) and (5-13) in the last step above. By (2-5) and (5-17), we have

$$
\delta_{\bar{f}}=\sum_{\substack{q \in F_{p}^{\#} \\ p \in \Delta_{c t} \cap \Lambda^{\prime}}}\left(\lambda_{q}+1\right)+\sum_{\substack{q \in F_{p}^{\#} \\ p \in \Delta_{n c}}}\left(\lambda_{q}+1\right)+\sum_{\substack{q \in F_{p}^{\#} \\ p \in \Delta_{c t, b}}}\left(\lambda_{q}+1\right)+\sum_{p \in \Delta_{c t, u b}} \delta\left(F_{p}\right) .
$$

Combining (5-14) with (5-15), one gets

$$
\begin{aligned}
\omega \frac{2}{S} / \bar{B} \leq & (2 g-2) \cdot\left(2(g(\bar{B})-1)+\left|\Delta_{n c}\right|+\left|\Delta_{c t, b}\right|\right)+\sum_{\substack{q \in F_{p}^{\#} \\
p \in \Delta_{c t} \cap \Lambda^{\prime}}} \frac{3}{\lambda_{q}+1} \\
& +\left(\sum_{\substack{q \in F_{p}^{\#} \\
p \in \Delta_{n c}}} \frac{1}{\left(\lambda_{q}+1\right)}\right) \cdot \frac{3}{e_{n c}^{2}}-\frac{(2 g-2) \cdot\left|\Delta_{n c}\right|}{e_{n c}} \\
& +\sum_{p \in \Delta_{c t, b}}\left(\sum_{q \in F_{p}^{\#}} \frac{1}{\left(\lambda_{q}+1\right)} \cdot \frac{3}{e_{c t, b}^{2}}-\frac{2 g-2}{e_{c t, b}}\right) \\
& +\sum_{p \in \Delta_{c t, u b}}\left(3 \delta\left(F_{p}\right)-\left(3 \chi_{\mathrm{top}}\left(D_{p}\right)+2 \omega_{\bar{S}} \cdot D_{p}+D_{p}^{2}\right)\right)
\end{aligned}
$$

We have the following claim, whose proof are given at the end of the section.

Claim 5.5. (i). For each $p \in \Delta_{c t} \cap \Lambda^{\prime}$,

$$
\sum_{q \in F_{p}^{\#}} \frac{1}{\lambda_{q}+1} \leq \frac{1}{2}\left(l_{h}\left(F_{p}\right)+l_{1}\left(F_{p}\right)-1\right)
$$

(ii). For each $p \in \Delta_{c t, b}$,

$$
\sum_{q \in F_{p}^{\#}} \frac{1}{\lambda_{q}+1}<2 g-2
$$

(iii). For each $p \in \Delta_{c t, u b}$,

$$
3 \delta\left(F_{p}\right)-\left(3 \chi_{\text {top }}\left(D_{p}\right)+2 \omega_{\bar{S}} \cdot D_{p}+D_{p}^{2}\right) \leq 3 l_{h}\left(F_{p}\right)+2 l_{1}\left(F_{p}\right)-3 .
$$


By taking $e_{c t, b}=6$ and $e_{n c} \rightarrow \infty$ in (5-16), we get the required inequality (5-1). If $\Delta_{n c} \neq \emptyset$, then letting $e_{n c}$ be large enough, one has

$$
\left(\sum_{\substack{q \in F_{p}^{\#} \\ p \in \Delta_{n c}}} \frac{1}{\left(\lambda_{q}+1\right)}\right) \cdot \frac{3}{e_{n c}^{2}}-\frac{(2 g-2) \cdot\left|\Delta_{n c}\right|}{e_{n c}}<0 .
$$

Hence if $\Delta_{n c} \neq \emptyset$, then the inequality (5-1) is strict by letting $e_{c t, b}=6$ and $e_{n c}$ be large enough. Finally, if $\Delta=\emptyset$, then $\bar{f}$ is a Kodaira family, and

$$
\operatorname{deg}\left(\Omega \frac{1}{B}\left(\log \Delta_{n c}\right)\right)=2 g(\bar{B})-2 .
$$

So by [36, Corollary 0.6], (5-1) is also strict in the case. The proof is complete.

Remarks 5.6. (i). If (4-1) is indeed an equality, i.e.,

$$
\omega \frac{2}{\bar{S} / \bar{B}}=(2 g-2) \cdot \operatorname{deg}\left(\Omega \frac{1}{B}\left(\log \Delta_{n c}\right)\right)+2 \delta_{1}\left(\Upsilon_{c t}\right)+3 \delta_{h}\left(\Upsilon_{c t}\right),
$$

then $\Delta_{n c}=\emptyset ; \Delta_{c t} \cap \Lambda^{\prime}=\emptyset$ by (5-2); $\Delta_{c t, b}=\emptyset$ by (5-3); and $l_{0}\left(F_{p}\right)=0$ for $p \in \Delta_{c t, u b}$ by (5-4) and its proof. In particular, $D_{p}$ contains at most elliptic curves for $p \in \Delta_{c t}=\Delta_{c t, u b}$. Hence (5-20) is equivalent to

$$
c_{1}^{2}\left(\Omega \frac{1}{S}\left(\log \left(\sum_{p \in \Delta_{c t}} D_{p}\right)\right)\right)=3 c_{2}\left(\Omega \frac{1}{S}\left(\log \left(\sum_{p \in \Delta_{c t}} D_{p}\right)\right)\right) .
$$

It follows that $\bar{S} \backslash\left(\bigcup_{p \in \Delta_{c t}} D_{p}\right)$ is a ball quotient by [30] or [43]. See Example 8.2 for such an example.

(ii). If one applies Theorem 5.4 directly on the surface $\bar{S}$ without using base change technique, then one gets

$$
\begin{aligned}
\omega_{\bar{S} / \bar{B}}^{2} \leq & (2 g-2) \cdot \operatorname{deg}\left(\Omega \frac{1}{B}\left(\log \Delta_{n c}\right)\right)+\sum_{p \in \Delta_{c t} \cap \Lambda^{\prime}} \frac{3}{2} \cdot\left(l_{h}\left(F_{p}\right)+l_{1}\left(F_{p}\right)-1\right) \\
& +(2 g-2) \cdot\left|\Delta_{c t, b}\right|+\sum_{p \in \Delta_{c t, u b}}\left(3 l_{h}\left(F_{p}\right)+2 l_{1}\left(F_{p}\right)-3\right) .
\end{aligned}
$$

This is enough to imply (4-1). However, we do not know when (4-1) becomes strict; and we cannot derive (4-5) from (5-21) due to the possible existence of $\Delta_{c t, b}$. Recently, Peters has gotten in 57 a simplified proof of the strictness of (4-1) if $\Delta_{n c} \neq \emptyset$ by using Cheng-Yau's technique instead of the base change technique.

In the rest part of the section, we prove Claim 5.5. First we prove an easy lemma.

Lemma 5.7. Assume that $F^{\#}$ is a stable hyperelliptic curve with compact Jacobian. Then $F^{\#}$ has no rational component.

Proof. By [9, p. 467], $F^{\#}$ has a semi-stable model $F$ which is an admissible double cover $\psi: F \rightarrow \Gamma$ over a stable $(2 g+2)$-pointed nodal curve $\Gamma$ of arithmetic genus zero.

We claim that the index of every singular point is odd. Otherwise, assume that there exists a singular point $p \in \Gamma$ with even index $\alpha$. Then $p$ is not a branched point of $\psi$ and its inverse image consists of two singular points of type 0 , which contradicts with the fact that $F$ has compact Jacobian. As a direct consequence of all indices being odd, we obtain that all singular points of $\Gamma$ are branched, and hence the pre-image of any irreducible component of $\Gamma$ in $F$ is still irreducible.

Let $D \subseteq F$ be an irreducible component and $D^{\prime}=\psi(D) \subseteq \Gamma$. Set

$$
\Sigma^{\prime}=\left\{x \in D^{\prime} \mid x \text { is a marked or singular point of } \Gamma\right\} .
$$


As $\Gamma$ is stable, $\left|\Sigma^{\prime}\right| \geq 3$. From the above discussion, the restricted map

$$
\left.\psi\right|_{D}: D \longrightarrow D^{\prime}
$$

is a double cover branched exactly over $\Sigma^{\prime}$. Hence

$$
2 g(D)-2=2\left(2 g\left(D^{\prime}\right)-2\right)+\left|\Sigma^{\prime}\right| \geq-1,
$$

which implies that $g(D) \geq 1$. Therefore, $F$ and thus $F^{\#}$ contain no rational component.

Proof of Claim 5.5. Let $l\left(F_{p}^{\#}\right)$ (resp. $\left.v\left(F_{p}^{\#}\right)\right)$ be the number of irreducible components (resp. nodes) of $F_{p}^{\#}$. Then it is clear that

$$
v\left(F_{p}^{\#}\right)=l\left(F_{p}^{\#}\right)-1, \quad \forall p \in \Delta_{c t} .
$$

(i). Since $p \in \Lambda^{\prime}, F_{p}$ is a hyperelliptic curve. Hence by Lemma 5.7, $F_{p}^{\#}$ contains no rational components. So

$$
l\left(F_{p}^{\#}\right)=l_{h}\left(F_{p}\right)+l_{1}\left(F_{p}\right) .
$$

Now by the definition of $\Delta_{c t} \cap \Lambda^{\prime}$, each node of $F_{p}^{\#}$ is a singular point of $\bar{S}^{\#}$, i.e.,

$$
\lambda_{q} \geq 1, \quad \text { for any node } q \in F_{p}^{\#} \text { and } p \in \Delta_{c t} \cap \Lambda^{\prime} .
$$

Thus

$$
\sum_{q \in F_{p}^{\#}} \frac{1}{\lambda_{q}+1} \leq \frac{1}{2} v\left(F_{p}^{\#}\right)=\frac{1}{2}\left(l_{h}\left(F_{p}\right)+l_{1}\left(F_{p}\right)-1\right), \quad \forall p \in \Delta_{c t} \cap \Lambda^{\prime} .
$$

(ii). Note that $F_{p}^{\#}$ is the stable model of $F_{p}$ for $\in \Delta_{c t, b}$. Hence the inverse image $C \subseteq F_{p}$ of any component $C^{\#} \subseteq F_{p}^{\#}$ has positive intersection with $\omega_{\bar{S}}$, i.e., $\omega_{\bar{S}} \cdot C \geq 1$. Since $\omega_{\bar{S}} \cdot F_{p}=2 g-2$, one has

$$
l\left(F^{\#}\right) \leq 2 g-2 .
$$

Therefore

$$
\sum_{q \in F_{p}^{\#}} \frac{1}{\lambda_{q}+1} \leq v\left(F_{p}^{\#}\right)=l\left(F_{p}^{\#}\right)-1<2 g-2 .
$$

(iii). As $F_{p}$ has a compact Jacobian for $p \in \Delta_{c t, u b} \subseteq \Delta_{c t}$,

$$
3 \delta\left(F_{p}\right)=3\left(l_{0}\left(F_{p}\right)+l_{1}\left(F_{p}\right)+l_{h}\left(F_{p}\right)-1\right) .
$$

So it suffices to prove that

$$
3 \chi_{\text {top }}\left(D_{p}\right)+2 \omega_{\bar{S}} \cdot D_{p}+D_{p}^{2} \geq 3 l_{0}\left(F_{p}\right)+l_{1}\left(F_{p}\right)
$$

Let $l_{0}\left(D_{p}\right)$ and $l_{1}\left(D_{p}\right)$ be the number of irreducible components contained in $D_{p}$ of genus zero and one respectively. Since $p \in \Delta_{c t, u b}$, by the definition of $D_{p}$ (cf. (5-9)), we get

$$
l_{0}\left(D_{p}\right)=l_{0}\left(F_{p}\right), \quad l_{1}\left(D_{p}\right)=l_{1}\left(F_{p}\right) .
$$

Let $C_{p} \subseteq D_{p}$ be a connected component, and $l_{0}\left(C_{p}\right)$ and $l_{1}\left(C_{p}\right)$ be the number of irreducible components contained in $C_{p}$ of genus zero and one respectively. By $(5-23)$, in order to prove (5-22), it suffices to prove for each connected component $C_{p}$,

$$
3 \chi_{\text {top }}\left(C_{p}\right)+2 \omega_{\bar{S}} \cdot C_{p}+C_{p}^{2} \geq 3 l_{0}\left(C_{p}\right)+l_{1}\left(C_{p}\right) .
$$

For this purpose, note that $C_{p}^{2}<0$, since $C_{p} \subseteq D_{p} \varsubsetneqq F_{p}$ by the definition of $\Delta_{c t, u b}$. So

$$
3 \chi_{\text {top }}\left(C_{p}\right)+2 \omega_{\bar{S}} \cdot C_{p}+C_{p}^{2}=3 l_{0}\left(C_{p}\right)+l_{1}\left(C_{p}\right)-1-C_{p}^{2} \geq 3 l_{0}\left(C_{p}\right)+l_{1}\left(C_{p}\right) .
$$

The proof is complete. 


\section{Sharp SLOPE INEQUALities FORA FAMily of SEMI-STABle CURVES}

6.1. Proof of Theorem 4.2, The inequality (4-2) follows from Moriwaki's sharp slope inequality (cf. [49, Theorem D]), which together with (2-2) and (2-5) implies that

$$
\begin{aligned}
(8 g+4) \operatorname{deg} \bar{f}_{*} \omega_{\bar{S} / \bar{B}} & \geq g \delta_{0}(\Upsilon)+\sum_{i=1}^{[g / 2]} 4 i(g-i) \delta_{i}(\Upsilon) \\
& =g\left(12 \operatorname{deg} \bar{f}_{*} \omega_{\bar{S} / \bar{B}}-\omega_{\bar{S} / \bar{B}}^{2}\right)+\sum_{i=1}^{[g / 2]}(4 i(g-i)-g) \delta_{i}(\Upsilon) \\
& \geq g\left(12 \operatorname{deg} \bar{f}_{*} \omega_{\bar{S}} / \bar{B}-\omega_{\bar{S} / \bar{B}}^{2}\right)+(3 g-4) \delta_{1}(\Upsilon)+(7 g-16) \delta_{h}(\Upsilon)
\end{aligned}
$$

By rearrangement, we obtain (4-2).

6.2. Proof of Theorem 4.3. By assumption, $\bar{f}: \bar{S} \rightarrow \bar{B}$ is a non-isotrivial semistable family of hyperelliptic curves of genus $g \geq 2$ with relative irregularity $q_{\bar{f}}=$ $q(\bar{S})-g(\bar{B})$. Recall from [9, Proposition 4.7] a useful formula given by CornalbaHarris:

$$
\begin{aligned}
\operatorname{deg} \bar{f}_{*} \omega_{\bar{S} / \bar{B}}= & \frac{g}{4(2 g+1)} \xi_{0}(\Upsilon) \\
& +\sum_{i=1}^{[g / 2]} \frac{i(g-i)}{2 g+1} \delta_{i}(\Upsilon)+\sum_{j=1}^{[(g-1) / 2]} \frac{(j+1)(g-j)}{2(2 g+1)} \xi_{j}(\Upsilon) .
\end{aligned}
$$

The proof of Theorem 4.3 is given in Section 6.2.2. If $q_{\bar{f}}=0$, it follows directly from Noether's formula and (6-1). If $q_{\bar{f}}>0$, we first prove a relation among the invariants $\delta_{i}(\Upsilon)$ 's and $\xi_{j}(\Upsilon)$ 's in Proposition 6.1. based on the observation that the double cover induced by the hyperelliptic involution is fibred and the technique of Cornalba-Harris 9]. Then together with (6-1), we complete the proof.

6.2.1. Hyperelliptic family with positive relative irregularity. The main purpose of this subsection is to prove the following technical proposition.

Proposition 6.1. Let $\bar{f}: \bar{S} \rightarrow \bar{B}$ be the same as in Theorem 4.3. If $q_{\bar{f}}>0$, then

$$
\begin{aligned}
& \sum_{i=q_{\bar{f}}}^{[g / 2]} \frac{(2 i+1)(2 g+1-2 i)}{g+1} \delta_{i}(\Upsilon)+\sum_{j=q_{\bar{f}}}^{[(g-1) / 2]} \frac{2(j+1)(g-j)}{g+1} \xi_{j}(\Upsilon) \\
\geq & \xi_{0}(\Upsilon)+\sum_{i=1}^{q_{\bar{f}}-1} 4 i(2 i+1) \delta_{i}(\Upsilon)+\sum_{j=1}^{q_{\bar{f}}-1} 2(j+1)(2 j+1) \xi_{j}(\Upsilon) .
\end{aligned}
$$

As mentioned before, the key observation is that a double cover $\tilde{\pi}: \widetilde{S} \rightarrow \tilde{Y}$ of smooth surfaces is fibred, where $\tilde{\pi}$ is obtained by resolving the singular points of a double cover $\pi: \bar{S} \rightarrow \bar{Y}$ over a ruled surface $\bar{Y}$, while $\pi$ is induced by the hyperelliptic involution. Let $\widetilde{R} \subseteq \widetilde{Y}$ be the smooth branched divisor of $\tilde{\pi}, \tilde{f}: \widetilde{S} \rightarrow \bar{B}$ and $\tilde{h}: \widetilde{Y} \rightarrow \bar{B}$ be composite morphisms fitting into the following diagram. 


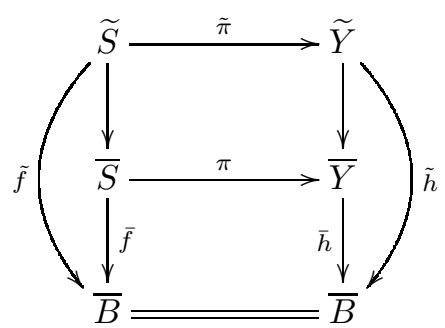

FiguRe 6.2-1. Hyperelliptic involution.

Definition 6.2 (28]). A double cover $\pi: X \rightarrow X^{\prime}$ of smooth projective surfaces with branched divisor $R^{\prime} \subseteq X^{\prime}$ is called fibred if there exists a double cover $\pi^{\prime}$ : $D \rightarrow D^{\prime}$ of smooth projective curves, morphisms $p: X \rightarrow D$ and $p^{\prime}: X^{\prime} \rightarrow D^{\prime}$ with connected fibres, such that the diagram

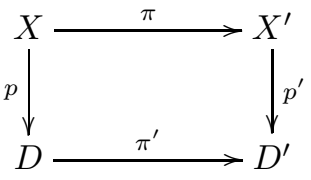

is commutative, $R^{\prime}$ is contained in the fibres of $p^{\prime}$, and

$$
q(X)-q\left(X^{\prime}\right):=\operatorname{dim} H^{0}\left(X, \Omega_{X}^{1}\right)-\operatorname{dim} H^{0}\left(X^{\prime}, \Omega_{X^{\prime}}^{1}\right)=g(D)-g\left(D^{\prime}\right) .
$$

The next theorem is proven in [28. For readers' convenience, we reprove it here.

Theorem 6.3 ([28, Theorem 1]). Let $\pi: X \rightarrow X^{\prime}$ be a double cover between smooth surfaces with smooth branched divisor $R^{\prime} \subseteq X^{\prime}$. Assume that $p_{g}\left(X^{\prime}\right):=$ $\operatorname{dim} H^{0}\left(X^{\prime}, \Omega_{X^{\prime}}^{2}\right)=0$ and $q(X)>q\left(X^{\prime}\right)$. Then $\pi: X \rightarrow X^{\prime}$ is fibred.

Proof. Note that the Galois group $\operatorname{Gal}\left(X / X^{\prime}\right) \cong \mathbb{Z}_{2}$ has a natural action on $H^{0}\left(X, \Omega_{X}^{1}\right)$. Let

be the eigenspace decomposition. Then

$$
H^{0}\left(X, \Omega_{X}^{1}\right)=H^{0}\left(X, \Omega_{X}^{1}\right)_{1} \oplus H^{0}\left(X, \Omega_{X}^{1}\right)_{-1}
$$

$$
H^{0}\left(X, \Omega_{X}^{1}\right)_{1}=\pi^{*} H^{0}\left(X^{\prime}, \Omega_{X^{\prime}}^{1}\right), \quad k \triangleq \operatorname{dim} H^{0}\left(X, \Omega_{X}^{1}\right)_{-1}=q(X)-q\left(X^{\prime}\right)>0 .
$$

We show there exists a morphism $p: X \rightarrow D$ to a curve $D$ with connected fibres and a subspace $W_{D} \subseteq H^{0}\left(D, \Omega_{D}^{1}\right)$, such that

$$
H^{0}\left(X, \Omega_{X}^{1}\right)_{-1}=p^{*}\left(W_{D}\right) .
$$

If $k=1$, let $A_{X}$ (resp. $A_{X^{\prime}}$ ) be the Albanese variety of $X$ (resp. $X^{\prime}$ ). Then we have a surjective homomorphism $A_{X} \rightarrow A_{X^{\prime}}$ with $\operatorname{dim} A_{X}-\operatorname{dim} A_{X^{\prime}}=k=1$. Hence there exists an elliptic curve $D$ with an isogeny (cf. [52]) $A_{X} \rightarrow A_{X}^{\prime} \times D$. By construction,

$$
p_{0}: X \longrightarrow A_{X} \longrightarrow A_{X}^{\prime} \times D \longrightarrow D
$$

is surjective. Then the desired morphism $p$ with connected fibres follows from the Stein factorization of $p_{0}$ (cf. [24, §III-11]), so does (6-3).

If $k \geq 2$, let $\omega, \omega^{\prime} \in H^{0}\left(X, \Omega_{X}^{1}\right)_{-1}$, then

$$
\omega \wedge \omega^{\prime} \in \wedge^{2} H^{0}\left(X, \Omega_{X}^{1}\right)_{-1} \subseteq H^{0}\left(X, \Omega_{X}^{2}\right)
$$

is invariant under the action of $\operatorname{Gal}\left(X / X^{\prime}\right)$ and thus belongs to $\pi^{*}\left(H^{0}\left(X^{\prime}, \Omega_{X^{\prime}}^{2}\right)\right)$, which is zero by our assumption. By [4, Proposition 5.1], there exists a morphism $p: X \rightarrow D$ with connected fibres such that (6-3) holds.

Note that (6-3) implies that $p: X \rightarrow D$ is unique. In particular, the Galois action $\operatorname{Gal}\left(X / X^{\prime}\right)$ on $X$ induces a group action $\mathbb{Z}_{2}$ on $D$. Let $D^{\prime}=D / \mathbb{Z}_{2}$, and 
$\pi^{\prime}: D \rightarrow D^{\prime}$ be the natural morphism. Then by construction, there exists a morphism $p^{\prime}: X^{\prime} \rightarrow D^{\prime}$ such that $p^{\prime} \circ \pi=\pi^{\prime} \circ p$.

Let $1 \neq \sigma \in \operatorname{Gal}\left(X / X^{\prime}\right)$. Then the fixed locus $\operatorname{Fix}(\sigma)$ of $\sigma$ is clearly contained in the fibres of $p$. So $R^{\prime}=\pi(\operatorname{Fix}(\sigma))$ is contained in the fibres of $p^{\prime}$. By (6-3), one sees that the eigenspace decomposition of $H^{0}\left(D, \Omega_{D}^{1}\right)$ with respect to the action of $\mathbb{Z}_{2}$ is

with

$$
H^{0}\left(D, \Omega_{D}^{1}\right)=\left(\pi^{\prime}\right)^{*} H^{0}\left(D^{\prime}, \Omega_{D^{\prime}}^{1}\right) \oplus H^{0}\left(D, \Omega_{D}^{1}\right)_{-1},
$$

$$
H^{0}\left(X, \Omega_{X}^{1}\right)_{-1}=p^{*} H^{0}\left(D, \Omega_{D}^{1}\right)_{-1} .
$$

So $q(X)-q\left(X^{\prime}\right)=k=g(D)-g\left(D^{\prime}\right)$. The proof is complete.

Come back to our case $\tilde{\pi}: \widetilde{S} \rightarrow \widetilde{Y}$. Note that $q(\widetilde{S})=q(\bar{S})$ and $q(\tilde{Y})=g(\bar{B})$. If $q_{\bar{f}}=q(\bar{S})-g(\bar{B})>0$, it follows that $q(\widetilde{S})>q(\widetilde{Y})$. As $\tilde{Y}$ is a ruled surface, the geometric genus $p_{g}(\widetilde{Y})=0$. Hence by Theorem 6.3 above, we get

Proposition 6.4. The double cover $\tilde{\pi}: \widetilde{S} \rightarrow \widetilde{Y}$ is fibred, i.e., there exist a double cover $\pi^{\prime}: \bar{B}^{\prime} \rightarrow \bar{D}^{\prime}$ of smooth projective curves, and morphisms $\tilde{f}^{\prime}: \widetilde{S} \rightarrow \bar{B}^{\prime}$ and $\tilde{h}^{\prime}: \tilde{Y} \rightarrow \bar{D}^{\prime}$ with connected fibres, such that the diagram

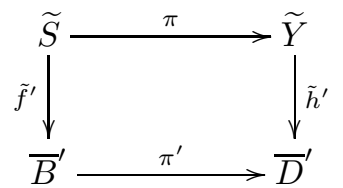

is commutative, $\widetilde{R}$ is contained in the fibres of $\tilde{h}^{\prime}$ and

$$
q_{\bar{f}}=q(\widetilde{S})-q(\widetilde{Y})=g\left(\bar{B}^{\prime}\right)-g\left(\bar{D}^{\prime}\right) .
$$

Since $\tilde{Y}$ is a ruled surface, it is easy to see that $\bar{D}^{\prime} \cong \mathbb{P}^{1}$. So by (6-4), $g\left(\bar{B}^{\prime}\right)=$ $q_{\bar{f}}>0$, which implies that $\tilde{f}^{\prime}$ factors through $\bar{S}$ as

$$
\tilde{f}^{\prime}: \widetilde{S} \longrightarrow \bar{S} \stackrel{\bar{f}^{\prime}}{\longrightarrow} B^{\prime} .
$$

Proposition 6.5. Let $F$ (resp. $F^{\prime}$ ) be any fibre of $\bar{f}\left(\right.$ resp. $\left.\bar{f}^{\prime}\right)$, and $d=F \cdot F^{\prime}$. Then $d \geq 2$; and $2 g(F)-2 \geq 2 d \cdot\left(q_{\bar{f}}-1\right)$, where $g(F)$ is the geometric genus of F. In particular,

$$
q_{\bar{f}} \leq \frac{g-1}{d}+1
$$

Proof. We first prove $d \geq 2$. If $d=1$, then it follows that $\left(\bar{f}, \bar{f}^{\prime}\right): \bar{S} \rightarrow \bar{B} \times \bar{B}^{\prime}$ is birational, which is a contradiction to the non-isotriviality of $\bar{f}$.

Now consider the restriction map

$$
\left.\bar{f}^{\prime}\right|_{F}: F \longrightarrow \bar{B}^{\prime}
$$

which is a finite morphism of degree $d$. Since $q_{\bar{f}}=g\left(B^{\prime}\right)$, according to Hurwitz formula, we get (6-6).

Remark 6.6. Xiao ([72]) has proved that if $q_{\bar{f}}=(g-1) / d+1$, then $\bar{f}$ is isotrivial.

Proof of Proposition 6.1. In order to prove (6-2), we may limit ourselves to the family $\bar{f}: \bar{S} \rightarrow \bar{B}$ coming from an admissible double cover (cf. 9] or 23]): possibly contracting some $(-2)$-curves in fibres, the family $\bar{f}$ is a double cover of a family $\bar{h}: \bar{Y} \rightarrow \bar{B}$ of stable $(2 g+2)$-pointed noded curves of arithmetic genus zero, branched along the $2 g+2$ disjoint sections $\sigma_{i}$ of $\bar{h}$ and possibly at some of the nodes of fibres of $\bar{h}$. Actually, we can get a family of admissible covers from a given $\bar{f}$ by base change unbranched over $\bar{B} \backslash \Delta$ and blow-ups of singular points in the 
fibres. These operations have the effect of multiplying all the invariants $\delta_{i}(\Upsilon)$ 's and $\xi_{j}(\Upsilon)$ 's by the same constant, and the relative irregularity $q_{\bar{f}}$ is non-decreasing under these operation.

Same as in Figure 6.2-1, let $\tilde{\pi}: \widetilde{S} \rightarrow \widetilde{Y}$ be the resolution of $\pi$ with branched divisor $\widetilde{R}$. By pullback, the disjoint sections $\sigma_{i}$ 's of $\bar{h}$ become disjoint sections of $\tilde{h}$, still denoted by $\sigma_{i}$ 's. And $\widetilde{R}$ is a union of $\widetilde{R}_{n v}:=\sum_{i=1}^{2 g+2} \sigma_{i}$ and some disjoint $(-2)$-curves contained in fibres of $\tilde{h}$.

Let $\mathfrak{S}=\left\{p_{i} \in \bar{Y}\right\}$ be the set of nodes of fibres of $\bar{h}$, and $\alpha_{i}$ (resp. $m_{i}$ ) the index (resp. multiplicity) of $p_{i} \in \mathfrak{S}$ (cf. Section 22). Let $\widetilde{\mathfrak{S}}=\left\{q_{l} \in \widetilde{Y}\right\}$ be the set of nodes of fibres of $\tilde{h}$. Define the index $\beta_{l}$ of $q_{l} \in \widetilde{\mathfrak{S}}$ to be the index of its image in $\mathfrak{S}$. Note that a node $p_{i} \in \mathfrak{S}$ of index $\alpha_{i}$ with multiplicity $m_{i}$ would introduce $m_{i}$ nodes in $\widetilde{\mathfrak{S}}$ with the same indices $\alpha_{i}$.

Let $\tilde{h}^{\prime}: \widetilde{Y} \rightarrow \bar{D}^{\prime} \cong \mathbb{P}^{1}$ be the morphism given in Proposition 6.4. Let $\tilde{\rho}: \tilde{Y} \rightarrow \hat{Y}$ be the largest contraction of 'vertical' $(-1)$-curves such that we still have an induced morphism $\hat{h}^{\prime}: \hat{Y} \rightarrow \bar{D}^{\prime}$, where 'vertical' means such a curve is mapped to a point on $\bar{B}$.

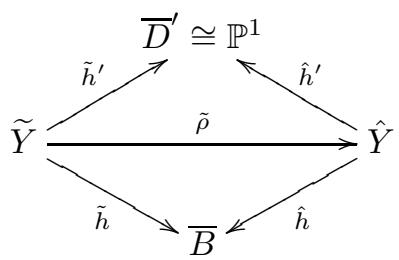

Claim 6.7. Let $\hat{R}=\tilde{\rho}(\widetilde{R})$ and $\hat{R}_{n v}=\tilde{\rho}\left(\widetilde{R}_{n v}\right)$. Then

$$
E \cdot \hat{R} \geq 2 q_{\bar{f}}+2, \quad E \cdot \hat{R}_{n v} \geq 2 q_{\bar{f}}+1, \quad \text { for any 'vertical' }(-1) \text {-curve } E \subseteq \hat{Y} \text {. }
$$

Proof of the claim. By the construction, any 'vertical' (-1)-curve $E \subseteq \hat{Y}$ is mapped surjectively onto $\bar{D}^{\prime}$ by $\hat{h}^{\prime}$. Since $\widetilde{R}$ is contained in fibres of $\tilde{h}^{\prime}$ by Proposition 6.4 $\hat{R}$ is contained in fibres of $\hat{h}^{\prime}$. Hence $E \not \subset \hat{R}$.

Note that $\hat{R}$ is the union of $\hat{R}_{n v}$ and some curves in fibres of $\hat{h}$. Let $\hat{\Gamma} \subseteq \hat{Y}$ be the fibre of $\hat{h}$ containing $E$. Then

$$
E \cdot\left(\hat{R}-\hat{R}_{n v}\right) \leq E \cdot(\hat{\Gamma}-E)=-E^{2}=1 .
$$

Therefore it suffices to prove $E \cdot \hat{R} \geq 2 q_{\bar{f}}+2$.

Let $\widetilde{E}^{\prime} \subseteq \widetilde{S}$ and $\widetilde{E} \subseteq \widetilde{Y}$ be the strict inverse image of $E$ in $\widetilde{S}$ and $\widetilde{Y}$ respectively. Then by construction, $\widetilde{E}^{\prime}$ (resp. $\widetilde{E}$ ) is mapped surjectively onto $\bar{B}^{\prime}$ (resp. $\bar{D}^{\prime}$ ) by $\tilde{f}^{\prime}$ (resp. $\tilde{h}^{\prime}$ ), and $E \cdot \hat{R} \geq \widetilde{E} \cdot \widetilde{R}$. Applying Hurwitz formula to the double cover $\widetilde{E}^{\prime} \rightarrow \widetilde{E} \cong \mathbb{P}^{1}$, whose branched locus is at most $\widetilde{E} \cap \widetilde{R}$, one gets

$$
2 g\left(\widetilde{E}^{\prime}\right)-2 \leq-4+|\widetilde{E} \cap \widetilde{R}| \text {. }
$$

As $\widetilde{E}^{\prime}$ is mapped surjectively onto $\bar{B}^{\prime}, g\left(\widetilde{E}^{\prime}\right) \geq g\left(\bar{B}^{\prime}\right)=q_{\bar{f}}$. Hence

$$
E \cdot \hat{R} \geq \widetilde{E} \cdot \widetilde{R} \geq|\widetilde{E} \cap \widetilde{R}| \geq 2 g\left(\widetilde{E}^{\prime}\right)+2 \geq 2 q_{\bar{f}}+2 .
$$

The proof is complete.

Now we contract $\hat{\rho}: \hat{Y} \rightarrow \bar{Y}$ to a $\mathbb{P}^{1}$-bundle $\bar{h}: \bar{Y} \rightarrow \bar{B}$ such that the order of every singularity of $\bar{R}_{n v}=\hat{\rho}\left(\hat{R}_{n v}\right)$ is at most $g+1$. It is easy to see that such a contraction exists. 


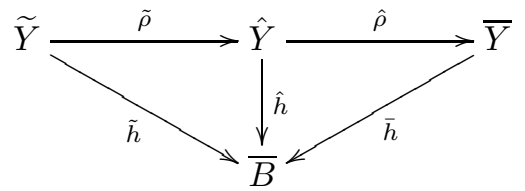

Let $\rho=\hat{\rho} \circ \tilde{\rho}$. Then $\rho$ can be viewed as a sequence of blow-ups $\rho_{l}: Y_{l} \rightarrow Y_{l-1}$ centered at $y_{l-1} \in Y_{l-1}$ with $Y_{t+s}=\widetilde{Y}, Y_{s}=\hat{Y}$, and $Y_{0}=\bar{Y}$. Let $R_{n v, l} \subseteq Y_{l}$ be the image of $\widetilde{R}_{n v}$ and $y_{l-1}$ be a singularity of $R_{n v, l-1}$ of order $n_{l-1}$. Then one sees that each blow-up $\rho_{l}$ creates a node $q \in \widetilde{\mathfrak{S}}$ with index $\beta=n_{l-1}$. Hence

$$
\widetilde{R}_{n v}^{2}=\bar{R}_{n v}^{2}-\sum_{q_{l} \in \widetilde{\mathfrak{S}}} \beta_{l}^{2} .
$$

By Claim 6.7, for $1 \leq l \leq s$, every blow-up $\rho_{l}: Y_{l} \rightarrow Y_{l-1}$ is centered at a point $y_{l-1}$ with $n_{l-1} \geq 2 q_{\bar{f}}+1$. In other words, for $1 \leq l \leq s$, each $\rho_{l}$ creates a node $q \in \widetilde{\mathfrak{S}}$ with index at least $2 q_{\bar{f}}+1$. The set $\widetilde{\mathfrak{S}}$ can be divided into two subsets $\widetilde{\mathfrak{S}}_{\hat{\rho}}$ and $\widetilde{\mathfrak{S}}_{\tilde{\rho}}$, where the first is created by blow-ups contained in $\hat{\rho}$ and the second by blow-ups contained in $\tilde{\rho}$. Then

$$
\begin{aligned}
\beta_{l} & \geq 2 q_{\bar{f}}+1, \quad \forall q_{l} \in \widetilde{\mathfrak{S}}_{\hat{\rho}} ; \\
\hat{R}_{n v}^{2} & =\bar{R}_{n v}^{2}-\sum_{q_{l} \in \widetilde{\mathfrak{S}}_{\hat{\rho}}} \beta_{l}^{2} .
\end{aligned}
$$

Note that $\widetilde{R}_{n v}$ consists of $2 g+2$ disjoint sections $\sigma_{i}$ 's. According to [9, Lemma 4.8],

$$
\widetilde{R}_{n v}^{2}=\sum_{i=1}^{2 g+2} \sigma_{i} \cdot \sigma_{i}=-\sum_{p_{i} \in \mathfrak{S}} \frac{m_{i} \alpha_{i}\left(2 g+2-\alpha_{i}\right)}{2 g+1}=-\sum_{q_{l} \in \widetilde{\mathfrak{S}}} \frac{\beta_{l}\left(2 g+2-\beta_{l}\right)}{2 g+1} .
$$

Combining (6-7), (6-9) and (6-10), one gets

$$
\hat{R}_{n v}^{2}=\widetilde{R}_{n v}^{2}+\sum_{q_{l} \in \widetilde{\mathfrak{S}}_{\tilde{\rho}}} \beta_{l}^{2}=\sum_{q_{l} \in \widetilde{\mathfrak{S}}_{\tilde{\rho}}} \frac{(2 g+2) \beta_{l}\left(\beta_{l}-1\right)}{2 g+1}-\sum_{q_{l} \in \widetilde{\mathfrak{S}}_{\hat{\rho}}} \frac{\beta_{l}\left(2 g+2-\beta_{l}\right)}{2 g+1} .
$$

Now according to Proposition [6.4, $\widetilde{R}_{n v} \subseteq \widetilde{R}$ is contained in the fibres of $\tilde{h}^{\prime}$. So $\hat{R}_{n v}=\tilde{\rho}\left(\widetilde{R}_{n v}\right)$ is contained in the fibres of $\hat{h}^{\prime}$. In particular, $\hat{R}_{n v}^{2} \leq 0$. Hence by (6-11), we obtain

$$
\sum_{q_{l} \in \widetilde{\mathfrak{S}}_{\tilde{\rho}}} \beta_{l}\left(\beta_{l}-1\right) \leq \sum_{q_{l} \in \widetilde{\mathfrak{S}}_{\hat{\rho}}} \frac{\beta_{l}\left(2 g+2-\beta_{l}\right)}{2 g+2} .
$$

Let $\epsilon_{k}$ (resp. $\nu_{k}$ ) be the number of points in $\widetilde{\mathfrak{S}}$ of index $2 k+1$ (resp. $2 k+2$ ), which is also the number of points in $\mathfrak{S}$ of index $2 k+1$ (resp. $2 k+2)$, accounted with multiplicity. Hence (cf. [9, (4.10)]),

$$
\xi_{0}(\Upsilon)=2 \nu_{0} ; \quad \delta_{i}(\Upsilon)=\epsilon_{i} / 2, \forall 1 \leq i \leq[g / 2] ; \quad \xi_{j}(\Upsilon)=\nu_{j}, \quad \forall 1 \leq j \leq[(g-1) / 2] .
$$


Therefore,

$$
\begin{aligned}
& \sum_{i=q_{\bar{f}}}^{[g / 2]} \frac{(2 i+1)(2 g+1-2 i)}{g+1} \delta_{i}(\Upsilon)+\sum_{j=q_{\bar{f}}}^{[(g-1) / 2]} \frac{2(j+1)(g-j)}{g+1} \xi_{j}(\Upsilon) \\
& =\sum_{i=q_{\bar{f}}}^{[g / 2]} \frac{(2 i+1)((2 g+2)-(2 i+1))}{2 g+2} \epsilon_{i}+\sum_{j=q_{\bar{f}}}^{[(g-1) / 2]} \frac{(2 j+2)((2 g+2)-(2 j+2))}{2 g+2} \nu_{j} \\
& =\sum \frac{\beta_{l}\left(2 g+2-\beta_{l}\right)}{2 g+2}, \quad \text { the sum is taken over all } q_{l} \in \widetilde{\mathfrak{S}} \text { with index } \beta_{l} \geq 2 q_{\bar{f}}+1 \text {, } \\
& \geq \sum_{q_{l} \in \widetilde{\mathfrak{S}}_{\hat{\rho}}} \frac{\beta_{l}\left(2 g+2-\beta_{l}\right)}{2 g+2}, \quad \text { since any point } q_{l} \in \widetilde{\mathfrak{S}}_{\hat{\rho}} \text { is of index } \beta_{l} \geq 2 q_{\bar{f}}+1 \text { by (6-8) } \\
& \geq \sum_{q_{l} \in \widetilde{\mathfrak{S}}_{\tilde{\rho}}} \beta_{l}\left(\beta_{l}-1\right), \quad \text { by }(\underline{6-12}), \\
& \begin{array}{ll}
\geq \sum \beta_{l}\left(\beta_{l}-1\right), \quad & \text { the sum is taken over all } q_{l} \in \widetilde{\mathfrak{S}} \text { with index } \beta_{l}<2 q_{\bar{f}}+1, \\
\text { and such points are all contained in } \widetilde{\mathfrak{S}}_{\tilde{\rho}} \text { by }(6-8),
\end{array} \\
& =2 \nu_{0}+\sum_{i=1}^{q_{\bar{f}}-1} 2 i(2 i+1) \epsilon_{i}+\sum_{j=1}^{q_{\bar{f}}-1} 2(j+1)(2 j+1) \nu_{j} \\
& =\xi_{0}(\Upsilon)+\sum_{i=1}^{q_{\bar{f}}-1} 4 i(2 i+1) \delta_{i}(\Upsilon)+\sum_{j=1}^{q_{\bar{f}}-1} 2(j+1)(2 j+1) \xi_{j}(\Upsilon)
\end{aligned}
$$

This completes the proof.

6.2.2. Proof of Theorem 4.3. First we consider the case that $q_{\bar{f}}=0$. By (2-5) and (2-9),

$$
\delta_{\bar{f}}=\xi_{0}(\Upsilon)+\sum_{i=1}^{[g / 2]} \delta_{i}(\Upsilon)+2 \sum_{j=1}^{[(g-1) / 2]} \xi_{j}(\Upsilon)
$$

From the above equation together with (2-2) and (6-1), it follows that

$$
\begin{aligned}
\omega \frac{2}{S} / \bar{B}= & \frac{g-1}{2 g+1} \xi_{0}(\Upsilon)+ \\
& \sum_{i=1}^{[g / 2]}\left(\frac{12 i(g-i)}{2 g+1}-1\right) \delta_{i}(\Upsilon)+\sum_{j=1}^{[(g-1) / 2]}\left(\frac{6(j+1)(g-j)}{2 g+1}-2\right) \xi_{j}(\Upsilon) .
\end{aligned}
$$

Hence, (4-3) in the case $q_{\bar{f}}=0$ is obtained as below:

$$
\begin{aligned}
& \omega_{\bar{S} / \bar{B}}^{2}-\frac{4(g-1)}{g} \cdot \operatorname{deg} \bar{f}_{*} \omega_{\bar{S} / \bar{B}} \\
& =\sum_{i=1}^{[g / 2]} \frac{4 i(g-i)-g}{g} \delta_{i}(\Upsilon)+\sum_{j=1}^{[(g-1) / 2]} \frac{2(j+1)(g-j)-2 g}{g} \xi_{j}(\Upsilon) \\
& \geq \begin{cases}\frac{3 g-4}{g} \delta_{1}(\Upsilon)+\frac{7 g-16}{g} \delta_{h}(\Upsilon), & \text { if } \Delta_{n c} \neq \emptyset ; \\
\sum_{i=1}^{[g / 2]} \frac{4 i(g-i)-g}{g} \delta_{i}(\Upsilon), & \text { if } \Delta_{n c}=\emptyset .\end{cases}
\end{aligned}
$$


Next we consider the case $q_{\bar{f}}>0$. It is based on (6-1) and (6-2). Assume that $\Delta_{n c} \neq \emptyset$. By (6-1) and (6-15), one gets

$$
\begin{aligned}
\omega_{\bar{S} / \bar{B}}^{2}-\frac{4(g-1)}{g-q_{\bar{f}}} \operatorname{deg} \bar{f}_{*} \omega_{\bar{S} / \bar{B}}= & -\frac{(g-1) q_{\bar{f}}}{(2 g+1)\left(g-q_{\bar{f}}\right)} \xi_{0}(\Upsilon) \\
& +\sum_{i=1}^{[g / 2]}\left(\frac{4\left(2 g-3 q_{\bar{f}}+1\right) i(g-i)}{(2 g+1)\left(g-q_{\bar{f}}\right)}-1\right) \delta_{i}(\Upsilon) \\
& +\sum_{j=1}^{[(g-1) / 2]}\left(\frac{2\left(2 g-3 q_{\bar{f}}+1\right)(j+1)(g-j)}{(2 g+1)\left(g-q_{\bar{f}}\right)}-2\right) \xi_{j}(\Upsilon) .
\end{aligned}
$$

Combining this with (6-2), one gets

$$
\begin{aligned}
& \omega \frac{2}{S} / \bar{B}-\frac{4(g-1)}{g-q_{\bar{f}}} \operatorname{deg} \bar{f}_{*} \omega_{\bar{S} / \bar{B}} \\
\geq & \sum_{i=1}^{q_{\bar{f}}-1} a_{i} \delta_{i}(\Upsilon)+\sum_{i=q_{\bar{f}}}^{[g / 2]} b_{i} \delta_{i}(\Upsilon)+\sum_{j=1}^{q_{\bar{f}}-1} c_{j} \xi_{j}(\Upsilon)+\sum_{j=q_{\bar{f}}}^{[(g-1) / 2]} d_{j} \xi_{j}(\Upsilon),
\end{aligned}
$$

where

$$
\left\{\begin{array}{l}
a_{i}=\left(\frac{4\left(2 g-3 q_{\bar{f}}+1\right) i(g-i)}{(2 g+1)\left(g-q_{\bar{f}}\right)}-1\right)+\frac{(g-1) q_{\bar{f}}}{(2 g+1)\left(g-q_{\bar{f}}\right)} \cdot 4 i(2 i+1), \\
b_{i}=\left(\frac{4\left(2 g-3 q_{\bar{f}}+1\right) i(g-i)}{(2 g+1)\left(g-q_{\bar{f}}\right)}-1\right)-\frac{(g-1) q_{\bar{f}}}{(2 g+1)\left(g-q_{\bar{f}}\right)} \cdot \frac{(2 i+1)(2 g+1-2 i)}{g+1}, \\
c_{j}=\left(\frac{2\left(2 g-3 q_{\bar{f}}+1\right)(j+1)(g-j)}{(2 g+1)\left(g-q_{\bar{f}}\right)}-2\right)+\frac{(g-1) q_{\bar{f}}}{(2 g+1)\left(g-q_{\bar{f}}\right)} \cdot 2(j+1)(2 j+1), \\
d_{j}=\left(\frac{2\left(2 g-3 q_{\bar{f}}+1\right)(j+1)(g-j)}{(2 g+1)\left(g-q_{\bar{f}}\right)}-2\right)-\frac{(g-1) q_{\bar{f}}}{(2 g+1)\left(g-q_{\bar{f}}\right)} \cdot \frac{2(j+1)(g-j)}{g+1} .
\end{array}\right.
$$

If $q_{\bar{f}}=1$, then

$$
\begin{aligned}
b_{1} & =\frac{3 g-6}{g+1} ; \\
b_{i} & =\frac{4 i(g-i)-g-2}{g+1} \geq \frac{7 g-18}{g+1}, \\
d_{j} & =\frac{2((j+1)(g-j)-(g+1))}{g+1} \geq 0, \quad \forall 2 \leq i \leq[g / 2] ;
\end{aligned}
$$

If $q_{\bar{f}} \geq 2$, then

$$
\begin{array}{ll}
a_{1} \geq \frac{3 g^{2}-\left(8 q_{\bar{f}}+1\right) g+10 q_{\bar{f}}-4}{(g+1)\left(g-q_{\bar{f}}\right)} ; & \\
a_{i} \geq \frac{7 g^{2}-\left(16 q_{\bar{f}}+9\right) g+34 q_{\bar{f}}-16}{(g+1)\left(g-q_{\bar{f}}\right)}, & \forall 2 \leq i \leq q_{\bar{f}}-1 ; \\
b_{i} \geq \frac{7 g^{2}-\left(16 q_{\bar{f}}+9\right) g+34 q_{\bar{f}}-16}{(g+1)\left(g-q_{\bar{f}}\right)}, & \forall q_{\bar{f}} \leq i \leq[g / 2] ; \\
c_{j} \geq 0, & \forall 1 \leq j \leq q_{\bar{f}}-1 ; \\
d_{j} \geq 0, & \forall q_{\bar{f}} \leq j \leq[(g-1) / 2] .
\end{array}
$$

Hence (4-3) holds for $\Delta_{n c} \neq \emptyset$. 
Now we consider the case that $\Delta_{n c}=\emptyset$. Note that in this case,

$$
\xi_{j}(\Upsilon)=0, \quad \forall 0 \leq j \leq[(g-1) / 2] .
$$

Hence by (6-1) and (6-15), we get

$$
\omega_{\bar{S} / \bar{B}}^{2}-\frac{4(g-1)}{g-q_{\bar{f}}} \operatorname{deg} \bar{f}_{*} \omega_{\bar{S} / \bar{B}}=\sum_{i=1}^{[g / 2]}\left(\frac{4\left(2 g+1-3 q_{\bar{f}}\right) i(g-i)}{(2 g+1)\left(g-q_{\bar{f}}\right)}-1\right) \delta_{i}(\Upsilon) .
$$

Hence (4-3) holds. If moreover $q_{\bar{f}} \geq 2$, then according to (6-2) and (6-16), we get

$$
\sum_{i=q_{\bar{f}}}^{[g / 2]} \frac{(2 i+1)(2 g+1-2 i)}{g+1} \cdot \delta_{i}(\Upsilon) \geq \sum_{i=1}^{q_{\bar{f}}-1} 4 i(2 i+1) \cdot \delta_{i}(\Upsilon)
$$

So (4-4) is proved.

6.3. Proof of Theorem 4.5. It is based on analyzing the following natural multiplication

$$
\varrho: S^{2}\left(\bar{f}_{*} \omega_{\bar{S} / \bar{B}}\right) \longrightarrow \bar{f}_{*}(\omega \bar{S} / \bar{B})
$$

where $S^{2}\left(\bar{f}_{*} \omega_{\bar{S} / \bar{B}}\right)$ is the symmetric power of $\bar{f}_{*} \omega_{\bar{S}} / \bar{B}$.

Proof of Theorem 4.5. As $\bar{f}$ is non-hyperelliptic, the morphism $\varrho$ in (6-17) is generically surjective. One gets an exact sequence as below:

$$
0 \longrightarrow \mathcal{R} \longrightarrow S^{2}\left(\bar{f}_{*} \omega_{\bar{S}} / \bar{B}\right) \stackrel{\varrho}{\longrightarrow} \bar{f}_{*}\left(\omega_{\bar{S} / \bar{B}}^{\otimes 2}\right) \mathcal{S} \longrightarrow 0,
$$

where $\mathcal{R}$ and $\mathcal{S}$ are the kernel and cokernel of $\varrho$, and $\mathcal{S}$ is a torsion module. So

$$
\operatorname{deg} \mathcal{R}+\operatorname{deg} \bar{f}_{*}\left(\omega_{\bar{S} / \bar{B}}^{\otimes 2}\right)=\operatorname{deg} S^{2}\left(\bar{f}_{*} \omega_{\bar{S} / \bar{B}}\right)+\operatorname{deg} \mathcal{S} .
$$

It is not difficult to show that

$$
\begin{aligned}
\operatorname{deg} S^{2}\left(\bar{f}_{*} \omega_{\bar{S} / \bar{B}}\right) & =(g+1) \operatorname{deg} \bar{f}_{*} \omega_{\bar{S} / \bar{B}}, \\
\operatorname{deg} \bar{f}_{*}\left(\omega_{\bar{S} / \bar{B}}^{\otimes 2}\right) & =\omega_{\bar{S} / \bar{B}}^{2}+\operatorname{deg} \bar{f}_{*} \omega_{\bar{S} / \bar{B}} .
\end{aligned}
$$

Since $\bar{f}_{*} \omega_{\bar{S} / \bar{B}}$ is semi-stable, so is $S^{2}\left(\bar{f}_{*} \omega_{\bar{S} / \bar{B}}\right)$ of slope $\mu_{2}=\frac{2 \operatorname{deg} \bar{f}_{*} \omega_{\bar{S} / \bar{B}}}{g}$. Note that

$$
\operatorname{rank} \mathcal{R}=\operatorname{rank} S^{2}\left(\bar{f}_{*} \omega_{\bar{S} / \bar{B}}\right)-\operatorname{rank} \bar{f}_{*}\left(\omega_{\bar{S} / \bar{B}}^{\otimes 2}\right)=\frac{(g-2)(g-3)}{2} .
$$

Hence for the subsheaf $\mathcal{R} \subseteq S^{2}\left(\bar{f}_{*} \omega_{\bar{S} / \bar{B}}\right)$, we have

$$
\operatorname{deg} \mathcal{R} \leq \operatorname{rank} \mathcal{R} \cdot \mu_{2}=\frac{(g-2)(g-3)}{g} \cdot \operatorname{deg} \bar{f}_{*} \omega_{\bar{S} / \bar{B}}
$$

Since $\mathcal{S}$ is a torsion module,

$$
\operatorname{deg} \mathcal{S}=\sum_{p} \text { length } \mathcal{S}_{p}
$$

Therefore, by (6-18), (6-19), (6-20), (6-21) and (6-22), it suffices to prove that

$$
\text { length } \mathcal{S}_{p} \geq \begin{cases}3\left(l_{h}\left(F_{p}\right)-1\right)+2 l_{1}\left(F_{p}\right), & \forall p \in \Delta_{c t} \backslash \Lambda, \\ 2(g-2)+2\left(l_{h}\left(F_{p}\right)+l_{1}\left(F_{p}\right)-1\right), & \forall p \in \Lambda,\end{cases}
$$

which follows from Lemmas 6.8 and 6.10. Indeed, if $p \in \Delta_{c t} \backslash \Lambda$, then (6-23) follows from (6-25) and (6-28); if $p \in \Lambda$, then $F_{p}$ is hyperelliptic, and so (6-23) follows from (6-26) and (6-29). This completes the proof. 
Lemma 6.8. For every $p \in \bar{B} \backslash \Delta_{n c}$, let

$$
\nu_{p}: S^{2} H^{0}\left(F_{p}, \omega_{F_{p}}\right) \rightarrow H^{0}\left(F_{p}, \omega_{F_{p}}^{\otimes 2}\right)
$$

the natural multiplication map on $F_{p}$, and denote by coker $\left(\nu_{p}\right)$ the cokernel of $\nu_{p}$. Then

$$
\operatorname{dim} \operatorname{coker}\left(\nu_{p}\right) \geq 3\left(l_{h}\left(F_{p}\right)-1\right)+2 l_{1}\left(F_{p}\right) .
$$

If $F_{p}$ is hyperelliptic, then

$$
\operatorname{dim} \operatorname{coker}\left(\nu_{p}\right) \geq(g-2)+\left(l_{h}\left(F_{p}\right)+l_{1}\left(F_{p}\right)-1\right) .
$$

Proof. Assume $F_{p}=\sum_{i} C_{i}$. Let $\operatorname{pr}_{i}: F_{p} \rightarrow C_{i}$ be the natural contraction map to $C_{i}$. By pulling-back, one may view $H^{0}\left(C_{i}, \omega_{C_{i}}\right)$ as a subspace of $H^{0}\left(F_{p}, \omega_{F_{p}}\right)$, and

$$
H^{0}\left(F_{p}, \omega_{F_{p}}\right) \cong \bigoplus_{i} H^{0}\left(C_{i}, \omega_{C_{i}}\right)
$$

Note that for $\omega \in H^{0}\left(F_{p}, \omega_{F_{p}}\right)$ which lies in $H^{0}\left(C_{i}, \omega_{C_{i}}\right)$ in the above decomposition, we have $\left.\omega\right|_{C_{j}}=0$ for all $j \neq i$. Thus the map $\nu_{p}$ factors through

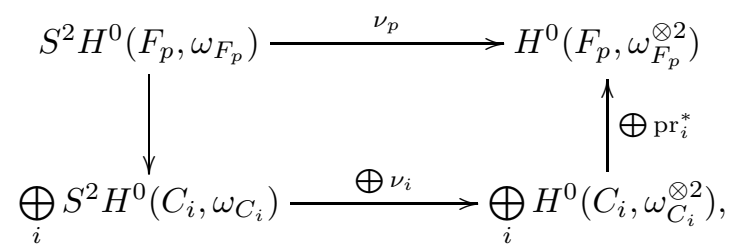

where $\nu_{i}: S^{2} H^{0}\left(C_{i}, \omega_{C_{i}}\right) \rightarrow H^{0}\left(C_{i}, \omega_{C_{i}}^{\otimes 2}\right)$ is the natural multiplication map. Note that for a smooth closed curve $C_{i}$ of genus $g\left(C_{i}\right)$,

$$
\operatorname{dim} \nu_{i}\left(S^{2} H^{0}\left(C_{i}, \omega_{C_{i}}\right)\right) \leq \begin{cases}3 g\left(C_{i}\right)-3, & \text { if } g\left(C_{i}\right) \geq 2 ; \\ 3 g\left(C_{i}\right)-2=1, & \text { if } g\left(C_{i}\right)=1 ; \\ 3 g\left(C_{i}\right)=0, & \text { if } g\left(C_{i}\right)=0 .\end{cases}
$$

Since $p \in \bar{B} \backslash \Delta_{n c}, F_{p}$ has compact Jacobian, then $\sum_{i} g\left(C_{i}\right)=g$. Hence

$\operatorname{dim} \nu_{p}\left(S^{2} H^{0}\left(F_{p}, \omega_{F_{p}}\right)\right) \leq \sum_{i} 3 g\left(C_{i}\right)-3 l_{h}\left(F_{p}\right)-2 l_{1}\left(F_{p}\right)=3 g-3 l_{h}\left(F_{p}\right)-2 l_{1}\left(F_{p}\right)$.

Since $\operatorname{dim} H^{0}\left(F_{p}, \omega_{F_{p}}^{\otimes 2}\right)=3 g-3$, fo-25 follows immediately.

If $F_{p}$ is hyperelliptic, then each component $C_{i} \subseteq F_{p}$ with $g\left(C_{i}\right) \geq 2$ must be hyperelliptic. Thus it follows that

$$
\operatorname{dim} \nu_{i}\left(S^{2} H^{0}\left(C_{i}, \omega_{C_{i}}\right)\right)= \begin{cases}2 g\left(C_{i}\right)-1, & \text { if } g\left(C_{i}\right) \geq 1 \\ 2 g\left(C_{i}\right)=0, & \text { if } g\left(C_{i}\right)=0\end{cases}
$$

So

$$
\operatorname{dim} \nu_{p}\left(S^{2} H^{0}\left(F_{p}, \omega_{F_{p}}\right)\right)=\sum_{i} 2 g\left(C_{i}\right)-l_{h}\left(F_{p}\right)-l_{1}\left(F_{p}\right)=2 g-l_{h}\left(F_{p}\right)-l_{1}\left(F_{p}\right) .
$$

Hence $\underline{6-26)}$ follows.

Lemma 6.9. For $p \in \Lambda$, there exists a neighborhood $p \in W \subseteq \bar{B}$ with local coordinate $t$ and local sections $s_{1}, \cdots, s_{g}$ of $\bar{f}_{*} \omega_{\bar{S} / \bar{B}}$ such that every $s_{i}$ is a function of $t^{2}$ and $H^{0}\left(F_{p}, \omega_{F_{p}}\right)$ is generated by $\left\{\varphi\left(s_{1}\right), \cdots, \varphi\left(s_{g}\right)\right\}$, where $\varphi$ is defined as below:

$$
\varphi: H^{0}\left(W, \bar{f}_{*} \omega_{\bar{S} / \bar{B}}\right) \stackrel{\varphi_{1}}{\longrightarrow} H^{0}\left(\bar{f}^{-1}(W), \omega_{\bar{S} / \bar{B}}\right) \stackrel{\varphi_{2}}{\longrightarrow} H^{0}\left(F_{q}, \omega_{F_{q}}\right) .
$$


Proof. Recall the logarithmic Higgs bundle on $\bar{B}$ (resp. $\bar{C}$ )

$$
\left(E_{\bar{B}}^{1,0} \oplus E_{\bar{B}}^{0,1}, \theta_{\bar{B}}\right) \quad \operatorname{resp} .\left(E_{\bar{C}}^{1,0} \oplus E_{\bar{C}}^{0,1}, \theta_{\bar{C}}\right) .
$$

By (3-4),

$$
E_{\bar{B}}^{1,0}=\bar{f}_{*} \omega_{\bar{S} / \bar{B}}=\bar{j}_{B}^{*} E_{\bar{C}}^{1,0}
$$

Moreover, as $\Lambda$ is the ramification locus of $j_{B}=\left.\left(\bar{j}_{B}\right)\right|_{B}: B \rightarrow C$, it follows that around each $p \in \Lambda, \bar{f}_{*} \omega_{\bar{S}} / \bar{B}$ can be locally generated by sections which are functions of $t^{2}$, where $t$ is a suitable local coordinate of $\bar{B}$. The map $\varphi$ is obviously surjective.

Lemma 6.10. For every $p \in \bar{B} \backslash \Delta_{n c}$, we have

$$
\text { length } \mathcal{S}_{p} \geq \operatorname{dim} \operatorname{coker}\left(\nu_{p}\right) \text {. }
$$

If $p \in \Lambda$, then

$$
\text { length } \mathcal{S}_{p} \geq 2 \operatorname{dim} \operatorname{coker}\left(\nu_{p}\right) \text {. }
$$

Proof. Let $\mathfrak{m}_{p}$ be the ideal of $p \in \bar{B}$. Then we have the following natural isomorphism

$$
\varrho\left(S^{2}\left(\bar{f}_{*} \omega_{\bar{S}} / \bar{B}\right)\right) /\left(\mathfrak{m}_{p} \cdot \varrho\left(S^{2}\left(\bar{f}_{*} \omega_{\bar{S}} / \bar{B}\right)\right)\right) \cong \nu_{p}\left(S^{2} H^{0}\left(F_{p}, \omega_{F_{p}}\right)\right)
$$

It follows that $\mathcal{S}_{p} / \mathfrak{m}_{p} \cdot \mathcal{S}_{p} \cong \operatorname{coker}\left(\nu_{p}\right)$. Hence

$$
\operatorname{length} \mathcal{S}_{p} \geq \operatorname{dim}\left(\mathcal{S}_{p} / \mathfrak{m}_{p} \cdot \mathcal{S}_{p}\right)=\operatorname{dim} \operatorname{coker}\left(\nu_{p}\right)
$$

If $p \in \Lambda$, then according to Lemma 6.9 and its proof, $\bar{f}_{*} \omega_{\bar{S}} / \bar{B}$ is generated by local sections which are functions in $t^{2}$ around $p$, where $t$ is a local coordinate of $\bar{B}$ around $p$. Hence the image of $\varrho$ is also generated by local sections which are functions in $t^{2}$ around $p$. This implies in particular that

$$
\operatorname{dim}\left(\mathcal{S}_{p} / \mathfrak{m}_{p}^{2} \cdot \mathcal{S}_{p}\right)=2 \operatorname{dim}\left(\mathcal{S}_{p} / \mathfrak{m}_{p} \cdot \mathcal{S}_{p}\right) .
$$

Hence

$$
\text { length } \mathcal{S}_{p} \geq \operatorname{dim}\left(\mathcal{S}_{p} / \mathfrak{m}_{p}^{2} \cdot \mathcal{S}_{p}\right)=2 \operatorname{dim}\left(\mathcal{S}_{p} / \mathfrak{m}_{p} \cdot \mathcal{S}_{p}\right)=2 \operatorname{dim} \operatorname{coker}\left(\nu_{p}\right) .
$$

\section{Flat PART OF $R^{1} \bar{f}_{*} \mathbb{Q}$ FOR A FAMILY OF HYPERELLIPTIC SEMI-STABLE CURVES}

We are going to prove Theorem 4.7 based on Lemma 7.1 regarding the global invariant cycle with unitary locally constant coefficient and Bogomolov's lemma (cf. [62, Lemma 7.5]) concerning the Kodaira dimension of an invertible subsheaf of the sheaf of logarithmic differential forms on a smooth projective surface. Lemma 7.1 comes from a discussion with Chris Peters and is obtained by generalizing Deligne's original theorem with the constant coefficient. For technical reasons, we consider $\mathbb{C}$-local systems instead of $\mathbb{Q}$-local systems.

Lemma 7.1. Let $f: X^{0} \rightarrow \bar{B} \backslash \Delta$ be a smooth projective morphism. Let $X \supseteq X^{0}$ be a smooth compactification of $X^{0}$ and $\mathbb{U}$ be a locally constant sheaf on $X$ coming from a representation of $\pi_{1}(X)$ into a unitary group. Then the following canonical homomorphism is surjective:

$$
H^{k}(X, \mathbb{U}) \longrightarrow H^{0}\left(\bar{B} \backslash \Delta, R^{k} f_{*} \mathbb{U}\right) .
$$


Proof. We will follow Deligne's proof for the case that $\mathbb{U}=\mathbb{Q}(\operatorname{cf.}[10, \S 4.1])$ verbatim.

The unitary locally constant sheaf $\mathbb{U}$ on $X$ naturally underlies a polarized variation of Hodge structure, say, of pure type $(0,0)$. Hence it follows from M. Saito's theory of polarizable Hodge modules that there is an induced pure Hodge structure of weight $k$ on $H^{k}(X, \mathbb{U})$ as well as on $H^{k}\left(X_{b},\left.\mathbb{U}\right|_{X_{b}}\right)$ where $X_{b}$ is any (smooth projective) fibre of $f: X^{0} \rightarrow \bar{B} \backslash \Delta$.

We first show the "edge-homomorphism"

$$
p_{e}: H^{k}\left(X^{0}, \mathbb{U}\right) \longrightarrow H^{0}\left(\bar{B} \backslash \Delta, R^{k} f_{*} \mathbb{U}\right)
$$

is surjective by the following argument from the proof of [59, Proposition 1.38].

Indeed, if we take $h \in H^{2}\left(X^{0}, \mathbb{Q}\right)$ to be the restriction of a hyperplane class of $X$, then it suffices to show that the cup-products satisfy the hard Lefschetz property, i.e., the following homomorphism is an isomorphism for any $0 \leq k \leq m$, where $m$ is the dimension of a general fibre of $f$ :

$$
[\cup h]^{k}: R^{m-k} f_{*} \mathbb{U} \longrightarrow R^{m+k} f_{*} \mathbb{U} .
$$

Note that the hard Lefschetz property can be verified fiber-by-fiber. On each fiber the natural locally constant metric on $\mathbb{U}$ induces a Hodge decomposition of the cohomology with coefficients in $\mathbb{U}$, hence the hard Lefschetz property holds. So $p_{e}$ in (7-1) is surjective.

Since the restriction homomorphism (as monodromy invariant)

$$
H^{0}\left(\bar{B} \backslash \Delta, R^{k} f_{*} \mathbb{U}\right) \rightarrow H^{k}\left(X_{b},\left.\mathbb{U}\right|_{X_{b}}\right)
$$

is injective and $H^{k}\left(X_{b},\left.\mathbb{U}\right|_{X_{b}}\right)$ carries a pure Hodge structure of weight- $k$, one gets that $H^{0}\left(\bar{B} \backslash \Delta, R^{k} f_{*} \mathbb{U}\right)$ carries a pure Hodge structure of weight- $k$. The surjectivity of $p_{e}$ in (7-1) also induces surjective morphisms between the weight-filtrations of the both cohomologies. In particular, we have a surjective homomorphism

$$
W_{k}\left(H^{k}\left(X^{0}, \mathbb{U}\right)\right) \rightarrow W_{k}\left(H^{0}\left(\bar{B} \backslash \Delta, R^{k} f_{*} \mathbb{U}\right)\right)=H^{0}\left(\bar{B} \backslash \Delta, R^{k} f_{*} \mathbb{U}\right) .
$$

By $\left[58, W_{k}\left(H^{k}\left(X^{0}, \mathbb{U}\right)\right)\right.$ is nothing but the image of the restriction homomorphism

$$
H^{k}(X, \mathbb{U}) \rightarrow H^{k}\left(X^{0}, \mathbb{U}\right) .
$$

Combining this with (17-2), one gets required surjective homomorphism.

Corollary 7.2. Let $\bar{f}: \bar{S} \rightarrow \bar{B}$ be a semi-stable family of projective curves (not necessarily hyperelliptic) over a smooth projective curve $\bar{B}$, with semi-stable singular fibres $\Upsilon \rightarrow \Delta$. Given any vector subbundle $\mathcal{U} \hookrightarrow \bar{f}_{*} \omega_{\bar{S}} / \bar{B}=\bar{f}_{*} \Omega \frac{1}{S} / \bar{B}(\log \Upsilon)$, which is a flat subbundle and is induced by locally constant subsheaf $\mathbb{U} \hookrightarrow \mathbb{V}_{\bar{B} \backslash \Delta}:=$ $R^{1} \bar{f}_{*} \mathbb{C}_{\bar{f}-1(\bar{B} \backslash \Delta)}$, it lifts to a morphism

$$
\bar{f}^{*} \mathcal{U} \longrightarrow \Omega \frac{1}{S}
$$

such that the induced canonical morphism

$$
\mathcal{U} \rightarrow \bar{f}_{*} \Omega \frac{1}{S} \rightarrow \bar{f}_{*} \Omega \frac{1}{S}(\log \Upsilon) \rightarrow \bar{f}_{*} \Omega \frac{1}{S} / \bar{B}(\log \Upsilon)
$$

coincides with the inclusion $\mathcal{U} \hookrightarrow \bar{f}_{*} \Omega \frac{1}{S} / \bar{B}(\log \Upsilon)$.

Proof. Since the local monodromy of $\mathbb{V}_{\bar{B} \backslash \Delta}$ around $\Delta$ is unipotent and the local monodromy of the subsheaf $\mathbb{U}$ around $\Delta$ is semisimple, $\mathbb{U}$ extends on $\bar{B}$ as a locally constant sheaf. The inclusion $\mathbb{U} \hookrightarrow \mathbb{V}_{\bar{B} \backslash \Delta}$ corresponds to a section

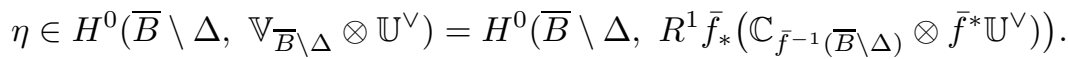


By Lemma 7.1, $\eta$ lifts to a class $\tilde{\eta} \in H^{1}\left(\bar{S}, \bar{f}^{*} \mathbb{U}^{\vee}\right)$ under the canonical morphism

$$
H^{1}\left(\bar{S}, \bar{f}^{*} \mathbb{U}^{\vee}\right) \longrightarrow H^{0}\left(\bar{B} \backslash \Delta, R^{1} \bar{f}_{*}\left(\mathbb{C}_{\bar{f}^{-1}(\bar{B} \backslash \Delta)} \otimes \bar{f}^{*} \mathbb{U}^{\vee}\right)\right) .
$$

Note that this canonical morphism is a morphism between pure Hodge structures of weight one, and by the construction $\eta$ is of type $(1,0)$, so $\tilde{\eta}$ is of type $(1,0)$, i.e.,

$$
\tilde{\eta} \in H^{0}\left(\bar{S}, \Omega \frac{1}{S} \otimes \bar{f}^{*} \mathcal{U}^{\vee}\right)
$$

which corresponds to a morphism $\bar{f}^{*} \mathcal{U} \rightarrow \Omega \frac{1}{S}$, such that under the canonical morphism (7-4) it goes back to the inclusion $\mathcal{U} \hookrightarrow \bar{f}_{*} \Omega \frac{1}{S} / \bar{B}(\log \Upsilon)$.

Let $\tilde{\pi}: \widetilde{S} \rightarrow \tilde{Y}$ be the smooth double cover described in Figure 6.2-1 and $\vartheta: \widetilde{S} \rightarrow \bar{S}$ be the blow-ups. Given any vector subbundle $\mathcal{U} \hookrightarrow \bar{f}_{*} \Omega \frac{1}{S} / \bar{B}(\log \Upsilon)$ as in Corollary 7.2 , by pulling back of (7-3), we obtain a sheaf morphism

$$
\tilde{f}^{*} \mathcal{U}=\vartheta^{*} \bar{f}^{*} \mathcal{U} \longrightarrow \Omega_{\widetilde{S}}^{1}, \quad \text { where } \tilde{f}=\bar{f} \circ \vartheta,
$$

which corresponds to an element

$$
\tilde{\eta} \in H^{0}\left(\widetilde{S}, \Omega_{\widetilde{S}}^{1} \otimes \tilde{f}^{*} \mathcal{U}^{\vee}\right) .
$$

By pushing-out, we also obtain an element (where $\tilde{h}: \tilde{Y} \rightarrow \bar{B}$ is the induced morphism)

$$
\tilde{\pi}_{*}(\tilde{\eta}) \in H^{0}\left(\widetilde{Y}, \tilde{\pi}_{*}\left(\Omega_{\widetilde{S}}^{1} \otimes \tilde{f}^{*} \mathcal{U}^{\vee}\right)\right)=H^{0}\left(\tilde{Y}, \tilde{\pi}_{*} \Omega_{\widetilde{S}}^{1} \otimes \tilde{h}^{*} \mathcal{U}^{\vee}\right)
$$

So one gets a morphism of sheaves $\tilde{h}^{*} \mathcal{U} \longrightarrow \tilde{\pi}_{*} \Omega_{\widetilde{S}}^{1}$. The Galois group $\operatorname{Gal}(\widetilde{S} / \widetilde{Y}) \cong \mathbb{Z}_{2}$ acts on $\tilde{\pi}_{*} \Omega_{\widetilde{S}}^{1}$. Hence one obtains the eigenspace decomposition

$$
\tilde{h}^{*}(\mathcal{U}) \longrightarrow\left(\tilde{\pi}_{*} \Omega_{\widetilde{S}}^{1}\right)_{1}, \quad \tilde{h}^{*}(\mathcal{U}) \longrightarrow\left(\tilde{\pi}_{*} \Omega_{\widetilde{S}}^{1}\right)_{-1} .
$$

Lemma 7.3. The image of the map

$$
\varrho: \tilde{h}^{*}(\mathcal{U}) \longrightarrow\left(\tilde{\pi}_{*} \Omega_{\widetilde{S}}^{1}\right)_{-1}
$$

is an invertible subsheaf $M$ such that $M$ is numerically effective (nef), $M^{2}=0$, and $M \cdot D=0$ for any component $D$ of the branch divisor $\widetilde{R} \subseteq \widetilde{Y}$ of the double cover $\tilde{\pi}: \widetilde{S} \rightarrow \widetilde{Y}$.

Proof. First of all, we show that $\varrho \neq 0$. It is known that

$$
\left(\tilde{\pi}_{*} \Omega_{\widetilde{S}}^{1}\right)_{1}=\Omega_{\widetilde{Y}}^{1}, \quad\left(\tilde{\pi}_{*} \Omega_{\widetilde{S}}^{1}\right)_{-1}=\Omega_{\widetilde{Y}}^{1}(\log (\widetilde{R}))(-\widetilde{L}),
$$

where $\widetilde{R} \equiv 2 \widetilde{L}$ (三 stands for linearly equivalent) is the defining data of the double cover $\tilde{\pi}: \widetilde{S} \rightarrow \widetilde{Y}$. Note that by Corollary 7.2 , the induced map

$$
\begin{aligned}
\mathcal{U}=\tilde{h}_{*} \tilde{h}^{*} \mathcal{U} \longrightarrow & \tilde{h}_{*}\left(\left(\tilde{\pi}_{*} \Omega_{\widetilde{S}}^{1}\right)_{1} \bigoplus\left(\tilde{\pi}_{*} \Omega_{\widetilde{S}}^{1}\right)_{-1}\right) \\
= & \tilde{h}_{*}\left(\Omega_{\widetilde{Y}}^{1}(\log (\widetilde{R}))(-\widetilde{L})\right) \hookrightarrow \bar{f}_{*} \Omega \frac{1}{S / B}(\log \Upsilon)
\end{aligned}
$$

is just the inclusion $\mathcal{U} \hookrightarrow \bar{f}_{*} \Omega \frac{1}{S} / \bar{B}(\log \Upsilon)$. Hence in particular, $\varrho \neq 0$.

Second, we prove that the image of $\varrho$ is a subsheaf of rank one. Otherwise, it is of rank two, and so the second wedge product

$$
\wedge^{2} \tilde{h}^{*} \mathcal{U} \stackrel{\wedge^{2} \varrho}{\longrightarrow} \wedge^{2}\left(\tilde{\pi}_{*} \Omega_{\widetilde{S}}^{1}\right)_{-1}=\omega_{\widetilde{Y}}
$$


is a non-zero map. Note that the image of the map is a quotient sheaf of $\wedge^{2} \tilde{h}^{*} \mathcal{U}$ coming from a unitary local system, so the image sheaf is semi-positive. But this is impossible, because $\omega_{\tilde{Y}}$ can not contain any non-zero semi-positive subsheaf.

Finally, we show that the image of $\varrho$ is an invertible sheaf. Suppose on the contrary the image of $\varrho$ is of the form $M \otimes I_{Z}$, where $M$ is an invertible subsheaf and $\operatorname{dim} Z=0$ with $Z \neq \emptyset$. By a suitable blow-up $\rho: X \rightarrow \widetilde{Y}$, we may assume the image of $\rho^{*} \tilde{h}^{*} \mathcal{U}$ is $\rho^{*}(M) \otimes \mathcal{O}_{X}(-E)$, where $E>0$ is a combination of the exceptional curves. As $\mathcal{U}$ comes from a unitary local system, we get that $\rho^{*}(M) \otimes \mathcal{O}_{X}(-E)$ is semi-positive and so

$$
0 \leq\left(\rho^{*}(M)-E\right)^{2}=M^{2}+E^{2} .
$$

Hence $M$ is also semi-positive and $M^{2} \geq-E^{2}>0$, which implies that the Kodaira dimension of $M$ is two. However, by (17-5), we get the following inclusion of sheaves,

$$
\mathcal{O}_{\widetilde{Y}}(\widetilde{L}) \otimes M \hookrightarrow \Omega_{\widetilde{Y}}^{1}(\log (\widetilde{R})) .
$$

As $2 \widetilde{L} \equiv \widetilde{R}$ is effective, the Kodaira dimension of $\widetilde{L} \otimes M$ is also two, which is impossible by Bogomolov's lemma (cf. 62, Lemma 7.5]).

Hence the image of $\varrho$ is an invertible subsheaf $M$, which is semi-positive since it is a quotient sheaf of a vector bundle coming from a unitary local system. And we still have the inclusion (7-7). So again by Bogomolov's lemma, we get $M^{2}=$ 0 , and $M \cdot D=0$.

Proposition 7.4. After a suitable base change unbranched over $\bar{B} \backslash \Delta, F_{\bar{B}}^{1,0}$ is trivial, i.e.,

$$
F_{\bar{B}}^{1,0}=\bigoplus_{i=1}^{r} \mathcal{O}_{\bar{B}}, \quad \text { where } r=\operatorname{rank} F_{\bar{B}}^{1,0} .
$$

Proof. Similarly to the proof of Proposition 6.1 on Page 32, we may restrict ourselves to the situation that the double cover $\pi: \bar{S} \rightarrow \bar{Y}$ induced by the hyperelliptic involution is an admissible double cover. Then the branched divisor $\widetilde{R} \subseteq \widetilde{Y}$ of the smooth double cover $\tilde{\pi}: \widetilde{S} \rightarrow \widetilde{Y}$ in Figure 6.2-1 is a union of $2 g+2$ sections and some curves contained in fibres of $\tilde{h}: \widetilde{Y} \rightarrow \bar{B}$. Let $D$ be such a section, and

$$
F_{\bar{B}}^{1,0}=\bigoplus_{i=1}^{t} \mathcal{U}_{i}, \quad \text { with each } \mathcal{U}_{i} \text { irreducible. }
$$

We first prove $\operatorname{rank} \mathcal{U}_{i}=1$ for any $i$. Assume on the contrary that rank $\mathcal{U}_{i} \geq 2$ for some $i$. Let $f: S \rightarrow B=\bar{B} \backslash \Delta_{n c}$ be the largest part of $\bar{f}$ such that the relative Jacobian is smooth. Then

$$
\left.\left(\mathbb{V}_{B}:=R^{1} j a c(f)_{*} \mathbb{C}_{J a c(S / B)}\right)\right|_{\bar{B} \backslash \Delta} \cong \mathbb{V}_{\bar{B} \backslash \Delta}:=R^{1} f_{*} \mathbb{C}_{f^{-1}(\bar{B} \backslash \Delta)} .
$$

Hence the inclusion

$$
\mathcal{U}_{i} \hookrightarrow F_{\bar{B}}^{1,0} \hookrightarrow \bar{f}_{*} \omega_{\bar{S} / \bar{B}}=\bar{f}_{*} \Omega \frac{1}{S} / \bar{B}(\log \Upsilon)
$$

underlies a unitary locally constant subsheaf $\mathbb{U}_{i} \hookrightarrow \mathbb{V}_{\bar{B} \backslash \Delta}:=R^{1} f_{*} \mathbb{C}_{f^{-1}(\bar{B} \backslash \Delta)}$. By Lemma 7.3 with $\mathcal{U}=\mathcal{U}_{i}$, we obtain $M \cdot D=0$, i.e., $\operatorname{deg} \mathcal{O}_{D}(M)=0$. As $D$ is a section, $D \cong \bar{B}$. Hence we may view $\mathcal{F}:=\mathcal{O}_{D}(M)$ as an invertible sheaf on $\bar{B}$, which is a quotient of $\mathcal{U}$ for $M$ is a quotient of $\tilde{h}^{*} \mathcal{U}$. As $\mathcal{U}$ comes from a unitary local system, $\mathcal{U}$ is poly-stable. Thus $\mathcal{U}=\mathcal{F} \oplus \mathcal{U}^{\prime}$ contradicting the irreducibility of $\mathcal{U}$. Hence $\operatorname{rank} \mathcal{U}_{i}=1$ as required.

Now applying [10, $\S 4.2]$ or [3, Theorem 3.4], we get that $\mathcal{U}_{i}$ is torsion in $\operatorname{Pic}^{0}(\bar{B})$. So after a further suitable finite étale base change, $\mathcal{U}_{i} \cong \mathcal{O}_{\bar{B}}$ for any $i$ as required. 
Proof of Theorem 4.7. Because of (7-9), the flat part $F_{\bar{B}}^{1,0}$ in the decomposition (3-3) is a flat vector bundle underlying a unitary local subsystem of $\mathbb{V}_{\bar{B} \backslash \Delta}$ coming from a representation of the fundamental group into a unitary group of rank $r=$ $\operatorname{rank} F^{1,0}$ :

$$
\tilde{\rho}_{F}: \pi_{1}(\bar{B} \backslash \Delta) \rightarrow U(r) .
$$

Note that the monodromy around $\Delta$ is unipotent, since $\bar{f}$ is semi-stable. Hence $\tilde{\rho}_{F}$ actually factors through $\pi_{1}(\bar{B})$ :

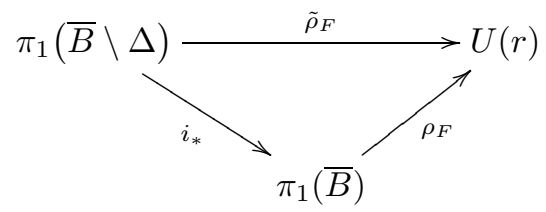

From Proposition 7.4 it follows that the image of $\tilde{\rho}_{F}$ is finite. Because $\tilde{\rho}_{F}$ factors through $\pi_{1}(\bar{B})$ and $i_{*}$ is surjective, one gets that $\rho_{F}$ has also finite image. It implies that $F_{\bar{B}}^{1,0}$ becomes trivial after a suitable finite étale base change. From this together with Deligne's global invariant cycle theorem (cf. [10, §4.1]) or Fujita's decomposition theorem (cf. [19], Theorem 3.1]), it follows that $\operatorname{rank} F_{\bar{B}}^{1,0}=q_{\bar{f}}$ after passing a finite étale base change.

\section{EXAmples AND MiscellaneOUs RESUlts}

In this section, we construct some Shimura curves contained generically in the Torelli locus in the low genus case, and prove miscellaneous related results.

Example 8.1. We construct a Shimura curve contained generically in the Torelli locus of hyperelliptic curves of genus $g=3$.

Let $C, H_{x_{0}} \subseteq X_{0}=\mathbb{P}^{1} \times \mathbb{P}^{1}$ be defined respectively by

$$
1+(4 t-2) x^{2}+x^{4}=0, \quad \text { and } \quad x=x_{0},
$$

where $t$ and $x$ are the coordinates of the first and second factor of $X_{0}$ respectively. The projection of $C$ to the first factor $\mathbb{P}^{1}$ of $X_{0}$ branches exactly over three points, i.e., $\{0,1, \infty\}$. Locally, it looks like the following.
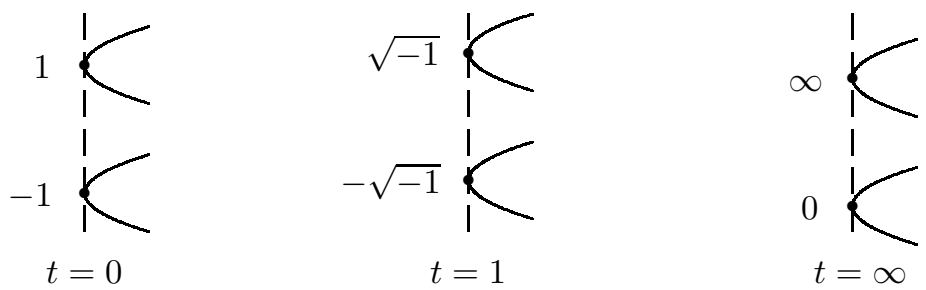

Let $\varphi: \mathbb{P}^{1} \rightarrow \mathbb{P}^{1}$ be the cyclic cover of degree 4 defined by $t=\left(t^{\prime}\right)^{4}$, totally ramified over $\{0, \infty\}$. Let $X_{1}$ be the normalization of the fibre-product $X_{0} \times_{\mathbb{P}^{1}} \mathbb{P}^{1}$ and $R$ the inverse image of

$$
C \cup H_{1} \cup H_{-1} \cup H_{0} \cup H_{\infty} .
$$

Then $R$ is a double divisor, i.e., we can construct a double cover $S_{1} \rightarrow X_{1}$ branched exactly over $R$. Let $S^{\prime} \rightarrow X_{r}$ be the canonical resolution, and $\bar{f}: \bar{S} \rightarrow \mathbb{P}^{1}$ the relatively minimal smooth model as follows. 


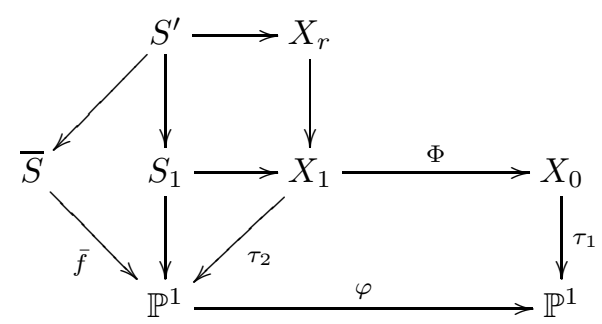

By the theory of double covers (cf. [4, $\S$ III.22]), it is not difficult to show that $\bar{f}: \bar{S} \rightarrow \mathbb{P}^{1}$ is a semi-stable hyperelliptic family of genus $g=3$. In fact, there are exactly 6 singular fibres in the family $\bar{f}$, i.e., those fibres $\Upsilon$ over $\Delta:=\varphi^{-1}(0 \cup 1 \cup \infty)$. More precisely, for any fibre $F$ over $\varphi^{-1}(1), F$ is an irreducible singular elliptic curve with exactly two nodes, hence $\xi_{0}(F)=2$ and $\delta_{1}(F)=\xi_{1}(F)=0$; for any fibre $F$ over $\varphi^{-1}(0 \cup \infty), F$ is a chain of three smooth elliptic curves, hence $\delta_{1}(F)=2$ and $\xi_{0}(F)=\xi_{1}(F)=0$. So

$$
\xi_{0}(\Upsilon)=8, \quad \delta_{1}(\Upsilon)=4, \quad \text { and } \quad \xi_{1}(\Upsilon)=0
$$

Therefore by (6-1),

$$
\operatorname{deg} \bar{f}_{*} \omega_{\bar{S} / \mathbb{P}^{1}}=2 .
$$

By definition, those fibres over $\Delta_{c t}:=\varphi^{-1}(0 \cup \infty)$ have compact Jacobian, while those over $\Delta_{n c}:=\varphi^{-1}(1)$ have non-compact Jacobian. Hence the Jacobian of $\bar{f}$ admits exactly $\left|\Delta_{n c}\right|=4$ singular fibres over $\mathbb{P}^{1}$. By [69, $\left.\S 7\right]$, the Higgs field of any semi-stable family of abelian varieties over $\mathbb{P}^{1}$ with exactly 4 singular fibres must be maximal. Hence the base $\mathbb{P}^{1} \backslash \Delta_{n c}$ (more precisely, the image of $\mathbb{P}^{1} \backslash \Delta_{n c}$ in $\mathcal{A}_{3}$ ) is a totally geodesic curve by Corollary 3.5 and Theorem 1.3 .

It remains to show that $\mathbb{P}^{1} \backslash \Delta_{n c}$ is in fact a Shimura curve. We present here two ways.

(i). Let

$$
\left(\bar{f}_{*} \omega_{\bar{S} / \mathbb{P}^{1}} \oplus R^{1} \bar{f}_{*} \mathcal{O}_{\bar{S}}, \theta_{\mathbb{P}^{1}}\right)=\left(A_{\mathbb{P}^{1}}^{1,0} \oplus A_{\mathbb{P}^{1}}^{0,1},\left.\theta_{\mathbb{P}^{1}}\right|_{A_{\mathbb{P}^{1}}^{1,0}}\right) \oplus\left(F_{\mathbb{P}^{1}}^{1,0} \oplus F_{\mathbb{P}^{1}}^{0,1}, 0\right)
$$

be the decomposition of the associated logarithmic Higgs bundle as in (3-3). Since the base is $\mathbb{P}^{1}$, one has $q_{\bar{f}}=\operatorname{rank} F_{\mathbb{P}^{1}}^{1,0}$. Hence by (3-5),

$$
2=\operatorname{deg} \bar{f}_{*} \omega_{\bar{S} / \mathbb{P}^{1}}=\operatorname{deg} A_{\mathbb{P}^{1}}^{1,0}=\frac{g-q_{\bar{f}}}{2} \cdot \operatorname{deg}\left(\Omega_{\mathbb{P}^{1}}^{1}\left(\log \Delta_{n c}\right)\right)=3-q_{\bar{f}}, \Longrightarrow q_{\bar{f}}=1 .
$$

Since the base $\mathbb{P}^{1}$ is simply connected, by [69, Theorem 0.2$]$, the relative Jacobian of $\bar{f}$ is isogenous over $\mathbb{P}^{1}$ to a product

$$
E \times_{\mathbb{P}^{1}} \mathcal{E} \times_{\mathbb{P}^{1}} \mathcal{E}
$$

where $E$ is a constant elliptic curve, and $\mathcal{E} \rightarrow \mathbb{P}^{1}$ is a family of semi-stable elliptic curves with maximal Higgs field. To show that $\mathbb{P}^{1} \backslash \Delta_{n c}$ is a Shimura curve, it suffices to prove that the constant part $E$ has complex multiplication.

It is not difficult to see that our family is actually defined by

$$
y^{2}=\left(1+\left(4\left(t^{\prime}\right)^{4}-2\right) x^{2}+x^{4}\right) \cdot\left(x^{2}-1\right) \cdot x .
$$

Let $E_{0}$ be a constant elliptic curve defined by $u^{4}=v \cdot(v+1)^{2}$. Then it is clear that $E_{0}$ has complex multiplication by $\mathbb{Z}[\sqrt{-1}]$. Define a morphism from the family $\bar{f}$ to the constant family $E_{0}$ by

$$
(u, v)=\psi(x, y)=\left(\frac{\sqrt{2} \cdot t^{\prime} y}{\left(x^{2}-1\right)^{2}}, \frac{4\left(t^{\prime}\right)^{4} x^{2}}{\left(x^{2}-1\right)^{2}}\right)
$$


It can be checked easily that $\psi$ is well-defined. Hence the Jacobian of $\bar{f}$ contains a constant part $E_{0}$. Note that the constant part $E$ in the decomposition (8-1) is unique up to isogenous, and the property with complex multiplication is invariant under isogenous. Therefore, the constant part $E \sim E_{0}$ has complex multiplication, and so $\mathbb{P}^{1} \backslash \Delta_{n c}$ is a Shimura curve.

(ii). We prove that $\mathbb{P}^{1} \backslash \Delta_{n c}$ is a Shimura curve by showing that our family $\bar{f}$ is actually isomorphic to a known special family constructed by Moonen and Oort [8]. Let

$$
u=\frac{1+x^{2}}{1-x^{2}}, \quad v=\frac{2 y}{\left(1-x^{2}\right)^{2}}, \quad w=\left(\frac{1+x^{2}}{1-x^{2}}\right)^{2} .
$$

Then by virtue of (8-2), we see that our family is isomorphic to

$$
\mathcal{U}_{t^{\prime}}:\left\{\begin{array}{l}
u^{2}=w \\
v^{4}=\left(2\left(t^{\prime}\right)^{4} w-2\left(\left(t^{\prime}\right)^{4}-1\right)\right)^{2} \cdot(w-1) .
\end{array}\right.
$$

Such a family can be viewed as a family of abelian covers of $\mathbb{P}^{1}$ branched exactly over 4 points with Galois group $\mathbb{Z}_{2} \times \mathbb{Z}_{4}$ and local monodromy of the branched points being $((1,0),(1,1),(0,1),(0,2))$. And it is just the family $(22)$ given in [48, $\S 6$, TABLE 2], which is special.

We remark that by 48 , we do not know whether the corresponding Shimura curve is complete or not (i.e., whether $\Delta_{n c}=\emptyset$ or not). Our concrete description shows that such a Shimura curve is a non-complete rational Shimura curve.

Example 8.2. We construct a Shimura curve with strictly maximal Higgs field contained generically in the Torelli locus of hyperelliptic curves of genus $g=4$.

The construction is similar to Example 8.1

Let $C, H_{x_{0}}$ and $X_{0}$ be the same as those in Example 8.1. Let $\varphi: \bar{B} \rightarrow \mathbb{P}^{1}$ be a cover of degree 8 , ramified uniformly over $\{0,1, \infty\}$ with ramification indices equal to 4 . It is easy to see that such a cover exists, and

$$
g(\bar{B})=2, \quad|\Delta|=6, \quad \text { where } \Delta=\varphi^{-1}(0 \cup 1 \cup \infty) .
$$

Let $X_{1}$ be the normalization of $X_{0} \times_{\mathbb{P}^{1}} \bar{B}$ and $R$ the inverse image of

$$
C \cup H_{1} \cup H_{-1} \cup H_{\sqrt{-1}} \cup H_{-\sqrt{-1}} \cup H_{0} \cup H_{\infty} \text {. }
$$

Then $R$ is a double divisor, i.e., we can construct a double cover $S_{1} \rightarrow X_{1}$ branched exactly over $R$. Let $\bar{f}: \bar{S} \rightarrow \bar{B}$ the relatively minimal smooth model as follows.

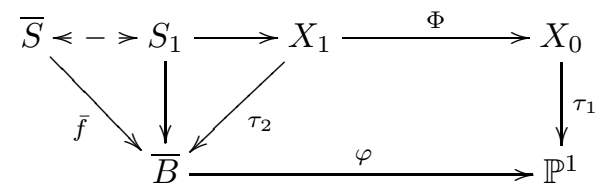

By [4, $\S$ III.22], one can show that $\bar{f}: \bar{S} \rightarrow \bar{B}$ is a semi-stable hyperelliptic family of genus $g=4$ with 6 singular fibres, i.e., those fibres $\Upsilon$ over $\Delta$. More precisely, for any fibre $F \in \Upsilon, F$ consists of two smooth elliptic curves $D_{1}, D_{2}$, and a smooth closed curve $\widetilde{D}$ of genus 2 , such that $D_{1}$ does not intersect $D_{2}$, and $\widetilde{D}$ intersects each $D_{i}$ transversely in one point for $i=1,2$. Hence

$$
\delta_{1}(F)=2, \quad \text { and } \quad \delta_{2}(F)=\xi_{0}(F)=\xi_{1}(F)=0, \quad \forall F \in \Upsilon .
$$

Since $|\Delta|=6$, by (2-2), (6-1) and (2-5) one gets

$$
\delta_{\bar{f}}=12, \quad \operatorname{deg} \bar{f}_{*} \omega_{\bar{S} / \bar{B}}=4, \quad \omega_{\bar{S} / \bar{B}}^{2}=36 .
$$

By definition, any singular fibre of $\bar{f}$ has a compact Jacobian, so the Jacobian of $\bar{f}$ is a smooth family of abelian varieties of dimension 4 . Let $A \bar{B}_{B}^{1,0} \subseteq \bar{f}_{*} \omega_{\bar{S}} / \bar{B}$ be the 
ample part in the decomposition (3-3). Then according to the Arakelov inequality, we have

$$
4=\operatorname{deg} \bar{f}_{*} \omega_{\bar{S} / \bar{B}} \leq \frac{\operatorname{rank} A_{\bar{B}}^{1,0}}{2} \cdot \operatorname{deg} \Omega_{\bar{B}}=\operatorname{rank} A_{\bar{B}}^{1,0} \leq \operatorname{rank} \bar{f}_{*} \omega_{\bar{S} / \bar{B}}=g=4 .
$$

Hence the Jacobian of $\bar{f}$ reaches the Arakelov bound with $\operatorname{rank} A^{1,0}=g$, i.e., the Higgs field associated to $\bar{f}$ is strictly maximal. Therefore $B=\bar{B}$ (more precisely, the image of $\bar{B}$ in $\mathcal{A}_{4}$ ) is a Shimura curve of type II by Corollary 3.5 and Theorem 1.3 since $\Delta_{n c}=\emptyset$.

We remark that in this example,

$$
c_{1}^{2}\left(\Omega \frac{1}{S}(\log D)\right)=3 c_{2}\left(\Omega \frac{1}{S}(\log D)\right)=72,
$$

where $D$ is the union of those 12 smooth disjoint elliptic curves contained in $\Upsilon$. Hence $\bar{S} \backslash D$ is a ball quotient by 30 or 43 .

Example 8.3. We construct a Shimura curve of type I contained generically in the Torelli locus $\mathcal{T}_{3}$, which can not be represented by a family $\bar{f}: \bar{S} \rightarrow \bar{B}$ of semi-stable curves of genus $g=3$ with strictly maximal Higgs field.

In Section 2.2, we have constructed Shimura curves of type I in $\mathcal{A}_{g}$ for each $g$. Since $\mathcal{T}_{3}=\mathcal{A}_{3}$, there is Shimura curve of type I contained in $\mathcal{T}_{3}$. Note that any Hecke translate of a Shimura curve of type I is still a Shimura curve of type I, and all the Hecke translates of such a curve are dense in $\mathcal{A}_{g}$. Therefore there must be a Shimura curve $C$ of type I contained generically in $\mathcal{T}_{3}$, and moreover we may find such a curve $C$ which is not contained in the Torelli locus of hyperelliptic curves. This implies that the family $\bar{f}$ of semi-stable curves representing $C$ by the Torelli morphism is non-hyperelliptic. Hence by Theorem 8.5 blow, $\bar{f}$ cannot have strictly maximal Higgs field.

The next proposition can be viewed as a byproduct of the proof of Theorem 4.5 .

Proposition 8.4. Let $\bar{f}: \bar{S} \rightarrow \bar{B}$ be a non-hyperelliptic family of semi-stable curves. Assume that $\bar{f}_{*} \omega_{\bar{S}} / \bar{B}$ is semi-stable. Then

$$
\omega \frac{2}{S} / \bar{B} \geq \frac{5 g-6}{g} \operatorname{deg} \bar{f}_{*} \omega_{\bar{S} / \bar{B}}+\sum_{p \in \Delta_{c t}}\left(3 l_{h}\left(F_{p}\right)+2 l_{1}\left(F_{p}\right)-3\right) .
$$

Proof. The proof is similar to that of Theorem 4.5. We use the same notations. The assumption that $\bar{f}_{*} \omega_{\bar{S}} / \bar{B}$ is semi-stable ensures that (6-21) is still valid. Note that (6-25) and (6-28) are true for any non-hyperelliptic semi-stable family. Hence (8-3) follows.

Theorem 8.5. Let $\bar{f}: \bar{S} \rightarrow \bar{B}$ be a non-isotrivial family of non-hyperelliptic semistable curves of genus $g \geq 3$. Then $\bar{f}$ cannot have strictly maximal Higgs field, i.e., we have the following strict Arakelov inequality

$$
\operatorname{deg} \bar{f}_{*} \omega_{\bar{S} / \bar{B}}<\frac{g}{2} \cdot \operatorname{deg} \Omega \frac{1}{B}\left(\log \Delta_{n c}\right) .
$$

Proof. This is an improvement of Theorem 4.6. It suffices to consider the cases $g=4$ or 3 according to Theorem 4.6 .

Consider first the case $g=4$. We argue by contradiction. Suppose (8-4) does not hold. Then by (4-8), one has

$$
\operatorname{deg} \bar{f}_{*} \omega_{\bar{S} / \bar{B}}=\frac{g}{2} \cdot \operatorname{deg} \Omega \frac{1}{B}\left(\log \Delta_{n c}\right)=2 \operatorname{deg} \Omega \frac{1}{B}\left(\log \Delta_{n c}\right) .
$$

It follows that the associated Higgs field $\theta_{\bar{B}}$ is strictly maximal, and both (4-1) and (4-2) are equalities. By Remarks [5.6(i), one has $\Delta_{n c}=\emptyset$ and $\Delta_{c t}=\Delta_{c t, u b}$, where 
$\Delta_{c t, u b}$ is defined in Theorem 5.1. Hence by (5-1) together with (8-5), one gets

$$
\omega_{\bar{S} / \bar{B}}^{2} \leq \frac{4(g-1)}{g} \cdot \operatorname{deg} \bar{f}_{*} \omega_{\bar{S} / \bar{B}}+\sum_{p \in \Delta_{c t}}\left(3 l_{h}\left(F_{p}\right)+2 l_{1}\left(F_{p}\right)-3\right) .
$$

As $\theta_{\bar{B}}$ is strictly maximal, $\bar{f}_{*} \omega_{\bar{S} / \bar{B}}=E_{\bar{B}}^{1,0}$ is poly-stable by [69, Proposition 1.2]. In particular, $\bar{f}_{*} \omega_{\bar{S} / \bar{B}}$ is semi-stable. Thus the condition of Proposition 8.4 is satisfied. It follows from (8-3) and (8-6) that $\operatorname{deg} f_{*} \omega_{\bar{S} / \bar{B}}=0$, which is impossible.

Now we consider the case $g=3$. Let $\overline{\mathcal{H}}_{3} \subseteq \overline{\mathcal{M}}_{3}$ be the locus of stable hyperelliptic curves, which is a divisor. Let $[H]$ be its divisor class. Let

$$
\varphi: \bar{B} \longrightarrow \overline{\mathcal{M}}_{3}, \quad p \mapsto\left[F_{p}\right]
$$

be the moduli map of $\bar{f}$, and $h=\operatorname{deg} \varphi^{*}([H])$. Then by [22, $\left.\S 3-\mathrm{H}\right]$, we have

$$
\begin{aligned}
\operatorname{deg} \bar{f}_{*} \omega_{\bar{S} / \bar{B}} & =\frac{1}{9} h+\frac{1}{9} \delta_{0}(\Upsilon)+\frac{1}{3} \delta_{1}(\Upsilon), \\
\omega \frac{2}{S} / \bar{B} & =\frac{4}{3} h+\frac{1}{3} \delta_{0}(\Upsilon)+3 \delta_{1}(\Upsilon) .
\end{aligned}
$$

Note that $\delta_{h}(\Upsilon)=0$ for $g=3$. It follows from (8-7), (8-8) and (4-1) that

$$
\begin{aligned}
\operatorname{deg} \bar{f}_{*} \omega_{\bar{S} / \bar{B}} & =\frac{3}{8} \omega \frac{2}{\bar{S} / \bar{B}}-\left(\frac{7}{18} h+\frac{1}{72} \delta_{0}(\Upsilon)+\frac{19}{24} \delta_{1}(\Upsilon)\right) \\
& \leq \frac{3}{2} \operatorname{deg} \Omega \frac{1}{B}\left(\log \Delta_{n c}\right)-\left(\frac{7}{18} h+\frac{1}{72} \delta_{0}(\Upsilon)+\frac{1}{24} \delta_{1}(\Upsilon)\right)
\end{aligned}
$$

Since $\bar{f}$ is non-isotrivial, one of $\left\{h, \delta_{0}(\Upsilon), \delta_{1}(\Upsilon)\right\}$ must be positive due to (8-7). Therefore, (8-4) follows immediately for $g=3$. The proof is complete.

Remarks 8.6. (i). Together with [20, 69], one can show that a family $\bar{f}$ of semi-stable genus- $g$ curves can have strictly maximal Higgs field only when $\bar{f}$ is hyperelliptic and $g=2$ or 4 . Indeed, by Theorems $\mathrm{D}$ and 8.5 . It suffices to exclude the case when $\bar{f}$ is hyperelliptic of genus $g=3$ with strictly maximal Higgs field. It this were the case, it is proven in [20] that $\Delta_{n c} \neq \emptyset$; by [69, Theorem 0.5], it follows that the relative Jacobian of $\bar{f}$ is isogenous a smooth family of abelian varieties over a Shimura curve of Mumford type up to a finite étale base change, which implies particularly that $g$ is even (cf Section 2.2), which is absurd.

(ii). We refer to [65, §3] for such examples of $g=2$ and Example 8.2 for an example of $g=4$.

\section{APPENDIX: INVOLUTIONS ON THE UNIVERSAL FAMILY OF CURVES}

The purpose of the appendix is to prove the existence of involutions on the universal family of curves. This is supposed to be a known result, and we include it for readers' convenience.

Recall that there is a universe family of stable curves over the partial compactification $\mathcal{M}_{g}^{c t}=\mathcal{M}_{g,[n]}^{c t}$ of the moduli space of smooth projective genus- $g$ curves with level- $n$ structure by adding those stable curves with compact Jacobians as in (3-1).

Lemma A.1. There exists an involution $\sigma_{g}\left(\right.$ resp. $\left.\tau_{g}\right)$ on $\mathcal{S}_{g}\left(\right.$ resp. $\left.\mathcal{M}_{g}^{c t}\right)$ such that the following diagram commutes.

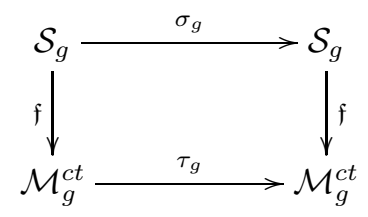


Moreover, the fixed locus of $\tau_{g}$ is exactly the hyperelliptic locus $\mathcal{H}_{g}^{\text {ct }} \subseteq \mathcal{M}_{g}^{c t}$, and for $p \in \mathcal{H}_{g}^{c t},\left.\sigma_{g}\right|_{C_{p}}: C_{p} \rightarrow C_{p}$ is the hyperelliptic involution of $C_{p}$, where $C_{p} \subseteq \mathcal{S}_{g}$ is the hyperelliptic curve over $p$.

Proof. According to [56], there exists an involution $\tau_{g}$ on $\mathcal{M}_{g}$. But it is easy to see that it can be extended to $\mathcal{M}_{g}^{c t}$, which is defined by

$$
\tau_{g}([C, \alpha])=[C,-\alpha],
$$

where $C$ is a genus- $g$ stable curve of compact type and $\alpha$ is a level- $n$ structure of $C$. Note that for a hyperelliptic curve $C$, the hyperelliptic involution gives an isomorphism between $[C, \alpha]$ and $[C,-\alpha]$. It follows that (cf. [56]) the fixed point of the involution $\tau_{g}$ is exactly the hyperelliptic locus $\mathcal{H}_{g}^{c t} \subseteq \mathcal{M}_{g}^{c t}$.

Let $\mathcal{M}_{g, 1}=\mathcal{M}_{g,[n], 1}$ (resp. $\mathcal{M}_{g, 1}^{c t}=\mathcal{M}_{g,[n], 1}^{c t}$ ) be the moduli space of genus- $g$ smooth curves (resp. stable curves of compact type) with level- $n$ structure and one marked point, and $\operatorname{Fog}_{g}^{\mathrm{o}}: \mathcal{M}_{g, 1} \rightarrow \mathcal{M}_{g}$ (resp. Fog $: \mathcal{M}_{g, 1}^{c t} \rightarrow \mathcal{M}_{g}^{c t}$ ) the natural map by forgetting the marked point. It is easy to see that $\operatorname{Fog}_{g}^{\mathrm{o}}$ (resp. Fog $_{g}$ ) factors through $\mathcal{S}_{g}^{\circ}=\mathfrak{f}^{-1}\left(\mathcal{M}_{g}\right)\left(\right.$ resp. $\left.\mathcal{S}_{g}\right)$

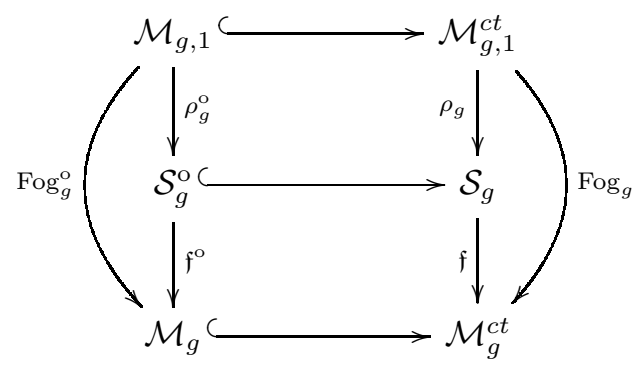

Note that $\rho_{g}^{\mathrm{o}}$ is actually an isomorphism; indeed, for any $p \in \mathcal{M}_{g}$, the fibres over $p$ in both $\mathcal{S}_{g}^{\circ}$ and $\mathcal{M}_{g, 1}$ is isomorphic to $C_{p}$, where $C_{p}$ is the smooth curve corresponding to $p$. However, it is not the case for $\rho_{g}$. By the definition of $\rho_{g}$, it just maps a stable curve with one marked point to the curve. But a stable curve with one marked point may become a non-stable curve when forgetting the marked point. Hence if $p \in \mathcal{M}_{g}^{c t} \backslash \mathcal{M}_{g}$, then $C_{p, 1} \rightarrow C_{p}$ is just the contraction of non-stable components contained in $C_{p, 1}$, where $C_{p, 1}$ (resp. $C_{p}$ ) be the fibre over $p$ in $\mathcal{M}_{g, 1}$ (resp. $\mathcal{S}_{g}^{o}$ ).

We define an involution $\sigma_{g, 1}$ on $\mathcal{M}_{g, 1}^{c t}$ by

$$
\sigma_{g, 1}([C, \alpha, x])=[C,-\alpha, x],
$$

where $C$ is a genus- $g$ stable curve of compact type, $\alpha$ is a level- $n$ structure of $C$, and $x \in C$ is a marked point. Then it is clear that

$$
\operatorname{Fog}_{g} \circ \sigma_{g, 1}=\tau_{g} \circ \operatorname{Fog}_{g} .
$$

Define an involution $\sigma_{g}$ on $\mathcal{S}_{g}$ by

$$
\sigma_{g}(x)=\rho_{g} \circ \sigma_{g, 1} \circ \rho_{g}^{-1}(x) .
$$

According to the description of $\rho_{g}$ above, $\sigma_{g}$ is well-defined. And the diagram in the lemma commutes by (A-2).

If $p \in \mathcal{H}_{g}^{c t}$, then the fibre $C_{p, 1}$ over $p$ in $\mathcal{M}_{g, 1}^{c t}$ is a stable hyperelliptic curve with one marked point. The hyperelliptic involution $\iota$ induces an isomorphism

$$
\left[C_{p, 1}, \alpha, x\right] \stackrel{\cong}{\longrightarrow}\left[C_{p, 1},-\alpha, \iota(x)\right] .
$$

Hence

$$
\sigma_{g, 1}\left(\left[C_{p, 1}, \alpha, x\right]\right)=\left[C_{p, 1},-\alpha, x\right]=\left[C_{p, 1}, \alpha, \iota(x)\right]
$$


It implies that $\left.\sigma_{g, 1}\right|_{C_{p .1}}: C_{p, 1} \rightarrow C_{p, 1}$ is just the hyperelliptic involution of $C_{p, 1}$. Hence $\left.\sigma_{g}\right|_{C_{p}}: C_{p} \rightarrow C_{p}$ is the hyperelliptic involution of $C_{p}$.

Acknowledgements. We would like to thank Chris Peters, Guitang Lan and Jinxing $\mathrm{Xu}$ for discussions on the topic related to global invariant cycles with locally constant coefficients. Especially, the proof of Lemma 7.1 comes from a discussion with Chris Peters. We would also like to thank Shengli Tan and Hao Sun for discussing with us on Miyaoka-Yau's inequality and the slope inequality, and Alessandro Ghigi and Stefan Müller-Stach for their interests. We thank Ke Chen for discussing with us on Shimura varieties and the formulation of the paper. We are grateful to Yanhong Yang for her interests, careful reading and valuable suggestions..

\section{REFERENCES}

[1] M. Bainbridge and M. Möller, The locus of real multiplication and the Schottky locus, J. Reine Angew. Math. 686 (2014), 167-186.

[2] W. Baily and A. Borel, Compactification of arithmetic quotients of bounded symmetric domains, Annals of mathematics 84(2) (1966), 442-528.

[3] M. Á. Barja, On the slope and geography of fibred surfaces and threefolds, Ph.D. Thesis of Universitat de Barcelona. (1998).

[4] W. P. Barth, K. Hulek, C. A. M. Peters and A. Van de Ven, Compact Complex Surfaces, (Second Enlarged Edition), A Series of Modern Surveys in Mathematics, Volume 4, SpringerVerlag, (2004).

[5] C.-L. Chai and F. Oort, Abelian varieties isogenous to a Jacobian, Ann. of Math. (2) 176(1) (2012), 589-635.

[6] K. Chen, On special subvarieties of Kuga varieties, Mathematische Zeit. 274 (2013), 821-839.

[7] R. F. Coleman, Torsion points on curves, Galois representations and arithmetic algebraic geometry (Kyoto, 1985 /Tokyo, 1986), 235-247, Adv. Stud. Pure Math., 12, North-Holland, Amsterdam, 1987.

[8] E. Colombo, P. Frediani and A. Ghigi, On totally geodesic submanifolds in the Jacobian locus, arXiv:1309.1022

[9] M. Cornalba and J. Harris, Divisor classes associated to families of stable varieties, with applications to the moduli space of curves, Ann. Sc. Ec. Norm. Sup., 21 (1988), 455-475.

[10] P. Deligne, Théorie de Hodge II, Inst. Hautes études Sci. Publ. Math., 40 (1971), 5-58.

[11] — Variétés de Shimura: interprtation modulaire, et techniques de construction de modles canoniques, (French), Automorphic forms, representations and L-functions (Proc. Sympos. Pure Math., Oregon State Univ., Corvallis, Ore., 1977), Part 2, pp. 247-289, Proc. Sympos. Pure Math., XXXIII, Amer. Math. Soc., Providence, R.I., 1979.

[12] - Hodge cycles on abelian varities (Notes by J. S. Milne), in: Hodge cycles, motives, and Shimura varieties. Lecture Notes in Mathematics, 900. Springer-Verlag, Berlin-New York, 1982, 9-100.

[13] P. Deligne and D. Mumford, The irreducibility of the space of curves of given genus, Inst. Hautes études Sci. Publ. Math., 36 (1969), 75-109.

[14] B. Dwork and A. Ogus, Canonical liftings of Jacobians, Compositio Math. 58 (1986), 111131.

[15] T. Ekedahl and J.-P. Serre, Exemples de courbes algébriques à jacobienne complétement décomposable, C. R. Acad. Sci. Paris Sér. I Math. 317 (1993), 509-513.

[16] G. Faltings, Arakelov's theorem for abelian varieties, Invent. math. 73 (1983), 337-348.

[17] B. Farb and H. Masur, Superrigidity and mapping class groups, Topology, 37(6) (1998), 1169-1176.

[18] P. Frediani, A. Ghigi and M. Penegini, Shimura varieties in the Torelli locus via Galois coverings http://arxiv.org/abs/1402.0973v2

[19] T. Fujita, On Kähler fiber spaces over curves, J. Math. Soc. Japan 30(4) (1978), 779-794.

[20] S. Grushevsky and M. Möller, Shimura curves within the locus of hyperelliptic Jacobians in genus three, http://arxiv.org/abs/1308.5155

[21] R. Hain, Locally symmetric families of curves and Jacobians, Moduli of curves and abelian varieties, 91-108, Aspects Math., E33, Vieweg, (1999).

[22] J. Harris and I. Morrison, Moduli of curves, Graduate Texts in Mathematics, 187. SpringerVerlag, New York, (1998). 
[23] J. Harris and D. Mumford, On the Kodaira dimension of the moduli space of curves, With an appendix by William Fulton. Invent. Math. 67(1) (1982), 23-88.

[24] R. Hartshorne, Algebraic Geometry, GTM 52, Springer-Verlag, (1997).

[25] H. Hida, p-adic automorphic forms on Shimura varieties, Springer-Verlag 2004.

[26] J. de Jong and R. Noot, Jacobians with complex multiplication, Arithmetic algebraic geometry (Texel, 1989), 177-192, Progr. Math., 89, Birkhäuser Boston, Boston, MA, (1991).

[27] J. de Jong and S.-W. Zhang, Generic Abelian Varieties with Real Multiplication are not Jacobians, Diophantine geometry, CRM Series, 4 (2007), 165-172.

[28] S. I. Khashin, The irregularity of double surfaces, Mathematical notes of the Academy of Sciences of the USSR, 33(3) (1983), 233-235.

[29] B. Klingler and A. Yafaev, The André-Oort conjecture, to appear in Ann. of Math.

[30] R. Kobayashi, Einstein-Kaehler metrics on open algebraic surfaces of general type, Tohoku Math. J. 37(2) (1985), no. 1, 43-77.

[31] J. Kollár, Subadditivity of the Kodaira dimension: fibers of general type, Algebraic Geometry, Sendai, 1985. Advanced Studies in Pure Mathematics 10 (1987), 361-398.

[32] S. S. Kudla, Algebraic cycles on Shimura varieties of orthogonal type, Duke Math. J. 86 (1997), no. 1, 39-78.

[33] S. S. Kudla and M. Rapoport, Special cycles on unitary Shimura varieties II: global theory arXiv:0912.3758, Accepted for publication in Crelle.

[34] S. Kukulies, On Shimura curves in the Schottky locus, J. Algebraic Geom. 19(2) (2010), $371-397$.

[35] R. Lazarsfeld, Positivity in algebraic geometry. II. Positivity for vector bundles, and multiplier ideals, Springer-Verlag, Berlin, 2004.

[36] K.-F. Liu, Geometric height inequalities, Math. Res. Lett. 3(5) (1996), 693-702.

[37] J. Lu, S.-L. Tan, F. Yu and K. Zuo, A new inequality on the Hodge number $h^{1,1}$ of algebraic surfaces, Math. Z. 276 (2014), no. 1-2, 543-555.

[38] X., Lu, K., Zuo, On Shimura curves in the Torelli locus of curves, http://arxiv.org/abs/1311.5858

[39] J. Milne, Canonical Models of (Mixed) Shimura Varieties and Automorphic Vector Bundles, in: Automorphic Forms, Shimura Varieties, and L-functions, (Proceedings of a Conference held at the University of Michigan, Ann Arbor, July 6-16, 1988), pp. 283-414.

[40] J. S., Milne, Introduction to Shimura varieties, Harmonic analysis, the trace formula, and Shimura varieties, 265-378, Clay Math. Proc., 4, Amer. Math. Soc., Providence, RI, 2005

[41] Y. Miyaoka, The maximal number of quotient singularities on surfaces with given numerical invariants, Math. Ann. 268 (1984), 159-171.

[42] A. Mohajer and K. Zuo, On Shimura subvarieties generated by families of abelian covers of $\mathbb{P}^{1}$, http://arxiv.org/abs/1402.1900

[43] N. Mok, Projective algebraicity of minimal compactifications of complex-hyperbolic space forms of finite volume, Perspectives in analysis, geometry, and topology, 331-354, Progr. Math., 296, Birkhäuser/Springer, New York, 2012.

[44] M. Möller, Shimura and Teichmüller curves, J. Mod. Dyn. 5(1) (2011), 1-32.

[45] M. Möller, E. Viehweg and K. Zuo, Stability of Hodge bundles and a numerical characterization of Shimura varieties, J. Differential Geom. 92(1) (2012), 71-151.

[46] B. Moonen, Linearity properties of Shimura varieties, I, J. Algebraic Geom. 7(3) (1998), $539-567$.

[47] - Special subvarieties arising from families of cyclic covers of the projective line, Doc. Math. 15 (2010), 793-819.

[48] B. Moonen and F. Oort, The Torelli locus and special subvarieties, Handbook of moduli. Vol. II, 549-594, Adv. Lect. Math. (ALM), 25, Int. Press, Somerville, MA, 2013.

[49] A. Moriwaki, Relative Bogomolov's inequality and the cone of positive divisors on the moduli space of stable curves, J. Amer. Math. Soc. 11(3) (1998), 569-600.

[50] D. Mumford, Geometric invariant theory, Ergebnisse der Mathematik und ihrer Grenzgebiete, Neue Folge, Band 34 Springer-Verlag, Berlin-New York 1965.

[51] — - A Note of Shimura's Paper "Discontinuous Groups and Abelian Varieties", Math. Ann. 181 (1969), 345-351.

[52] — Abelian varieties. With appendices by C. P. Ramanujam and Yuri Manin. Corrected reprint of the second (1974) edition. Tata Institute of Fundamental Research Studies in Mathematics, 5. Published for the Tata Institute of Fundamental Research, Bombay; by Hindustan Book Agency, New Delhi, 2008.

[53] - Hirzebruch's proportionality theorem in the noncompact case, Invent. Math. 42 (1977), 239-272. 
[54] R. Noot, Correspondances de Hecke, action de Galois et la conjecture d'André-Oort (d'après Edixhoven et Yafaev), (French). Séminaire Bourbaki. Vol. 2004/2005. Astérisque No. 307 (2006), Exp. No. 942, vii, 165-197.

[55] F. Oort, Canonical liftings and dense sets of CM-points, in: Arithmetic geometry (Cortona, 1994), 228-234, Sympos. Math., XXXVII, Cambridge Univ. Press, Cambridge, (1997).

[56] F. Oort and J. Steenbrink, The local Torelli problem for algebraic curves, Journées de Géometrie Algébrique d'Angers, (1979), 157-204.

[57] C. Peters, Inequalities for semi-stable surface fibrations, and strictly maximal Higgs fields, http://arxiv.org/abs/1405.4531

[58] C. Peters and M. Saito, Lowest weights in cohomology of variations of Hodge structure, Nagoya Math. J. 206 (2012), 1-24.

[59] C. Peters and J. Steenbrink, Mixed Hodge structures, Ergebnisse der Mathematik und ihrer Grenzgebiete. 3. Folge. A Series of Modern Surveys in Mathematics, 52. Springer-Verlag, Berlin, 2008.

[60] H. Popp, Moduli theory and classification theory of algebraic varieties, Lecture Notes in Math. 620, Springer-Verlag 1977.

[61] J. C. Rhode, Cyclic coverings, Calabi-Yau manifolds and complex multiplication, Lecture Notes in Mathematics, 1975. Springer-Verlag, Berlin, 2009.

[62] F. Sakai, Semistable curves on algebraic surfaces and logarithmic pluricanonical maps, Math. Ann. 254(2) (1980), 89-120.

[63] T. Scanlon, A proof of the André-Oort conjecture via mathematical logic, after J. Pila, Séminaire Bourbaki, Exposé 1037, 2011

[64] S.-L. Tan, The minimal number of singular fibers of a semistable curve over $\mathbb{P}^{1}$, J. Algebraic Geom. 4(3) (1995), 591-596.

[65] Y. -P. Tu, Surfaces of Kodaira dimension zero with six semistable singular fibers over $\mathbb{P}^{1}$, Math. Z. 257(1) (2007), 1-5.

[66] E. Ullmo and A. Yafaev, Galois orbits and equidistribution: towards the André-Oort conjecture, preprint submitted, arXiv:1209.0934

[67] E. Viehweg, Arakelov inequalities, Surveys in differential geometry. Vol. XIII. Geometry, analysis, and algebraic geometry: forty years of the Journal of Differential Geometry, 245275, Surv. Differ. Geom., 13, Int. Press, Somerville, MA, 2009.

[68] E. Viehweg and K. Zuo, Families over curves with a strictly maximal Higgs field, Asian J. Math. 7(4) (2003), 575-598.

[69] — - A characterization of certain Shimura curves in the moduli stack of abelian varieties, J. Differential Geom. 66(2) (2004), 233-287.

[70] P. Vojta, Diophantine inequalities and Arakelov theory, in Lang, Introduction to Arakelov Theory, Springer-Verlag, (1988), 155-178.

[71] A. Yafaev, Galois orbits and equidistrubition: Manin-Mumford and André-Oort, Journal de la théorie de nombres de Bordeaux, 21(2), 493-502, 2009

[72] G. Xiao, Irregular families of hyperelliptic curves, Algebraic geometry and algebraic number theory (Tianjin, 1989-1990), 152-156, Nankai Ser. Pure Appl. Math. Theoret. Phys., 3, World Sci. Publ., River Edge, NJ, 1992.

Institut für Mathematik, Universität Mainz, Mainz, Germany, 55099

E-mail address: lvxinwillv@gmail.com

Institut für Mathematik, Universität Mainz, Mainz, Germany, 55099

E-mail address: zuok@uni-mainz.de 